SLAC-R-871

May 2007

\title{
Measurement of the Inclusive Branching Fractions $B\left(B^{\wedge}{ }_{-}\right.$to $\left.D^{\wedge+} P i^{\wedge_{-}} \mathbf{P i}^{\wedge_{-}}\right)$and $B\left(B^{\wedge-}\right.$ to $\left.D^{* \wedge+} \mathbf{P i}^{\wedge_{-}} \mathbf{P i}^{\wedge_{-}}\right)$
}

Vance O. Eschenburg

SLAC-R-871

Prepared for the Department of Energy

under contract number DE-AC02-76SF00515

Printed in the United States of America. Available from the National Technical Information Service, U.S. Department of Commerce, 5285 Port Royal Road, Springfield, VA 22161. 
This document, and the material and data contained therein, was developed under sponsorship of the United States Government. Neither the United States nor the Department of Energy, nor the Leland Stanford Junior University, nor their employees, nor their respective contractors, subcontractors, or their employees, makes an warranty, express or implied, or assumes any liability of responsibility for accuracy, completeness or usefulness of any information, apparatus, product or process disclosed, or represents that its use will not infringe privately owned rights. Mention of any product, its manufacturer, or suppliers shall not, nor is it intended to, imply approval, disapproval, or fitness of any particular use. A royalty-free, nonexclusive right to use and disseminate same of whatsoever, is expressly reserved to the United States and the University. 


\title{
Measurement of the Inclusive Branching Fractions $\mathcal{B}\left(B^{-} \rightarrow D^{+} \pi^{-} \pi^{-}\right)$and $\mathcal{B}\left(B^{-} \rightarrow D^{*+} \pi^{-} \pi^{-}\right)$
}

\author{
A Dissertation Presented for the \\ Doctor of Philosophy Degree \\ The University of Mississippi at Oxford
}

Vance Onno Eschenburg

May 2007 
Copyright (c) 2007 by Vance O. Eschenburg.

All rights reserved. 


\section{DEDICATION}

This work is dedicated to:

Zdravko Stipcevic

and

Nello McGowan

Many of my friends have passed on and cannot celebrate this joyous event with me. I wish you were here. 


\section{Acknowledgments}

I am eternally grateful to the many people who have helped and encouraged me during this great adventure. And I am particularly thankful to:

My dissertation advisor, Rob Kroeger, and the rest of my dissertation committee: Don Summers, Lucien Cremaldi, Luca Bombelli, and Gerard Buskes.

Johannes Bauer, Haiwen Zhao, Romulus Godang, David Sanders, and the rest of the University of Mississippi High Energy Physics group.

Iowa State University's BABAR research group: Jong Yi, Vitaly Eyges, Liaoyuan Dong, Soeren Prell, and Jim Cochran.

Candice and Robin McMinn, Kris and John Geering, Michael McPherson and 
the rest of my friends.

The Stanford Linear Accelerator Center, the BABAR Collaboration and the Physics Department of the University of Mississippi at Oxford.

Henry Bass and the National Center for Physical Acoustics.

The Ole Miss Physics Department's administrative assistants: Cherry Douglas, Mottsee Spurgeon, Mary Taylor, and Cindy Basket.

Donald Cole and the rest of the Graduate School at the University of Mississippi at Oxford.

And my Mom, Anna; my Dad, Vernon; and my brother, Jarrett.

Without any of you, none of this could have happened. Thank you. 


\begin{abstract}
The $D_{J}^{0}$ is a family of four orbitally excited mesons: $D_{2}^{*}(2460)^{0}, D_{1}(2420)^{0}$, $D_{1}\left(j=\frac{1}{2}\right)^{0}$, and $D_{0}^{*}\left(j=\frac{1}{2}\right)^{0}$. This dissertation presents the measurements of the inclusive branching fractions, $\mathcal{B}\left(B^{-} \rightarrow D^{*+} \pi^{-} \pi^{-}\right)$and $\mathcal{B}\left(B^{-} \rightarrow D^{+} \pi^{-} \pi^{-}\right)$. The $D_{J}^{0}$ provides an intermediate resonance for those two modes. The data used for this analysis consists of Runs $1-5$ with total integrated luminosity of $343.38 \mathrm{fb}^{-1}$, which is corresponding to 383.92 million $B \bar{B}$ pairs, provided by the BABAR detector at the PEP-II asymmetric $B$ Factory.
\end{abstract}

The values presented are:

$$
\begin{aligned}
\mathcal{B}\left(B^{-} \rightarrow D^{+} \pi^{-} \pi^{-}\right) & =(1.12 \pm 0.02 \pm 0.08) \times 10^{-3} \\
\mathcal{B}\left(B^{-} \rightarrow D^{*+} \pi^{-} \pi^{-}\right) & =(1.67 \pm 0.03 \pm 0.13) \times 10^{-3}
\end{aligned}
$$




\section{Contents}

1 Introduction 1

1.1 The $D_{J}^{0}$ Spectroscopy . . . . . . . . . . . . . . . . 1

1.2 PEP-II and BABAR .................. . . . 7

1.3 Procedure ....................... . . . . 9

2 BABAR Detector and PEP-II $\quad 11$

2.1 Introduction . . . . . . . . . . . . . . . . . . 11

2.2 The Asymmetry B-Factory, PEP-II . . . . . . . . . . . . . . . 14

2.3 Silicon Vertex Detector $(\mathrm{SVT})$. . . . . . . . . . . . 15

2.4 Drift Chamber $(\mathrm{DCH})$. . . . . . . . . . . . . 17

2.5 Detector of Internally Reflected Cerenkov Light (DIRC) . . . . 19

2.6 Electromagnetic Calorimeter (EMC) . . . . . . . . . . 23

2.7 Instrumented Flux Return (IFR) . . . . . . . . . . . . . 25

2.8 Vertexing and Fitting . . . . . . . . . . . . . . . 26 
2.9 Trigger System . . . . . . . . . . . . . . . . . . 27

3 Theoretical Background of $D_{J}^{0} \quad 28$

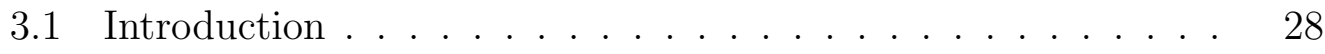

3.2 Heavy Quark Effective Theory _ . . . . . . . . . . 32

3.3 Introduction to the $D_{J}^{0} \quad \ldots \ldots \ldots \ldots \ldots \ldots$

$3.4 \quad D_{J}^{0}$ Allowed Decay Example $\ldots \ldots \ldots \ldots \ldots$

3.5 Narrow and Wide Resonances . . . . . . . . . . . . 36

4 Analysis $\quad 38$

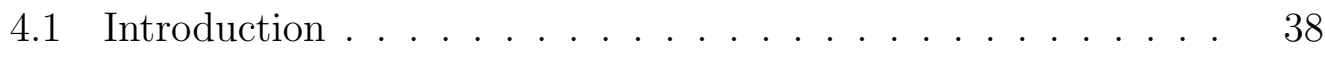

4.2 Data Acquisition . . . . . . . . . . . . . . . . . . . 39

4.3 Four Vectors - Particle Reconstruction _. . . . . . . . 40

4.4 Base Modes . . . . . . . . . . . . . . . . . . . . . . . 41

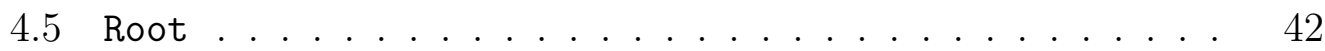

4.6 Available Data . . . . . . . . . . . . . . . . . . 42

4.7 Monte Carlo (MC) Samples . . . . . . . . . . . . . . . . 43

4.8 Event Reconstruction and Selection . . . . . . . . . . 45

4.8.1 Charged Track Selection . . . . . . . . . . . . 47

$4.8 .2 \quad \pi^{0}$ Candidates . . . . . . . . . . . . . . . . . 48

$4.8 .3 \quad D^{0}$ Reconstruction $\ldots \ldots \ldots \ldots \ldots$ 
4.8.4 $D^{*+}$ Reconstruction . . . . . . . . . . . . 49

4.8.5 $D^{+}$Reconstruction ............... 50

4.8.6 $\quad B^{ \pm}$Reconstruction . . . . . . . . . . . . . 50

4.9 Event Variables . . . . . . . . . . . . . . . . . . 51

4.9 .1 B Candidate $m_{E S} \ldots \ldots \ldots 52$

4.9 .2 Event Shape . . . . . . . . . . . . . 53

4.9.3 Thrust Axis Opening Angle . . . . . . . . . . . 54

4.9 .4 Dalitz Weight . . . . . . . . . . . . 55

4.10 Selection Optimization .............. 57

4.11 Measuring Inclusive Branching Fraction . . . . . . . . . . . . 58

4.11.1 Inclusive Measurement Calculation for $B^{-} \rightarrow D^{+} \pi^{-} \pi^{-} \quad 58$

4.11.2 Inclusive Measurement Calculation for $B^{-} \rightarrow D^{*+} \pi^{-} \pi^{-} \quad 59$

4.12 Uncertainties .................... 60

4.12.1 Statistical Uncertainty . . . . . . . . . . . 61

4.12 .2 Systematic Uncertainties . . . . . . . . . . . 61

4.12 .3 B-Counting . . . . . . . . . . . . . . 63

4.12.4 $D^{*+}, D^{0}, D^{+}$Branching Fraction Uncertainties $\ldots . .64$

4.12.5 Uncertainties on Efficiencies . . . . . . . . . . 65

4.12.6 MC Efficiency Differences . . . . . . . . . . 66

4.12 .7 Multiple Candidates . . . . . . . . . . . . 67 
4.12 .8 Tracking Efficiencies _. . . . . . . . . . . . . 67

4.12 .9 Particle Identification . . . . . . . . . . . . . . . 68

$4.12 .10 \pi^{0}$ Mass Resolution . . . . . . . . . . . . . . . 69

$\begin{array}{lll}5 & \text { Results, Summary and Conclusion } & 71\end{array}$

5.1 Results . . . . . . . . . . . . . . . . . . . 71

$5.2 \quad D^{0}$ and $D^{+}$Reconstructions $\ldots \ldots \ldots \ldots \ldots$

$5.3 \Delta\left(m\left(D^{*+}\right)-m\left(D^{0}\right)\right)$ Mass Difference . . . . . . . . 74

5.4 The Distributions of $m_{E S}$ and $\Delta E \ldots \ldots \ldots \ldots \ldots$

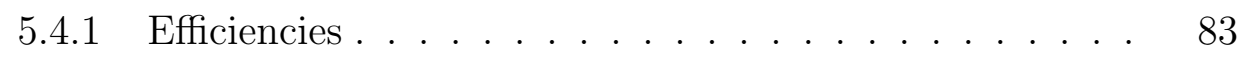

5.4.2 Peaking Background in the $\Delta E$ Distribution . . . . . 84

5.4 .3 Inclusive Results . . . . . . . . . . . . . . . . . 85

5.4.4 Discussion and Comparison _. . . . . . . . . 93

5.5 Results . . . . . . . . . . . . . . . . . . . . 95

5.6 Postscript: Hints of Future Directions . . . . . . . . . . 96

5.7 Closing . . . . . . . . . . . . . . . . . . . . . . . . 99 


\section{List of Tables}

1.1 Properties of the Six Known Quarks. . . . . . . . . . . . 2

1.2 Properties of $D_{J}^{0}$ spectroscopy including their mass, width and allowed transitions. .................. 5

1.3 Previous measurements of $\mathcal{B}\left(B^{-} \rightarrow D^{+} \pi^{-} \pi^{-}\right)$and $\mathcal{B}\left(B^{-} \rightarrow\right.$ $\left.D^{*+} \pi^{-} \pi^{-}\right) . \ldots \ldots \ldots \ldots \ldots$

1.4 Measurements and Calculation for R. . . . . . . . . . . . 6

1.5 Reconstructed Mode Chains for $B^{-} \rightarrow D^{+} \pi^{-} \pi^{-}$and $B^{-} \rightarrow$ $D^{*+} \pi^{-} \pi^{-} \ldots \ldots \ldots \ldots \ldots \ldots \ldots$

3.1 The four forces and their relative strengths. . . . . . . . . .

4.1 Decay modes used for the analysis. . . . . . . . . . . . . . . . 41

4.2 Overview of data and generic Monte Carlo samples used in the

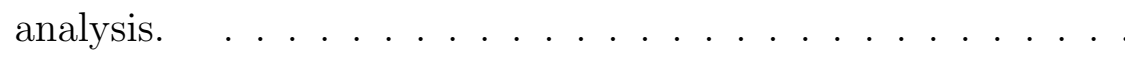

4.3 Table of non-generic Monte Carlo samples used in the analysis. 44 
4.4 Data collection dates . . . . . . . . . . . . . . 45

4.5 Table of cut variables' values. . . . . . . . . . . . . . 53

4.6 Branching fraction and uncertainties for $D^{0}, D^{+}$, and $D^{*+} \ldots \quad 64$

4.7 Submodes used for efficiency measurements. . . . . . . . . 65

4.8 MC Statistics Systematic Errors. . . . . . . . . . . . . 66

4.9 Efficiency differences. . . . . . . . . . . . . . . . . 67

4.10 Systematic uncertainty of the multiple candidates. . . . . . 67

4.11 Systematic Uncertainty due to Tracking. . . . . . . . . . . 68

4.12 Relative systematic uncertainties of $\mathcal{B}\left(B^{-} \rightarrow D^{*+} \pi^{-} \pi^{-}\right)$and $\mathcal{B}\left(B^{-} \rightarrow D^{+} \pi^{-} \pi^{-}\right)$with $D^{0} \rightarrow K^{-} \pi^{+}, D^{0} \rightarrow K^{-} \pi^{+} \pi^{0}, D^{0} \rightarrow$ $K^{-} \pi^{+} \pi^{-} \pi^{+}$, and $D^{+} \rightarrow K^{-} \pi^{+} \pi^{+} \ldots \ldots \ldots \ldots$

5.1 The $D^{0}$ fit results for data and generic Monte Carlo. The generated value of the mass for the generic and signal MC is set to be $1864.5 \mathrm{MeV} / c^{2} \ldots \ldots \ldots \ldots \ldots \ldots$

$5.2 \Delta\left(m\left(D^{*+}\right)-m\left(D^{0}\right)\right)$ measurements. . . . . . . . 75

5.3 The $\Delta E$ reconstruction efficiencies for signal Monte Carlo events in percentage. . . . . . . . . . . . .

5.4 The $D^{0}$ and $D^{+}$branching fractions of $D^{0}$ and $D^{+}$as given in the PDG $[80] \ldots \ldots \ldots \ldots \ldots \ldots$

5.5 BELLE numbers used for efficiency estimation [12]. . . . . . 84 
5.6 Estimates of peaking backgrounds from generic $B \bar{B}$ events to the fitted $\Delta E$ signal peaks for the selected $B^{-} \rightarrow D^{*+} \pi^{-} \pi^{-}$and $B^{-} \rightarrow D^{+} \pi^{-} \pi^{-}$samples. . . . . . . . . . . . . . 91

5.7 The $\Delta E$ fit results: $\Delta E$ mean and resolution, number of signal candidates, $Q^{2}$ optimization ratio in the signal region. . . . . .

5.8 Inclusive branching ratios for $B^{-} \rightarrow D^{*+} \pi^{-} \pi^{-}$for each $D^{0}$ decay mode. The errors are statistical only. . . . . . . . . . .

5.9 Comparison among the measurements. . . . . . . . . .

5.10 Calculated branching fractions after removing PHOTOS events in the signal MC. The errors are statistical only. . . . . . . . .

5.11 Calculated branching fractions after applying $m_{\mathrm{ES}}$-based best candidate selection. The errors are statistical only. . . . . . . .

5.12 Branching fractions used in the generation of simulated sample are compared to those measured by using the analysis method. 


\section{List of Figures}

1.1 Heavy Quark Effective Theory (HQET). . . . . . . . . . . 3

1.2 Spectroscopy of $D_{J}^{0}$. This figure shows the allowed $\pi$ transitions

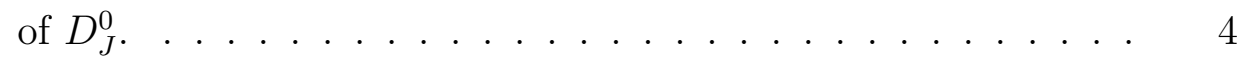

1.3 The BABAR detector. . . . . . . . . . . . . . . . . . 7

1.4 SLAC / PEP2. . . . . . . . . . . . . . . 8

1.5 Stanford Linear Accelerator Center (SLAC). . . . . . . . . . . 9

2.1 Opened view of BABAR. . . . . . . . . . . . . . . 13

2.2 PEP-II Delivered and BABAR Integrated Luminosity. . . . . . 16

2.3 Initial Decay of the $\Upsilon(4 S) \rightarrow B^{+} B^{-}$. . . . . . . . . . . . . 17

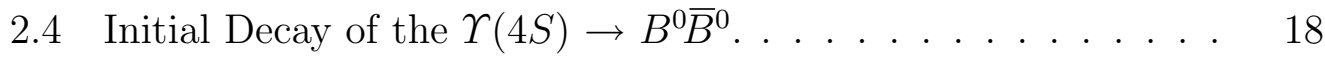

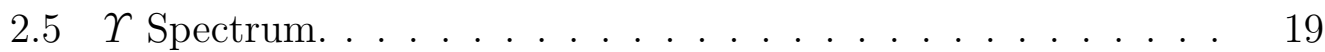

2.6 End view of the SVT. . . . . . . . . . . . . . . . 20

2.7 Side view of the SVT. . . . . . . . . . . . . . . . . 21

2.8 Longitudinal section of the Drift Chamber. . . . . . . . . . . . 21 
2.9 Measurement of $\frac{d E}{d x}$ in the Drift Chamber as a function of track momentum. ..................... 22

2.10 Cerenkov Angle. . . . . . . . . . . . . . . . 23

2.11 Longitudinal section of the EMC. . . . . . . . . . . . . . . 24

2.12 Electromagnetic Calorimeter Interior. . . . . . . . . . . . 25

2.13 Instrumented Flux Return Section. . . . . . . . . . . . . . 26

3.1 Lepton family [55]. . . . . . . . . . . . . . . 30

3.2 Common Mesons [55]. . . . . . . . . . . . . . 31

3.3 Feynman Diagrams of $D_{J}^{0}$ creation. . . . . . . . . . . . 34

4.1 $D^{0} \rightarrow K^{-} \pi^{+}$Sample $\Delta E$ plot showing the Gaussian and linear components. . . . . . . . . . . . . . . 52

4.2 Dalitz weight distribution [63]. . . . . . . . . . . 56

4.3 Kaon momentum spectrum of $B^{-} \rightarrow D^{*+} \pi^{-} \pi^{-}\left(D^{0} \rightarrow K^{-} \pi^{+}\right)$.

4.4 The $\pi^{0}$ momentum spectrum of the data for the decay of $B^{-} \rightarrow$ $D^{*+} \pi^{-} \pi^{-}\left(D^{0} \rightarrow K^{-} \pi^{+} \pi^{0}\right) \ldots \ldots \ldots \ldots$

5.1 The $D^{0}$ mass distribution of $D^{0} \rightarrow K^{-} \pi^{+}$. . . . . . . . . . 73

5.2 The $D^{0}$ mass distribution of $D^{0} \rightarrow K^{-} \pi^{+} \pi^{0}$. . . . . . . . . 74

5.3 The $D^{0}$ mass distribution of $D^{0} \rightarrow K^{-} \pi^{+} \pi^{-} \pi^{+}$. . . . . . . . 75

5.4 The $D^{+}$mass distribution of $D^{+} \rightarrow K^{-} \pi^{+} \pi^{+}$. . . . . . . . 76 
$5.5 \Delta\left(m\left(D^{*+}\right)-m\left(D^{0}\right)\right)$ mass difference with $D^{0} \rightarrow K^{-} \pi^{+}$. . . 76

$5.6 \Delta\left(m\left(D^{*+}\right)-m\left(D^{0}\right)\right)$ mass difference with $D^{0} \rightarrow K^{-} \pi^{+} \pi^{0} . . \quad 77$

$5.7 \Delta\left(m\left(D^{*+}\right)-m\left(D^{0}\right)\right)$ mass difference with $D^{0} \rightarrow K^{-} \pi^{+} \pi^{-} \pi^{+} . \quad 77$

5.8 The $m_{\mathrm{ES}}$ distribution using $D^{0} \rightarrow K^{-} \pi^{+} \ldots \ldots$. . . . . . . . 79

5.9 The $m_{\mathrm{ES}}$ distribution using $D^{0} \rightarrow K^{-} \pi^{+} \pi^{0}$. . . . . . . . . . 79

5.10 The $m_{\mathrm{ES}}$ distribution using $D^{0} \rightarrow K^{-} \pi^{+} \pi^{-} \pi^{+} \ldots$. . . . . . . 80

5.11 The $m_{\mathrm{ES}}$ distribution using $D^{+} \rightarrow K^{-} \pi^{+} \pi^{+}$. . . . . . . . . . 80

5.12 The $\Delta E$ distribution using $D^{0} \rightarrow K^{-} \pi^{+}$. . . . . . . . . . . . 81

5.13 The $\Delta E$ distribution using $D^{0} \rightarrow K^{-} \pi^{+} \pi^{0} \ldots$. . . . . . . . . 81

5.14 The $\Delta E$ distribution using $D^{0} \rightarrow K^{-} \pi^{+} \pi^{-} \pi^{+}$. . . . . . . . . 82

5.15 The $\Delta E$ distribution using $D^{+} \rightarrow K^{-} \pi^{+} \pi^{+}$. . . . . . . . 82

5.16 The $\Delta E$ distribution in the signal subtracted $B \bar{B} \mathrm{MC}$ using the decays of $B^{-} \rightarrow D^{*+} \pi^{-} \pi^{-}\left(D^{0} \rightarrow K^{-} \pi^{+}, D^{0} \rightarrow K^{-} \pi^{+} \pi^{0}\right.$ and $\left.D^{0} \rightarrow K^{-} \pi^{+} \pi^{-} \pi^{+}\right)$. The yield for the peaking background in the MC sample is the area between the red and the dashed blue curve. ........................ 86 
5.17 The $\Delta E$ distribution in the signal subtracted $q \bar{q} \mathrm{MC}$ using the decays of $B^{-} \rightarrow D^{*+} \pi^{-} \pi^{-}\left(D^{0} \rightarrow K^{-} \pi^{+}, D^{0} \rightarrow K^{-} \pi^{+} \pi^{0}\right.$ and $D^{0} \rightarrow K^{-} \pi^{+} \pi^{-} \pi^{+}$). The yield for the peaking background in the MC sample is the area between the red and the dashed blue

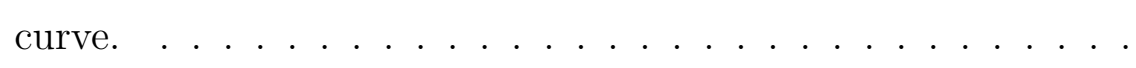

5.18 The $\Delta E$ distribution in the signal subtracted combined MC ( $q \bar{q}$ and $B \bar{B})$ using the decays of $B^{-} \rightarrow D^{*+} \pi^{-} \pi^{-}\left(D^{0} \rightarrow K^{-} \pi^{+}\right.$, $D^{0} \rightarrow K^{-} \pi^{+} \pi^{0}$ and $D^{0} \rightarrow K^{-} \pi^{+} \pi^{-} \pi^{+}$). The yield for the peaking background in the MC sample is the area between the red and the dashed blue curve. . . . . . . . . . . .

5.19 The $\Delta E$ distribution in the signal subtracted $B \bar{B}$ MC using the decays of $B^{-} \rightarrow D^{+} \pi^{-} \pi^{-}\left(D^{+} \rightarrow K^{-} \pi^{+} \pi^{+}\right)$. The yield for the peaking background in the MC sample is the area between the red and the dashed blue curve. . . . . . . . . . . .

5.20 The $\Delta E$ distribution in the signal subtracted $q \bar{q}$ MC using the decays of $B^{-} \rightarrow D^{+} \pi^{-} \pi^{-}\left(D^{+} \rightarrow K^{-} \pi^{+} \pi^{+}\right)$. The yield for the peaking background in the MC sample is the area between the red and the dashed blue curve. . . . . . . . . . . 
5.21 The $\Delta E$ distribution in the signal subtracted combined MC $(q \bar{q}$ and $B \bar{B})$ using the decays of $B^{-} \rightarrow D^{+} \pi^{-} \pi^{-}\left(D^{+} \rightarrow K^{-} \pi^{+} \pi^{+}\right)$. The yield for the peaking background in the MC sample is the area between the red and the dashed blue curve. . . . . . . 91

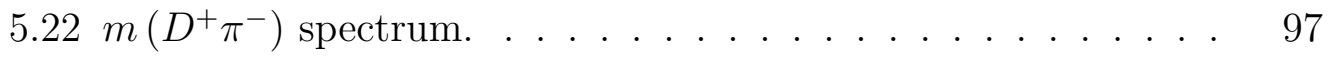

$5.23 m\left(D^{+} \pi^{-}\right)$spectrum, $\Delta E$ Sideband. . . . . . . . . . 98

$5.24 m\left(D^{*+} \pi^{-}\right)$spectrum, $\Delta E$ Sideband. . . . . . . . . . 99

$5.25 m\left(D^{*+} \pi^{-}\right)$spectrum. . . . . . . . . . . . . . 100 


\section{Chapter 1}

\section{Introduction}

The BABAR experiment provides an unprecedented opportunity to study the production and decay of the $D_{J}^{0}$ charmed mesons. The subject of this thesis is the B-meson decay $B^{-} \rightarrow D^{+(*)} \pi^{-} \pi^{-}$for which the dominant decay contribution comes through the intermediate $D_{J}^{0}$ resonances.

\subsection{The $D_{J}^{0}$ Spectroscopy}

There are six known quarks (q) [1,2] all of which are believed to be fundamental particles. They are: up, down, charm, strange, top, and bottom [3]. Table 1.1 lists the properties of the six quarks. Hadrons are particles that consist of two or three quarks. Mesons contain a quark and an anti-quark while baryons contain three quarks. 
$D_{J}^{0}$ refers to a group of four orbitally excited $D^{0}$ mesons $[4,5,6,7$, $8,9,10,11,12,13,14,18,19]$. The other commonly used name for $D_{J}^{0}$ is $D^{* * 0}$. They have an orbital angular momentum of $L=1$. The measurement of the properties such as decay width and branching fraction of these particles are important validations of Heavy Quark Effective Theory (HQET). HQET is an effective theory which assumes Heavy Quark Symmetry (HQS). This assumption is that the charm quark is much heavier than the other quark in the meson $\left(m_{c} \gg \Lambda_{Q C D}\right)[15,16]$.

\begin{tabular}{|c|c|c|c|c|}
\hline & Name & Symbol & Charge (e) & Mass $\left(\mathrm{MeV} / \mathrm{c}^{2}\right)$ \\
\hline Generation I & $\mathrm{Up}$ & $\mathrm{u}$ & $\frac{2}{3}$ & 1.5 to 4 \\
& Down & $\mathrm{d}$ & $-\frac{1}{3}$ & 4 to 8 \\
\hline \multirow{2}{*}{ Generation II } & Charm & $\mathrm{c}$ & $\frac{2}{3}$ & 1150 to 1350 \\
& Strange & $\mathrm{s}$ & $-\frac{1}{3}$ & 80 to 130 \\
\hline \multirow{2}{*}{ Generation III } & Top & $\mathrm{t}$ & $\frac{2}{3}$ & 174000 to 178000 \\
& Bottom & $\mathrm{b}$ & $-\frac{1}{3}$ & 4100 to 4900 \\
\hline
\end{tabular}

Table 1.1: Properties of the Six Known Quarks.

With this assumption, the $D_{J}^{0}$ mesons resemble the hydrogen atom with regard to their angular momentum coupling. The charm quark's spin $S_{c}$ separates from L, the orbital angular momentum as shown in Figure 1.1. The sum $j=L+S_{q}$, total angular momentum of the light quark degrees of freedom 


\section{$L=1 D$-mesons}
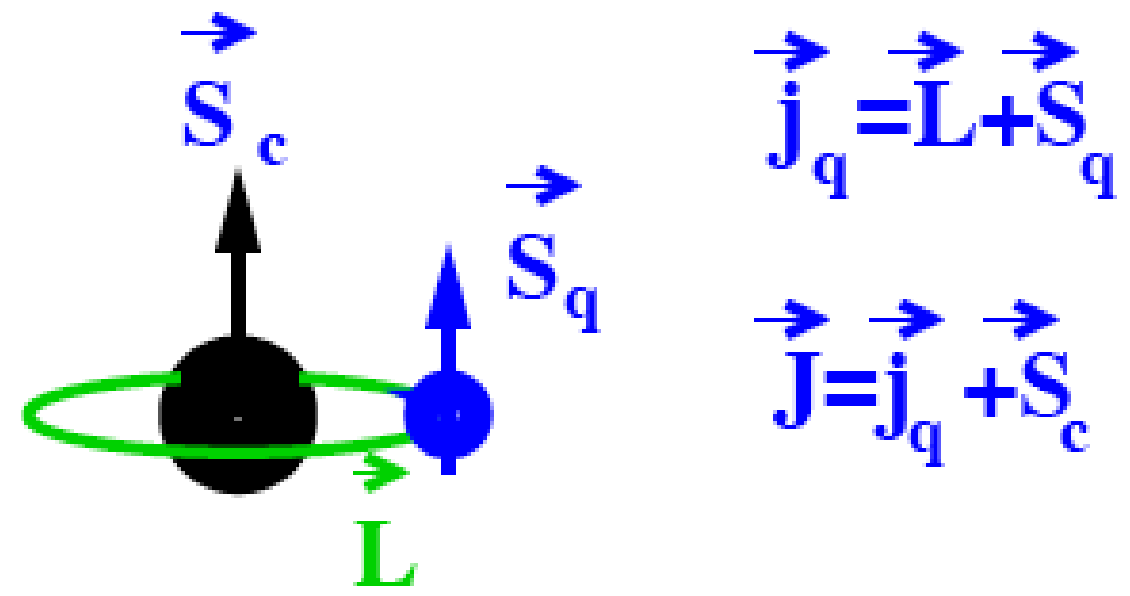

Figure 1.1: Heavy Quark Effective Theory (HQET).

$\left(j_{q}\right.$ in Figure 1.1), is then conserved as a good quantum number. As a result, there are two sets of doublets. One with $j=\frac{3}{2}$ and a second one with $j=\frac{1}{2}$.

On the other hand, if in place of HQET one assumes that the charm mass is small and comparable mass to that of the other quark, $D_{J}^{0}$ is similar to positronium. This allows for a singlet with $J=0$ and $S=0$ and a triplet with $J=0,1,2$ and $S=1$. Therefore, a different spectrum of particles is expected. When $J=0, j=\frac{1}{2}$. When $J=2, j=\frac{3}{2}$. And when $J=1, \mathrm{j}$ is a mixture of 


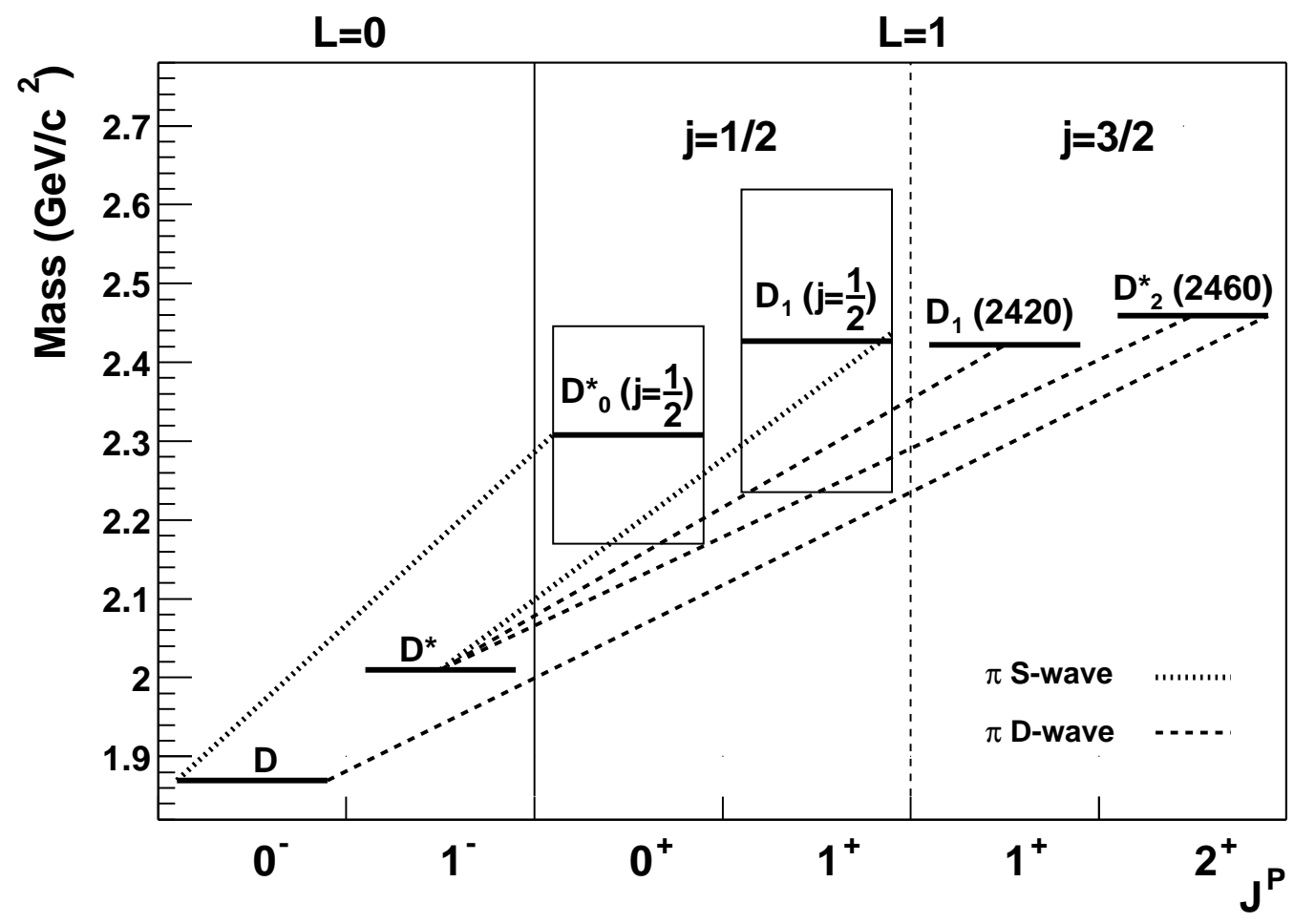

Figure 1.2: Spectroscopy of $D_{J}^{0}$. This figure shows the allowed $\pi$ transitions of $D_{J}^{0}$.

$j=\frac{1}{2}$ and $j=\frac{3}{2}$. Different decay channels would be expected.

From the measurement of the actual masses and widths of the $D_{J}^{0}$, one may determine how applicable the HQET theory predictions are for $D_{J}^{0}$ spectroscopy. The four neutral states are $D_{2}^{*}(2460)^{0}, D_{1}(2420)^{0}, D_{1}\left(j=\frac{1}{2}\right)^{0}$, and $D_{0}^{*}\left(j=\frac{1}{2}\right)^{0}$. The $D_{J}^{0}$ properties are listed in Table 1.2. In addition to the neutral $D_{J}^{0}$, there exists the charged $D_{J}$ spectroscopy. This dissertation limits itself to only $D_{J}^{0}$ particles.

The allowed transitions are restricted because of the conservation of 
parity and angular momentum. This is why two of the resonances are wide and two are narrow. The $D_{2}^{*}(2460)^{0}$ must decay via a D wave and the $D_{0}^{*}\left(j=\frac{1}{2}\right)^{0}$ must decay via the $\mathrm{S}$ wave. If HQS is applicable, $D_{1}(2420)^{0}$ must decay via the $\mathrm{S}$ wave and $D_{1}\left(j=\frac{1}{2}\right)^{0}$ must decay via the D wave. Figure 1.2 and Table 1.2 show the spectroscopy of $D_{J}^{0}$. The two $D_{J}^{0}$ mesons with $j=\frac{1}{2}$ states have broad (a few hundred $\mathrm{MeV}$ ) resonances while the two $j=\frac{3}{2}$ states have narrow resonances (20-30 MeV). Further discussion of the S and D wave decays is presented in Chapter 3 .

\begin{tabular}{|ll|lccccc|}
\hline $\begin{array}{l}\text { Common Convention } \\
\text { Type 1 }\end{array}$ & Type 2 & $J^{P}$ & $\begin{array}{c}\text { Mass } \\
\left(\mathrm{MeV} / c^{2}\right)\end{array}$ & $\begin{array}{c}\text { Width } \\
\left(\mathrm{MeV} / c^{2}\right)\end{array}$ & $\begin{array}{c}\text { Decay } \\
\text { Mode }\end{array}$ & $\begin{array}{c}\text { Partial } \\
\text { wave }\end{array}$ & $\begin{array}{c}\text { HQS } \\
\text { allowed }\end{array}$ \\
\hline$D_{0}^{*}(j=1 / 2)^{0}$ & $D_{J}^{0}\left(0_{1 / 2}^{+}\right)$ & $0^{+}$ & - & - & $D \pi$ & $\mathrm{s}$ & $\mathrm{s}$ \\
$D_{1}(2420)^{0}$ & $D_{J}^{0}\left(1_{3 / 2}^{+}\right)$ & $1^{+}$ & $2422.2 \pm 1.8$ & $18.9_{-3.5}^{+4.6}$ & $D^{*} \pi$ & $\mathrm{s}, \mathrm{d}$ & $\mathrm{d}$ \\
$D_{1}(j=1 / 2)^{0}$ & $D_{J}^{0}\left(1_{1 / 2}^{+}\right)$ & $1^{+}$ & - & - & $D^{*} \pi$ & $\mathrm{s}, \mathrm{d}$ & $\mathrm{s}$ \\
$D_{2}^{*}(2460)^{0}$ & $D_{J}^{0}\left(2_{3 / 2}^{+}\right)$ & $2^{+}$ & $2458.9 \pm 2.0$ & $23 \pm 5$ & $D^{*} \pi, D \pi$ & $\mathrm{d}$ & $\mathrm{d}$ \\
\hline
\end{tabular}

Table 1.2: Properties of $D_{J}^{0}$ spectroscopy including their mass, width and allowed transitions.

The CLEO (Ithaca, New York) [6, 7, 9] and BELLE (Japan) [12] experiments have studied $D_{J}^{0}$. One common parameter both collaborations have measured is the ratio of the two branching fractions of the two narrow $D_{J}^{0}$ states defined as:

$$
R \equiv \frac{\mathcal{B}\left(B^{-} \rightarrow D_{2}^{*}(2460)^{0} \pi^{-}\right)}{\mathcal{B}\left(B^{-} \rightarrow D_{1}(2420)^{0} \pi^{-}\right)}
$$




\begin{tabular}{|c|c|c|c|}
\hline $\mathrm{BF}\left(10^{-3}\right)$ & CLEO $[6,7,9]$ & BELLE [12] & $\mathrm{PDG}[80]$ \\
\hline $\begin{array}{l}B^{-} \rightarrow D^{*+} \pi^{-} \pi^{-} \\
B^{-} \rightarrow D^{+} \pi^{-} \pi^{-}\end{array}$ & $\begin{array}{c}1.9 \pm 0.7 \pm 0.3 \\
<1.4(90 \% \text { C.L. })\end{array}$ & $\begin{array}{l}1.24 \pm 0.07 \pm 0.22 \\
1.07 \pm 0.05 \pm 0.16\end{array}$ & $\begin{array}{c}2.1 \pm 0.6 \\
<1.4(90 \% \text { C.L. })\end{array}$ \\
\hline $\begin{array}{l}\left(B^{-} \rightarrow D_{1}(2420)^{0} \pi^{-}\right) \\
\quad \times\left(D_{1}(2420)^{0} \rightarrow D^{*+} \pi^{-}\right) \\
\left(B^{-} \rightarrow D_{2}^{*}(2460)^{0} \pi^{-}\right) \\
\quad \times\left(D_{2}^{*}(2460)^{0} \rightarrow D^{*+} \pi^{-}\right) \\
\left(B^{-} \rightarrow D_{1}(j=1 / 2)^{0} \pi^{-}\right) \\
\quad \times\left(D_{1}(j=1 / 2)^{0} \rightarrow D^{*+} \pi^{-}\right)\end{array}$ & $\begin{array}{c}0.69_{-0.14}^{+0.18} \pm 0.12 \\
0.31 \pm 0.08 \pm 0.05 \\
1.06 \pm 0.19 \pm 0.29\end{array}$ & $\begin{array}{l}0.68 \pm 0.07 \pm 0.13 \pm 0.03 \\
0.18 \pm 0.03 \pm 0.03 \pm 0.02 \\
0.50 \pm 0.04 \pm 0.10 \pm 0.04\end{array}$ & - \\
\hline $\begin{array}{l}\left(B^{-} \rightarrow D_{2}^{*}(2460)^{0} \pi^{-}\right) \\
\quad \times\left(D_{2}^{*}(2460)^{0} \rightarrow D^{+} \pi^{-}\right) \\
\left(B^{-} \rightarrow D_{0}^{* 0} \pi^{-}\right) \\
\quad \times\left(D_{0}^{* 0} \rightarrow D^{+} \pi^{-}\right)\end{array}$ & - & $\begin{array}{l}0.34 \pm 0.03 \pm 0.06 \pm 0.04 \\
0.61 \pm 0.06 \pm 0.09 \pm 0.16\end{array}$ & - \\
\hline \multicolumn{4}{|c|}{$D_{J}$ Mass and Width $(\mathrm{MeV})$} \\
\hline $\begin{array}{l}m\left(D_{0}^{*}(j=1 / 2)^{0}\right) \\
\Gamma\left(D_{0}^{*}(j=1 / 2)^{0}\right)\end{array}$ & - & $\begin{array}{c}2308 \pm 17 \pm 15 \pm 20 \\
276 \pm 21 \pm 18 \pm 60\end{array}$ & - \\
\hline $\begin{array}{l}m\left(D_{1}(2420)\right) \\
\Gamma\left(D_{1}(2420)\right)\end{array}$ & $\begin{array}{c}2421_{-1}^{+1} \pm 2 \\
20_{-5}^{+6} \pm 3 \\
\end{array}$ & $\begin{array}{c}2421.4 \pm 2.0 \pm 0.4 \pm 0.8 \\
23.7 \pm 2.7 \pm 0.2 \pm 4.0\end{array}$ & $\begin{array}{c}2422.2 \pm 1.8 \\
18.9_{-3.5}^{+4.6} \\
\end{array}$ \\
\hline $\begin{array}{l}m\left(D_{1}(j=1 / 2)^{0}\right) \\
\Gamma\left(D_{1}(j=1 / 2)^{0}\right)\end{array}$ & $\begin{array}{l}2461_{-34}^{+41} \pm 34 \\
290_{-79}^{+101} \pm 44\end{array}$ & $\begin{array}{c}2400 \pm 30 \pm 20 \\
380 \pm 100 \pm 100\end{array}$ & - \\
\hline $\begin{array}{l}m\left(D_{2}^{*}(2460)^{0}\right) \\
\Gamma\left(D_{2}^{*}(2460)^{0}\right)\end{array}$ & $\begin{array}{c}2465 \pm 3 \pm 3 \\
28_{-7}^{+8} \pm 6\end{array}$ & $\begin{array}{c}2461.6 \pm 2.1 \pm 0.5 \pm 3.3 \\
45.6 \pm 4.4 \pm 6.5 \pm 1.6\end{array}$ & $\begin{array}{l}2458.9 \pm 2.0 \\
23 \pm 5\end{array}$ \\
\hline
\end{tabular}

Table 1.3: Previous measurements of $\mathcal{B}\left(B^{-} \rightarrow D^{+} \pi^{-} \pi^{-}\right)$and $\mathcal{B}\left(B^{-} \rightarrow D^{*+} \pi^{-} \pi^{-}\right)$.

\begin{tabular}{|c|c|c|}
\hline Neubert & CLEO 98 & Belle 2003 \\
\hline 0.35 & $1.8 \pm 0.8$ & $0.77 \pm 0.15$ \\
\hline
\end{tabular}

Table 1.4: Measurements and Calculation for $\mathrm{R}$.

The HQET based theory prediction by Neubert found $\mathrm{R}=0.35$ [77].

BELLE's and CLEO's results are shown in Table 1.4. Previously published $D_{J}^{0}$ measurements are listed in Table 1.3. 


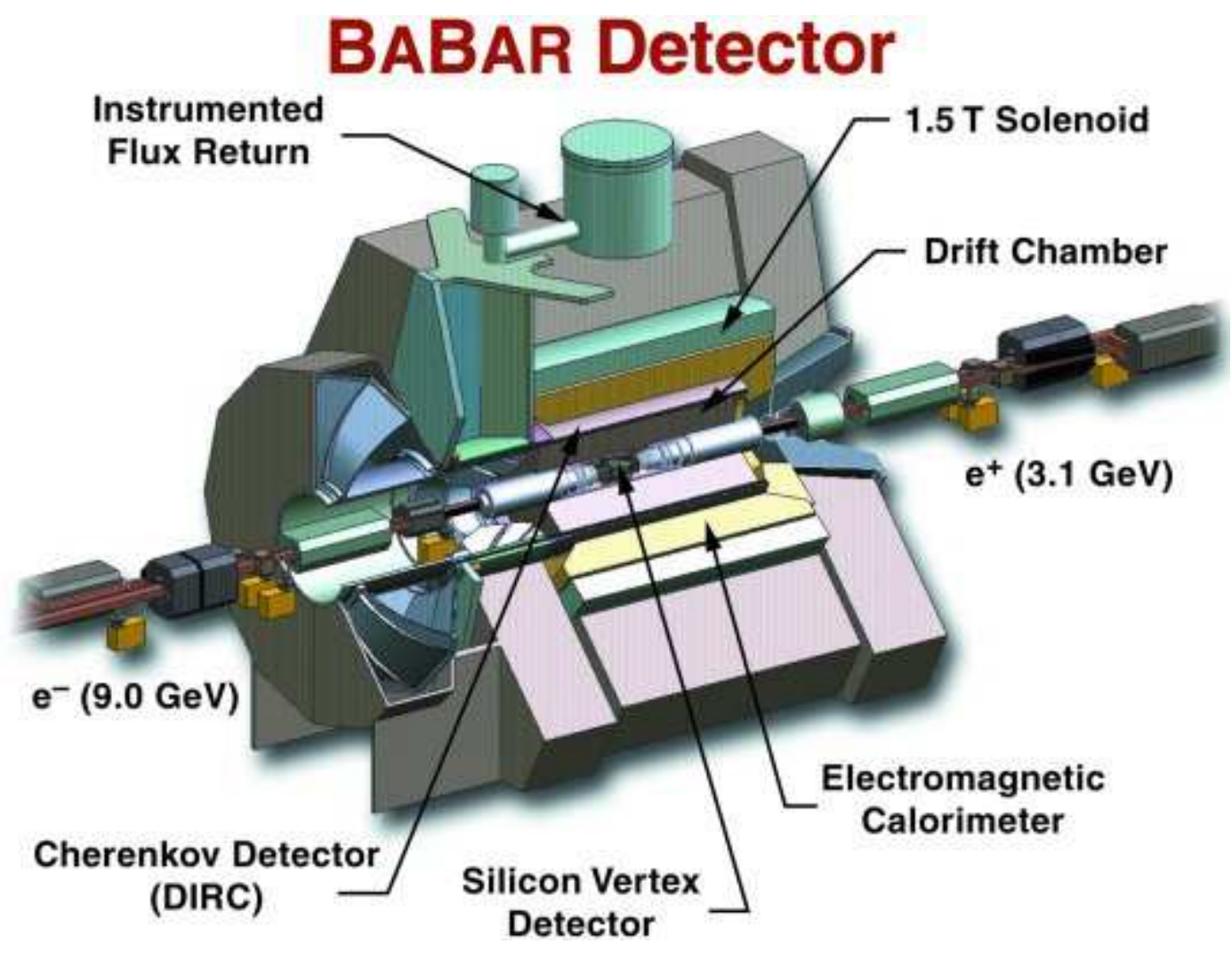

Figure 1.3: The BABAR detector.

\section{$1.2 \quad$ PEP-II and BABAR}

Located at the Stanford Linear Accelerator Center (SLAC) (see Figure

1.5), the BABAR detector (Figure 1.3) has been recording data since 1999, observing the collisions provided by the PEP-II asymmetric $B$ Factory (Figure 1.4). BABAR is named after an elephant from literature [17]. PEP-II runs at the center of mass energy equal to the rest mass of the $\Upsilon(4 S)$ [68] because the $\Upsilon(4 S)$ center of mass is just $22 \mathrm{MeV} / c^{2}$ above the $B^{0} \bar{B}^{0}$ mass threshold. 


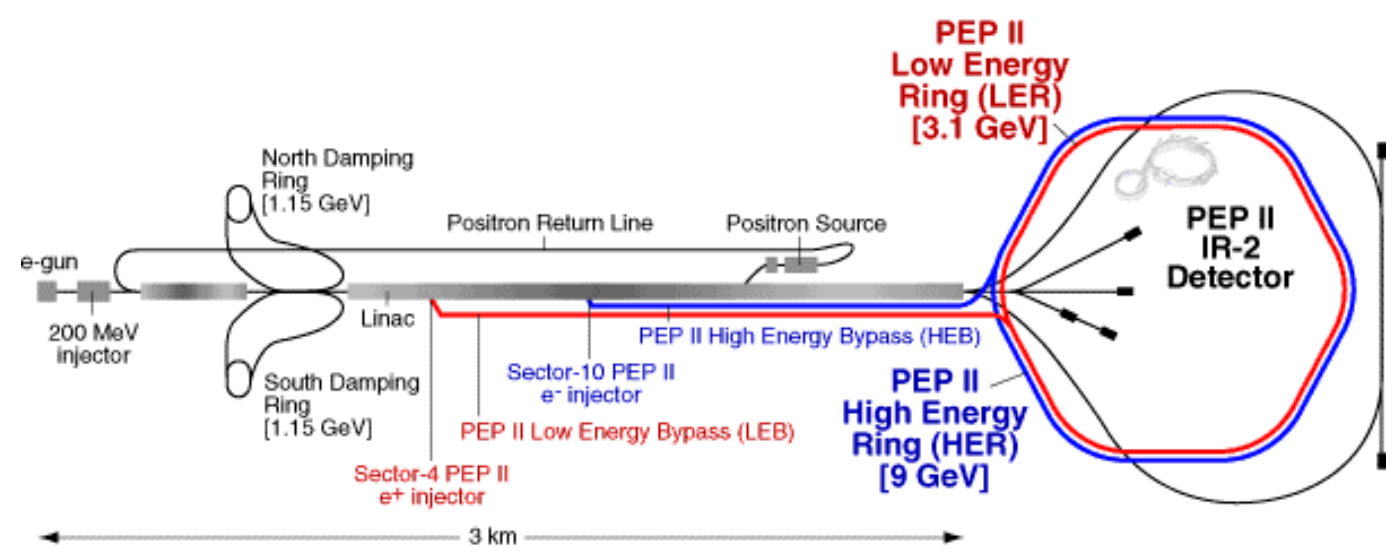

Figure 1.4: SLAC / PEP2.

The $\Upsilon(4 S)$ provides a clean environment for the observation of the B-mesons. $\Upsilon(4 S)$ primarily decays to $B^{+} B^{-}(50 \%)$ and $B^{0} \bar{B}^{0}(50 \%)$ pairs, particles that are useful in the study of CP violation $[74,75,72,67]$.

The BABAR detector [24] consists of several sub-detectors. The silicon vertex detector (SVT) and drift chamber (DCH), used to measure charged particle trajectories lie within a $1.5 \mathrm{~T}$ solenoidal magnetic field [35]. The charged particle identification is done by using the $\frac{d E}{d x}$ information in the DCH and the SVT (Sections 2.3 and 2.4) in conjunction with the opening angle information gathered from the ring-imaging Cerenkov detector (Section 2.5). Surrounding these is the Electromagnetic Calorimeter (EMC). The EMC is used in the identification of the neutral particles such as photons, $\pi^{0}$ 's and $\eta$ 's. The identification of muons and neutral hadrons is done by the Instrumented Flux Return (IFR). Further documentation about the detector is supplied in Chap- 


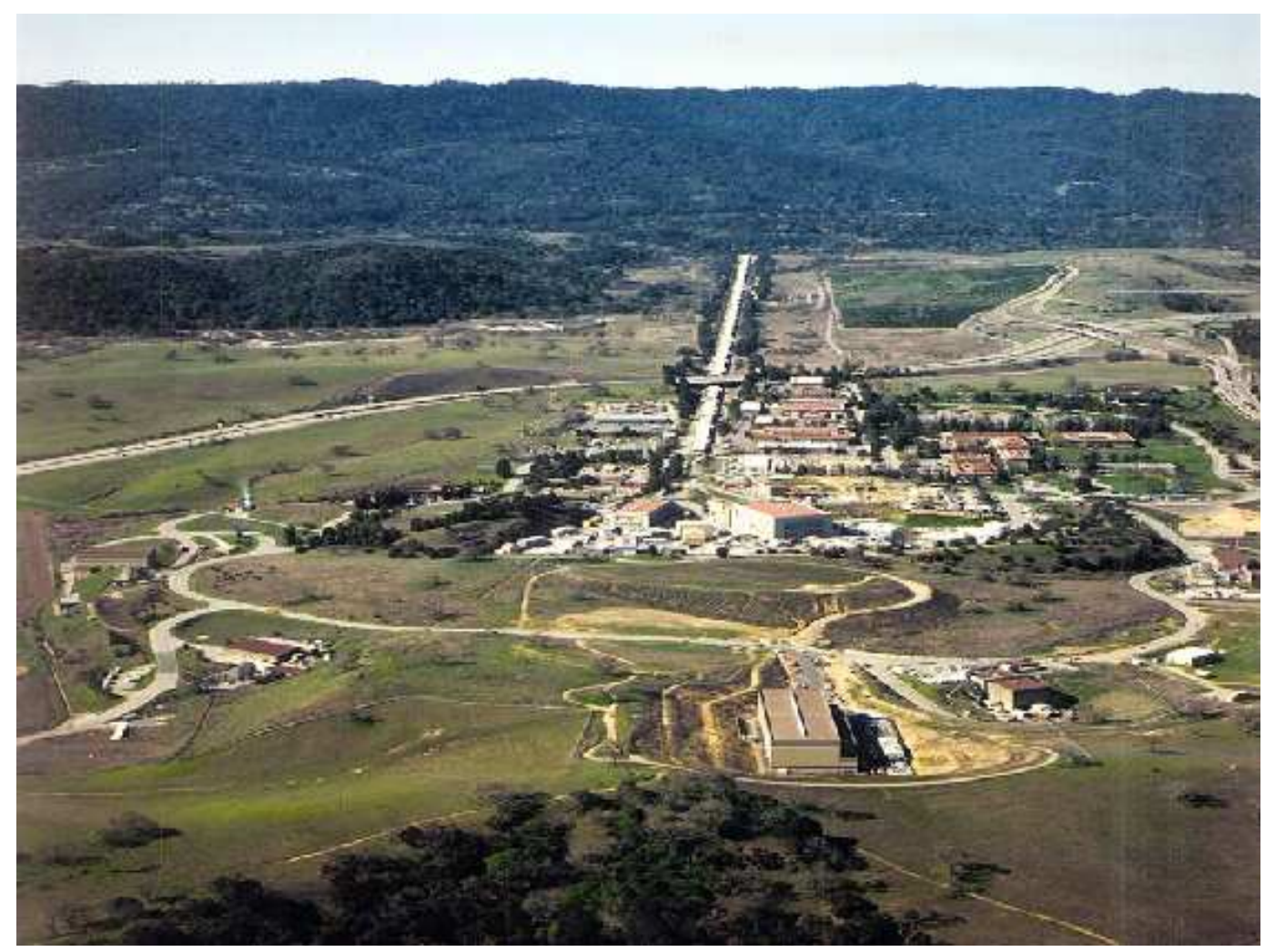

Figure 1.5: Stanford Linear Accelerator Center (SLAC).

ter 2 .

\subsection{Procedure}

The quantities that are the subject of this investigation will be measured through the reconstruction of the $B^{-}$meson via the final states $B^{-} \rightarrow$ $D^{*+} \pi^{-} \pi^{-}$and $B^{-} \rightarrow D^{+} \pi^{-} \pi^{-}$(Table 1.5 ). The $D_{J}^{0}$ sample is contained within these data sets. $D^{*+}$ candidates will be reconstructed with a $D^{0}$ and a $\pi^{+}$. $D^{0}$ candidates will be reconstructed using the following modes: $D^{0} \rightarrow K^{-} \pi^{+}$, 
$D^{0} \rightarrow K^{-} \pi^{+} \pi^{0}, D^{0} \rightarrow K^{-} \pi^{+} \pi^{-} \pi^{+} . D^{+}$candidates will be reconstructed using the $D^{+} \rightarrow K^{-} \pi^{+} \pi^{+}$decays (Table 1.5 ).

In addition to real data, this analysis uses Monte Carlo (MC) data. Without reconstruction of MC data, efficiencies cannot be calculated and the analysis cannot be completed. It is also used for the optimization of selection criteria and to assist in the estimation of the backgrounds by checking which other modes can provide false positive events.

\begin{tabular}{|c|l|}
\hline$B^{-} \rightarrow D^{*+} \pi^{-} \pi^{-}$ & $B^{-} \rightarrow D^{+} \pi^{-} \pi^{-}$ \\
\hline$D^{0} \rightarrow K^{-} \pi^{+}$ & $D^{+} \rightarrow K^{-} \pi^{+} \pi^{+}$ \\
$D^{0} \rightarrow K^{-} \pi^{+} \pi^{0}$ & \\
$D^{0} \rightarrow K^{-} \pi^{+} \pi^{-} \pi^{+}$ & \\
\hline
\end{tabular}

Table 1.5: Reconstructed Mode Chains for $B^{-} \rightarrow D^{+} \pi^{-} \pi^{-}$and $B^{-} \rightarrow D^{*+} \pi^{-} \pi^{-}$.

The measurements this analysis present are:

1. $\mathcal{B}\left(B^{-} \rightarrow D^{+} \pi^{-} \pi^{-}\right)$- Branching fraction for the inclusive mode $B^{-} \rightarrow$ $D^{+} \pi^{-} \pi^{-}$. This includes all relevant $D_{J}^{0}$ particles as well the as nonresonant mode.

2. $\mathcal{B}\left(B^{-} \rightarrow D^{*+} \pi^{-} \pi^{-}\right)$- Branching fraction for the other inclusive mode, $B^{-} \rightarrow D^{*+} \pi^{-} \pi^{-}$ 


\section{Chapter 2}

\section{BABAR Detector and PEP-II}

\section{$2.1 \quad$ Introduction}

The BABAR detector (Figure 2.1) [24] provides the full reconstruction of $B \bar{B}$ events in the PEP-II collider. This enables BABAR's study of CP violation and heavy flavor physics. An electron and a positron collide at the Interaction Point (IP) inside the BABAR detector to form the $\Upsilon(4 S)$ resonance state [68], which decays $100 \%$ of the time to $B \bar{B}$ pairs. The mass of $\Upsilon(4 S)$ is about $22 \mathrm{MeV}$ above the mass threshold for $B \bar{B}$ pairs, either $B^{+} B^{-}(50 \%)$ or $B^{0} \bar{B}^{0}(50 \%)$, with no possibility of additional pions or any type of $\mathrm{b}$ hadrons. $B A B A R$ has measured the branching fraction of $\Upsilon(4 S) \rightarrow B^{0} \bar{B}^{0}$ directly [69]. It is: 


$$
f_{00} \equiv \mathcal{B}\left(\Upsilon(4 S) \rightarrow B^{0} \bar{B}^{0}\right)=0.487 \pm 0.010(\text { stat }) \pm 0.008(\text { sys })[70] .
$$

There is no direct measurement of the branching fraction $f_{+-} \equiv \mathcal{B}(\Upsilon(4 S)$ $\rightarrow B^{+} B^{-}$). However, using the world average of $\frac{f_{+-}}{f_{00}}=1.020 \pm 0.034[71]$ and assuming:

$$
f_{+-}+f_{00}=1
$$

it is calculated that:

$$
f_{00}=0.493 \pm 0.007
$$

and:

$$
f_{+-}=0.507 \pm 0.007 .
$$

By adding $f_{00}$ as listed in [70], the current world average is now:

$$
\frac{f_{+-}}{f_{00}}=1.030 \pm 0.029
$$

The subsequent decay chains are observed and recorded by BABAR's sub- 


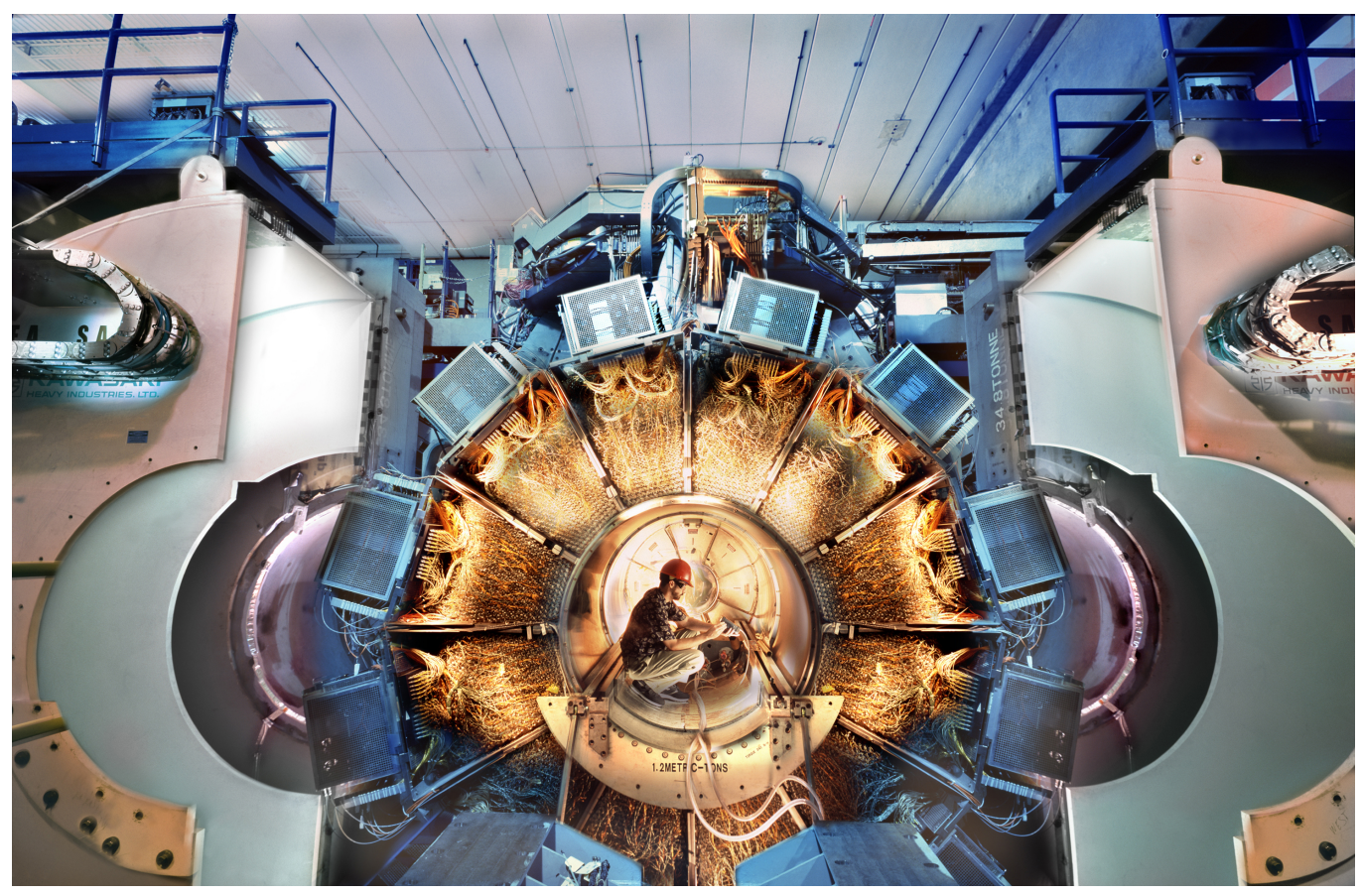

Figure 2.1: Opened view of $B A B A R$.

detectors:

1. Silicon Vertex Detector (SVT)

2. Drift Chamber (DCH)

3. Detector of Internally Reflected Cerenkov Light (DIRC)

4. Electromagnetic Calorimeter (EMC)

5. Instrumented Flux Return (IFR).

The collision event is digitized and then a subsystem called the trigger makes a decision about the quality of the event. If the trigger accepts it, the event is 
passed along to reconstruction and then storage. This chapter describes the process of the observation of the event.

\subsection{The Asymmetry B-Factory, PEP-II}

PEP-II is an $e^{+} e^{-}$storage ring $[22,21,20,23]$ at the Stanford Linear Accelerator Center (SLAC) (See Figure 1.4). PEP-II operates at an energy of $\sqrt{s}=10.580 \mathrm{GeV}$, the mass of the $\Upsilon(4 S)$ resonance. The beam has an energy RMS spread of 4.6 MeV. The electrons are stored in the High Energy Ring (HER) at an energy of $9.0 \mathrm{GeV}$. The positrons are stored in the Low Energy Ring (LER) at an energy of $3.1 \mathrm{GeV}$. Approximately $12 \%$ of the time, data is taken at $40 \mathrm{MeV}$ below the resonance to allow studies of the non-resonant background (light quark continuum) in the data. This consists of $q \bar{q}$ events where $q$ is either a $\mathrm{u}, \mathrm{d}, \mathrm{c}$, or s quark.

When the particles collide at the Interaction Point (IP), an $\Upsilon(4 S)$, a $b \bar{b}$ resonance, is formed. It is part of the $\Upsilon$ particle family whose mass spectrum is shown in Figure 2.5. The mass of the $\Upsilon(4 S)$ is just above the rest mass for a $B \bar{B}$ pair as shown in Figures 2.3 and 2.4. The decay of the $\Upsilon(4 S)$ produces very little kinetic energy. Therefore, the mesons are produced virtually at rest in the rest frame of the $\Upsilon(4 S)$. Because of the asymmetry of the energies of the beams, there exists a Lorentz boost of $\beta \gamma=0.56$ with respect to the 
laboratory frame.

This boost allows one to measure the difference of the decay times of the two B mesons produced at $\Upsilon(4 S)$. This information is needed for measuring time-dependent CP studies. With this boost the average difference between decay times for two $\mathrm{B}$ mesons translates to an average separation of the $\mathrm{B}$ decay vertices of $|\Delta z|=250 \mu \mathrm{m}$. The typical cross-section of $b \bar{b}$ at the $\Upsilon(4 S)$ resonance is $1.05 \mathrm{nb}$ with typical average luminosities of $10^{33} \mathrm{~cm}^{-2} \mathrm{~s}^{-1}$. The current PEP-II delivered and recorded luminosity is shown in Figure 2.2.

\subsection{Silicon Vertex Detector (SVT)}

The SVT [25, 26, 27, 28] (Figures 2.6 and 2.7) is designed to measure the trajectories of charged particles in the region closest to the interaction point. Having hit information very close to the IP allows one to locate the B meson decay vertices. It is also helpful for the reconstruction of tracks with a low momentum.

This sub-detector is composed of several layers of silicon strip detectors. The detectors are double-sided silicon strips with a readout at each end. Strips on the opposite sides of each layer are perpendicular. Digitization is performed 


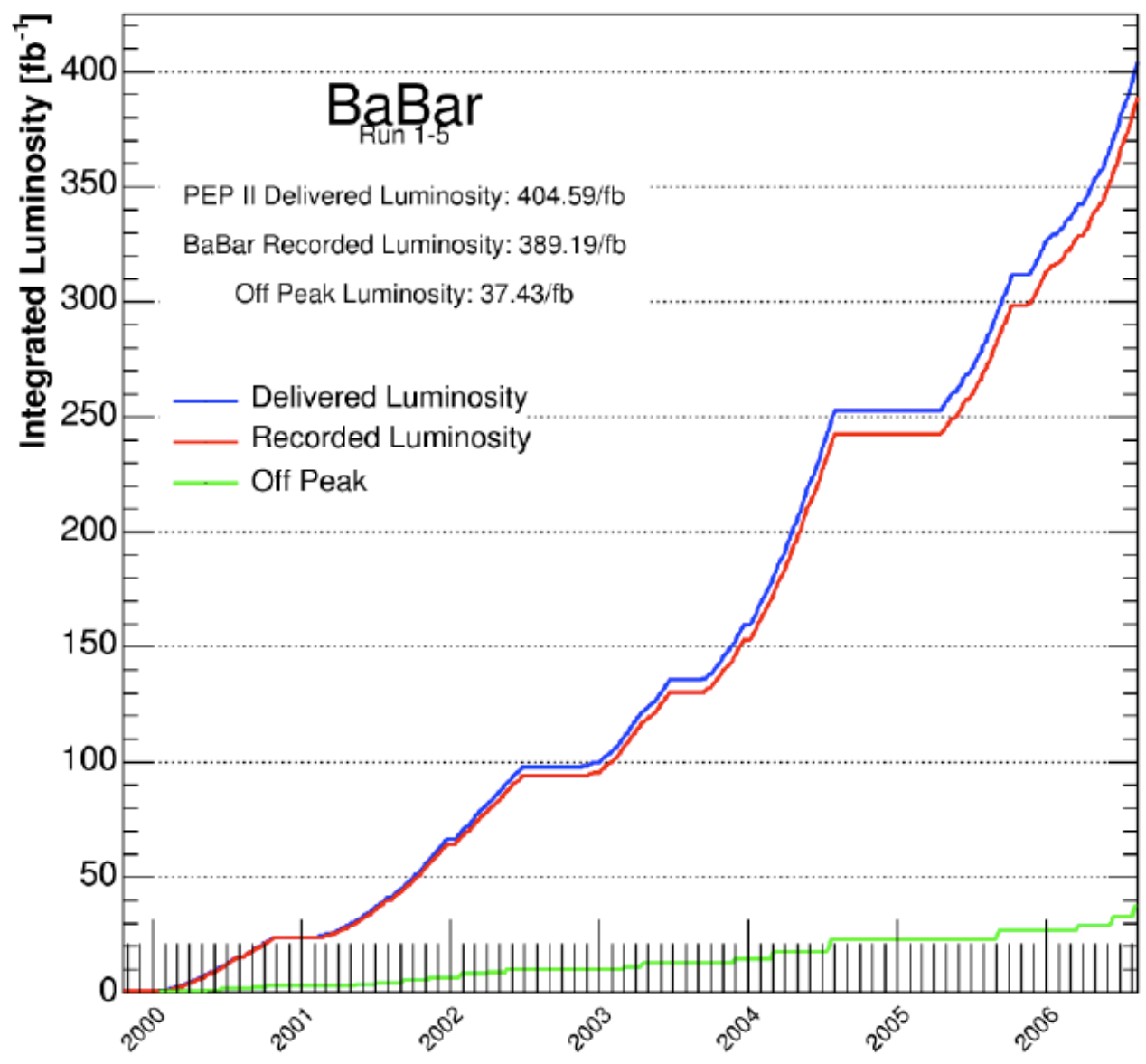

Figure 2.2: PEP-II Delivered and BABAR Integrated Luminosity.

by an ATOM ("A Time-Over-Threshold Machine") chip. One chip is at the end of each set of 128 strips. The ATOM chip amplifies, digitizes, and stores the signal from each channel. 


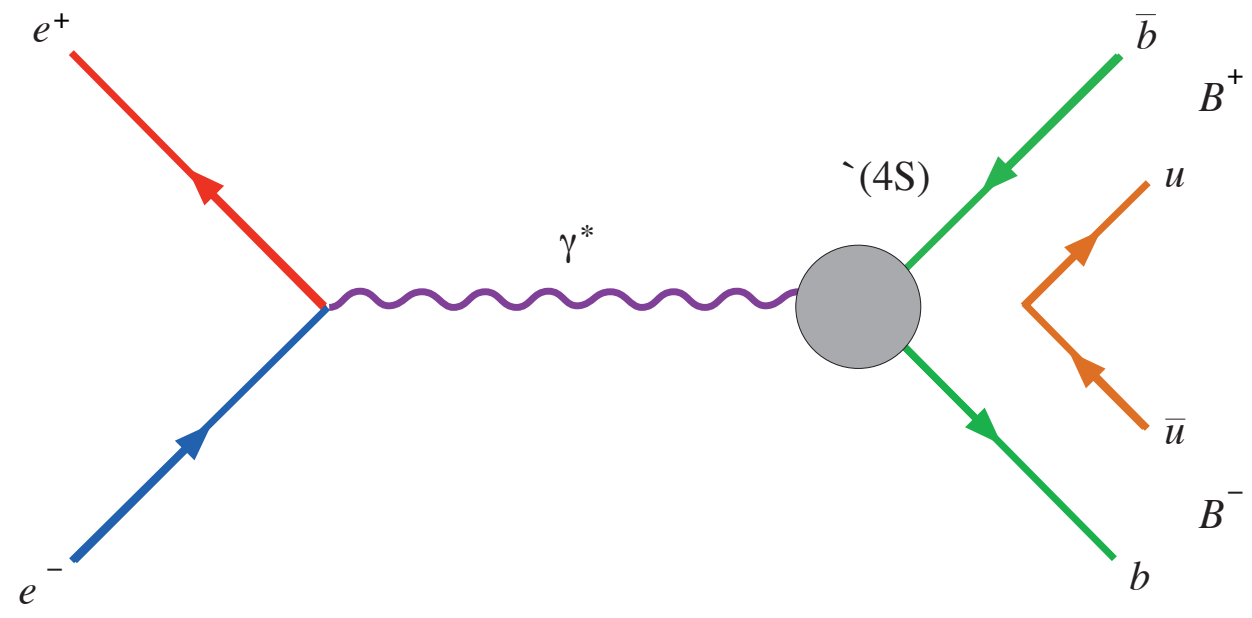

Figure 2.3: Initial Decay of the $\Upsilon(4 S) \rightarrow B^{+} B^{-}$.

\subsection{Drift Chamber (DCH)}

The Drift Chamber (DCH) (Figure 2.8) is another sub-detector that detects charged particle trajectories. As a charged particle passes through the DCH [29], it leaves an ionization trail. The ionization charge drifts to sense wires due to strong local electric fields. The drift time, the time required for the charge to drift to the wire, is a function of the distance of closest approach (DOCA) of the track to the wire. The signals from the wires are digitized. A pattern recognition program determines which hits belong to the same trajectory. A fit to the particle trajectory is performed. It is the ionization charge per unit length that gives information about the particles mass. 


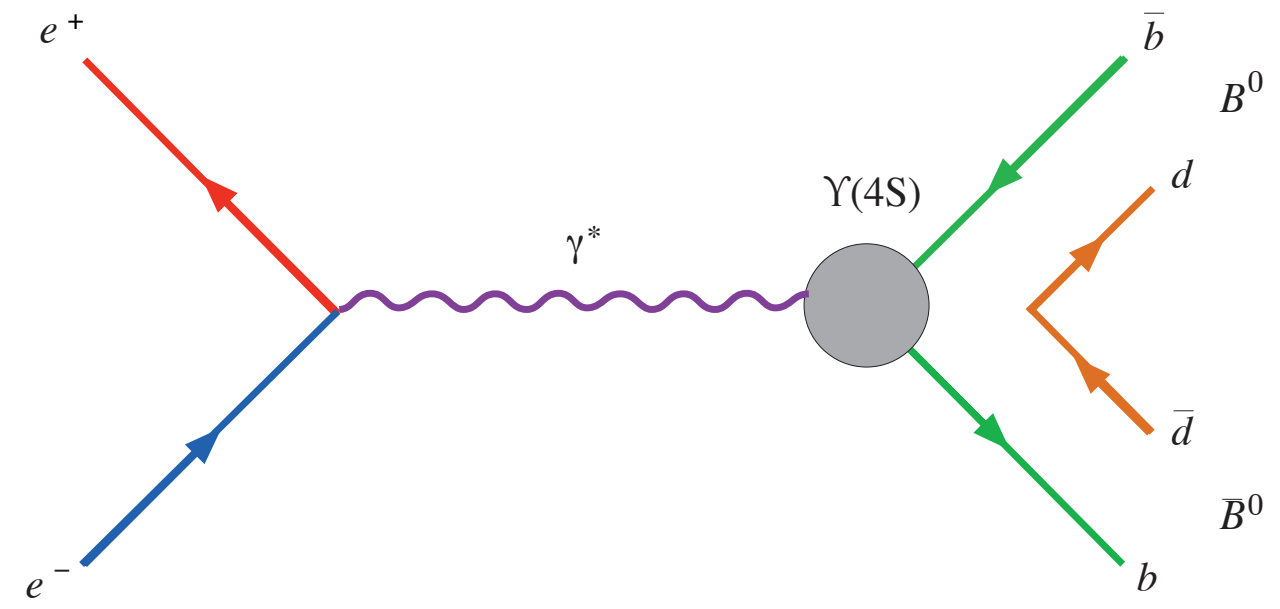

Figure 2.4: Initial Decay of the $\Upsilon(4 S) \rightarrow B^{0} \bar{B}^{0}$.

The DCH contains 40 layers of gold-coated tungsten rhenium sense wires and gold-coated aluminum wires. These wires are contained in a mixture of $80 \%$ helium and $20 \%$ isobutane gas. The helium is used to reduce multiple scattering $[29,30]$. The layers are grouped by fours into 10 sets of layers. The sets are variously oriented; some are parallel to the beam axis. Others are at a slight angle with respect to the beam axis.

The energy loss per unit length of tracks, $\frac{d E}{d x}$, contains particle type information due to the dependence of $\frac{d E}{d x}$ on particle velocity according to the Bethe-Bloch relation. Figure 2.9 is a plot of $\frac{d E}{d x}$ in the Drift Chamber as a function of tracks' momenta. 


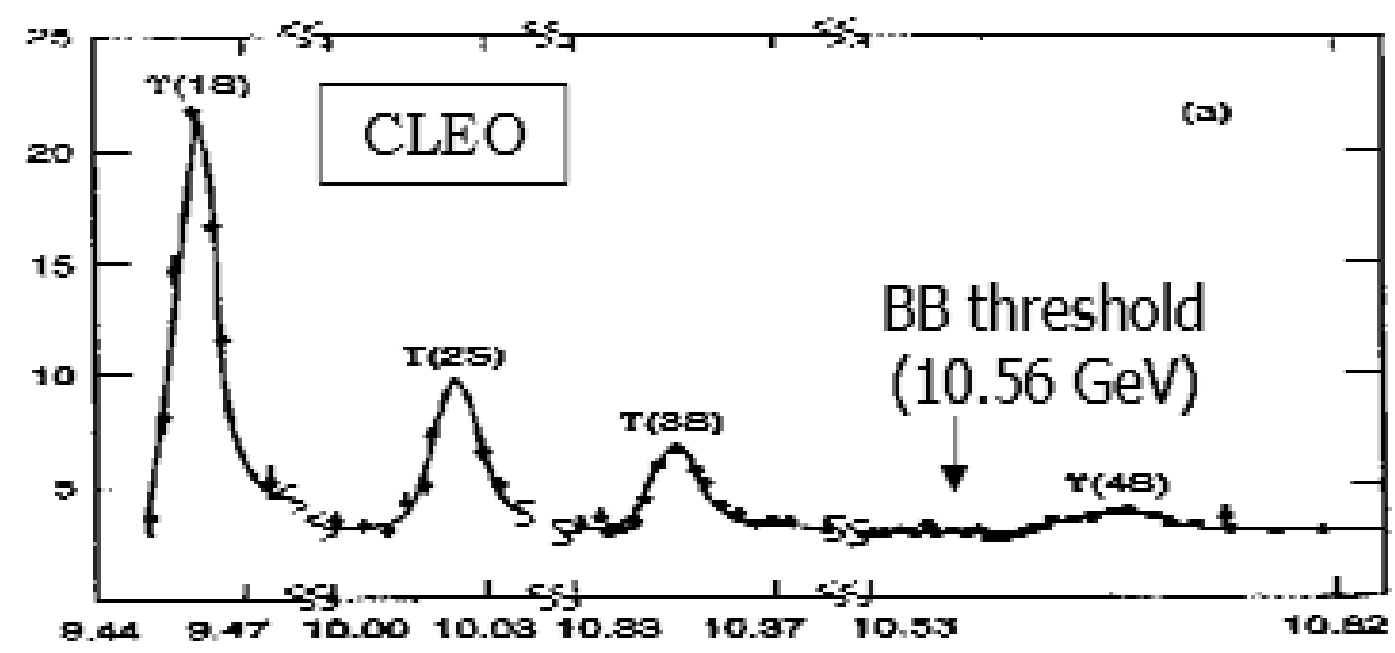

Figure 2.5: $\Upsilon$ Spectrum.

\subsection{Detector of Internally Reflected Cerenkov Light (DIRC)}

The Detector of Internally Reflected Cerenkov Light (DIRC) [31] is a sub-detector that distinguishes the different charged particle species. For example, the DIRC can distinguish between pions and kaons. Charged particles traveling through a dielectric media radiate photons on a conical wavefront. This is similar to a shock wave of a sonic boom (Figure 2.10). The opening angle for the cone is related to the index of refraction of the media and particle velocity by the relation: 


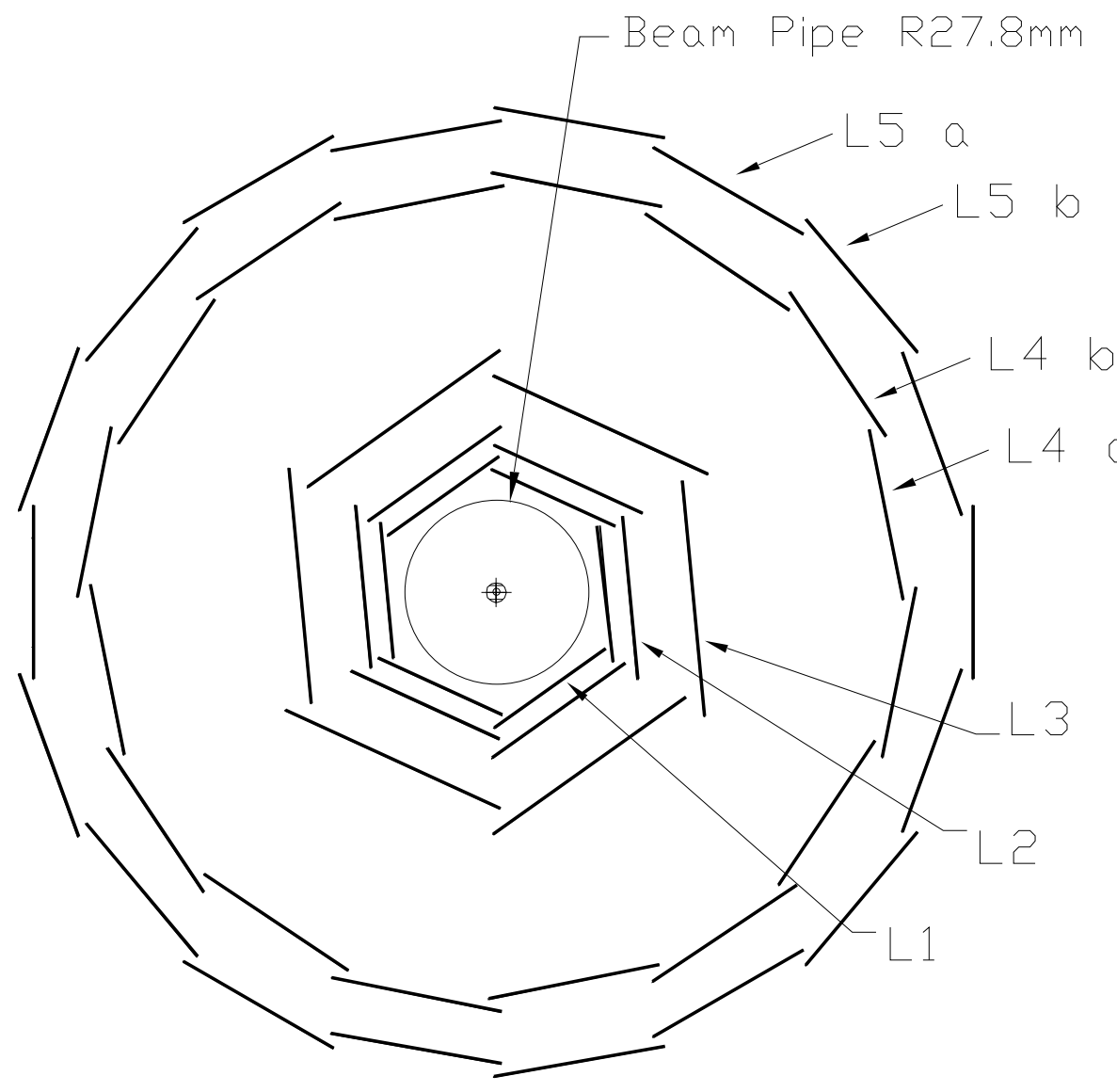

Figure 2.6: End view of the SVT.

$$
\cos \theta=\frac{1}{\beta n}
$$

where $\theta$ is the opening angle between the track and the cone (Figure 2.10) and: 


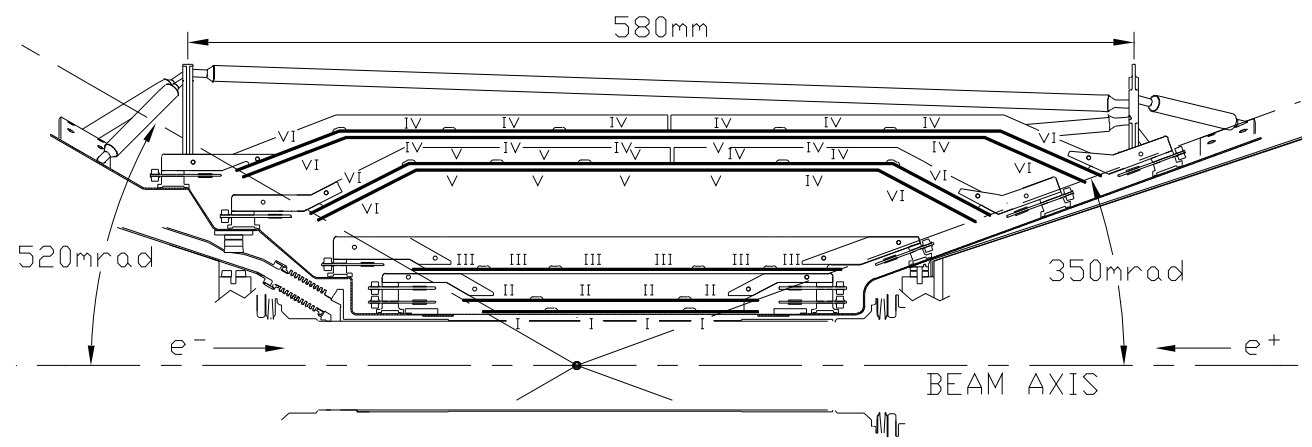

Figure 2.7: Side view of the SVT.

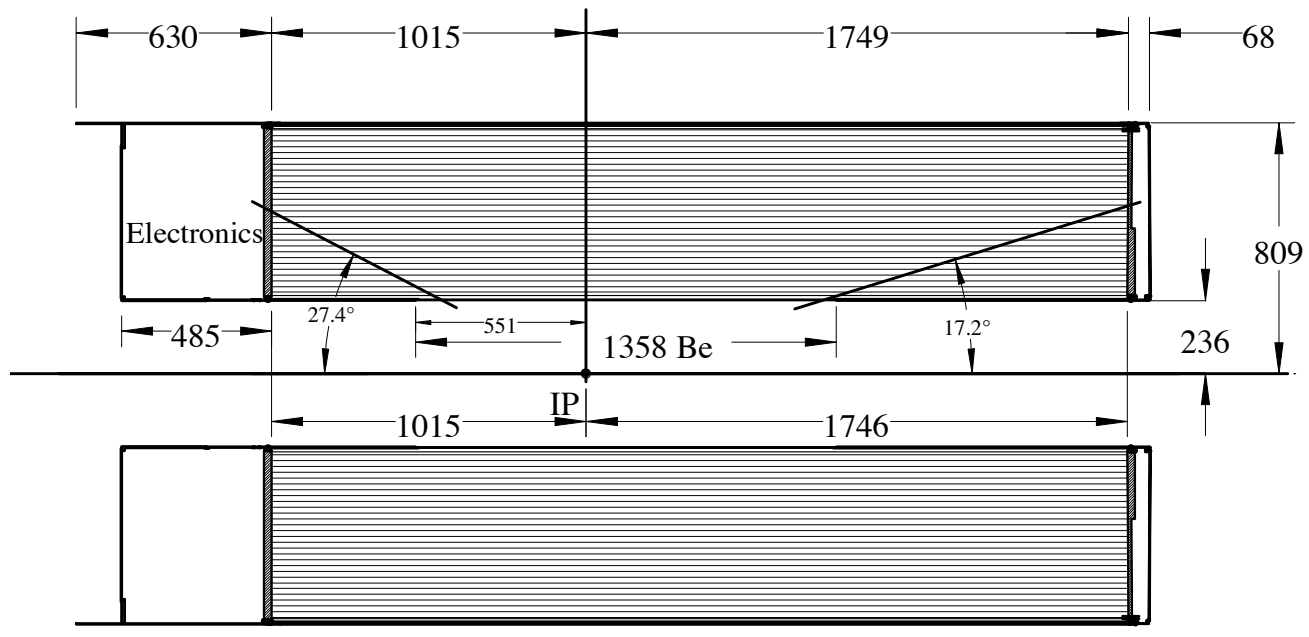

\section{BaBar Drift Chamber}

Figure 2.8: Longitudinal section of the Drift Chamber.

$$
\beta=\frac{v}{c}
$$

Timing information from the tracks in this sub-detector is recorded be- 


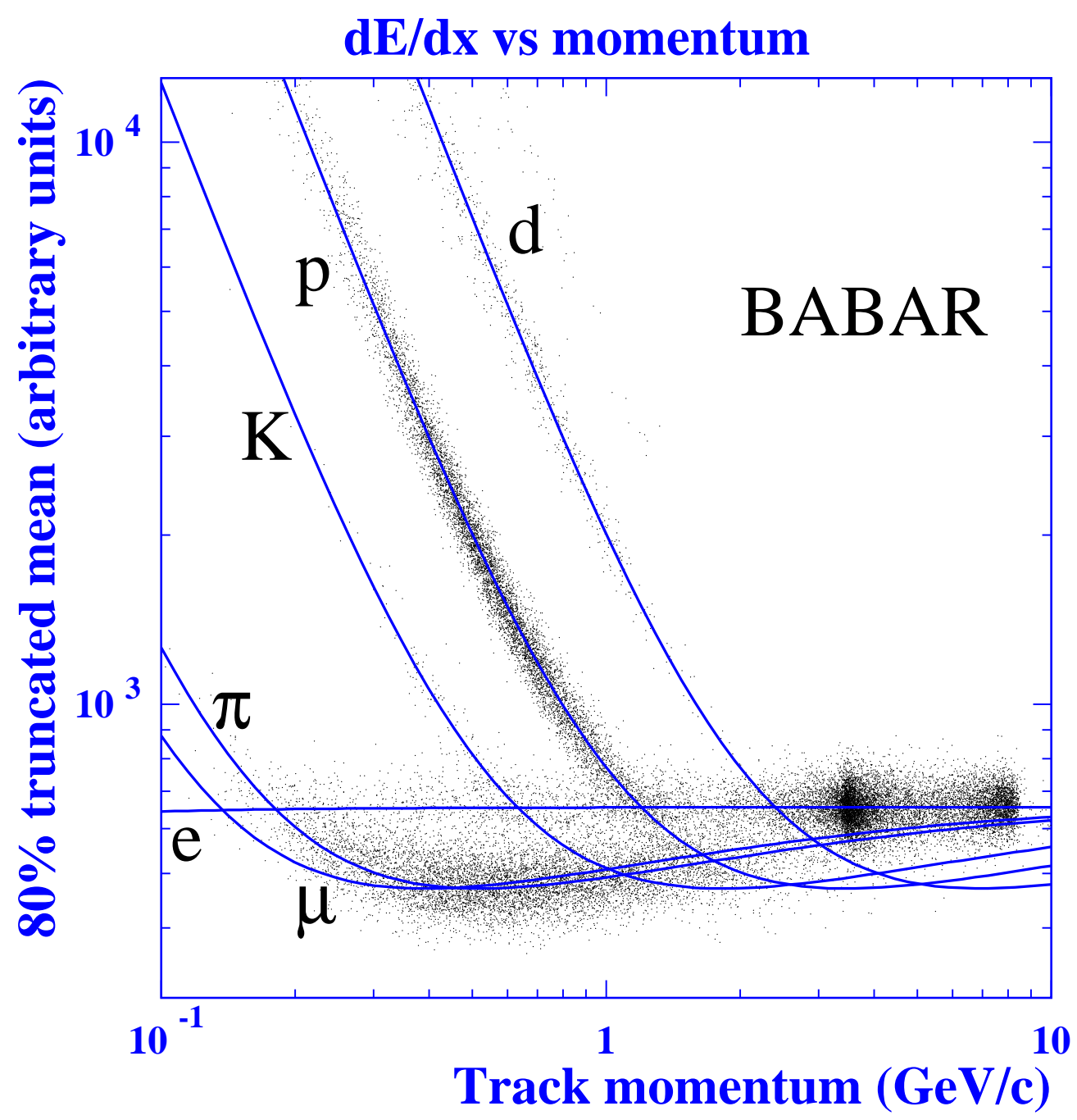

Figure 2.9: Measurement of $\frac{d E}{d x}$ in the Drift Chamber as a function of track momentum.

cause it contributes to background hit rejection and the separation of hits from differing tracks. The timing also gives information on the photon propagation angles. This gives BABAR an independent measurement of the Cerenkov angle. If the momentum is known via the SVT and the DCH and the velocity is 
known via the DIRC, the mass can be inferred.

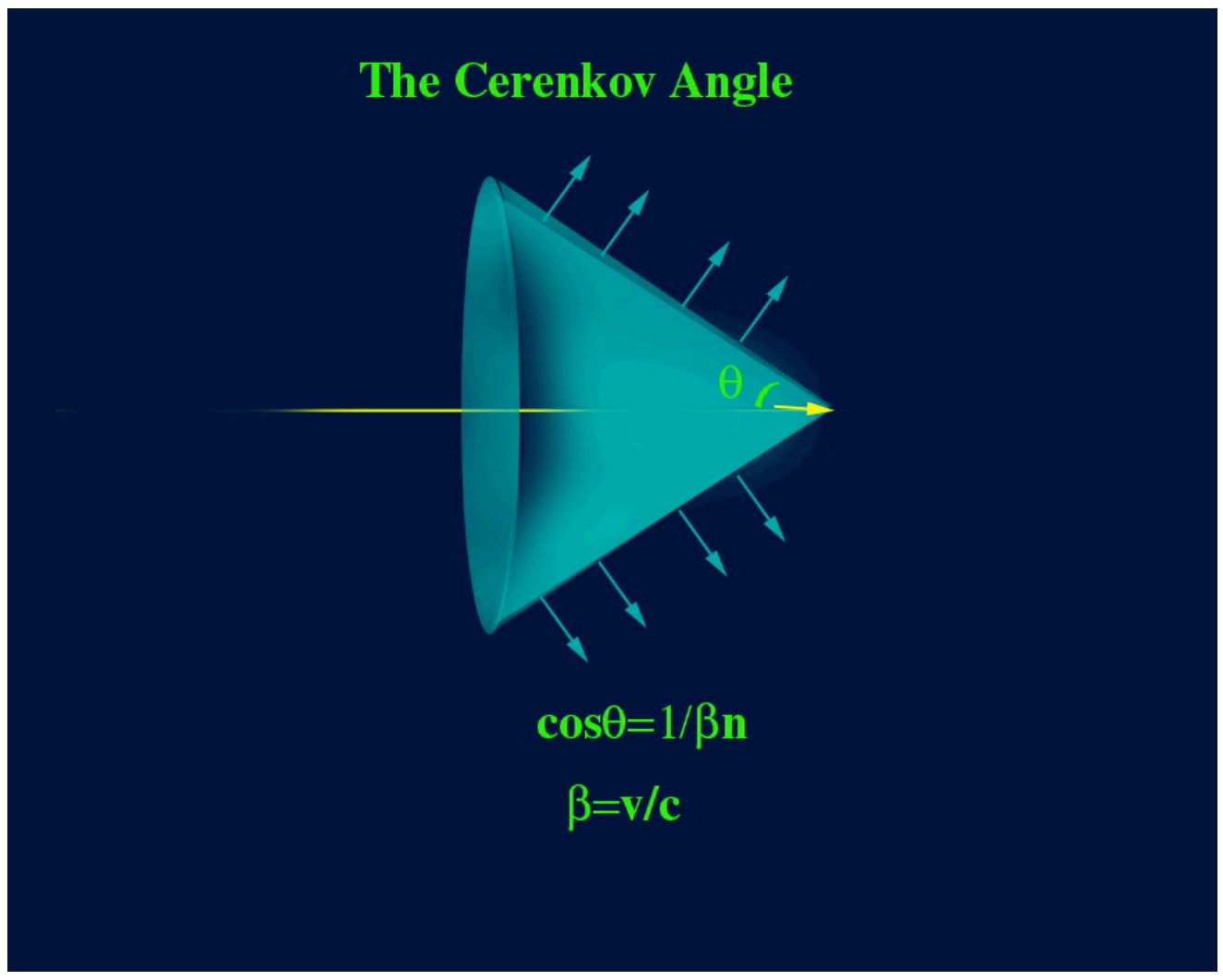

Figure 2.10: Cerenkov Angle.

\subsection{Electromagnetic Calorimeter (EMC)}

The Electromagnetic Calorimeter (EMC) [32, 33, 34] sub-detector as shown in Figures 2.11 and 2.12 is designed to detect photons and to help to identify electrons. For this analysis, the EMC is used to reconstruct $\pi^{0}$ candidates that decay into a pair of photons. They cascade by the processes of 
pair production and bremsstrahlung within the thallium doped cesium iodide crystals. The total ionization charge produced is proportional to the energy deposited in the calorimeter. The ionization produces scintillation light that is collected by a pair of photo diodes.

The EMC cesium iodide crystals vary in length from 29.6 to $32.4 \mathrm{~cm}$ and typically have a front face dimension of $4.7 \times 4.7 \mathrm{~cm}$. The crystals are mounted in carbon-fiber composite housings. These housings are then mounted on an aluminum frame. On the larger rear face of the crystal, two silicon PIN diodes are attached for readout.

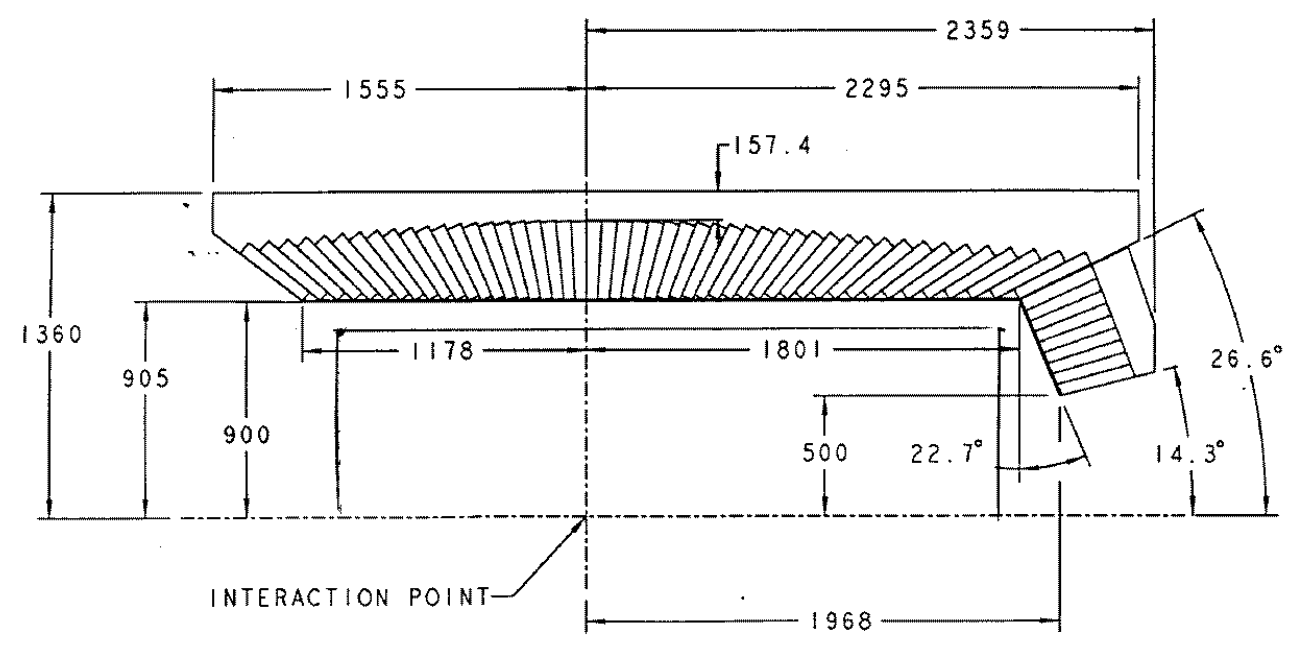

Figure 2.11: Longitudinal section of the EMC. 


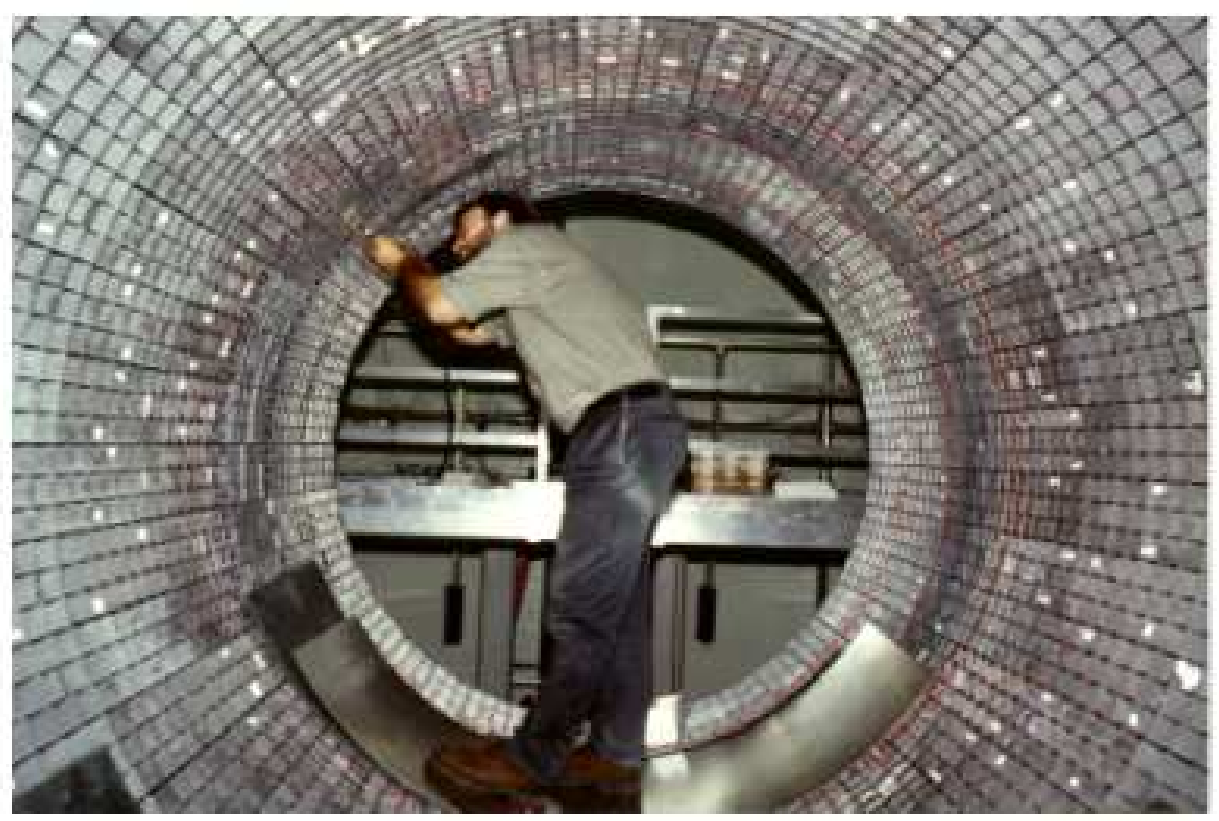

Figure 2.12: Electromagnetic Calorimeter Interior.

\subsection{Instrumented Flux Return (IFR)}

The Instrumented Flux Return (IFR) (Figure 2.13) detects muons [36, 37]. The IFR was built with layers of Resistive Plate Chambers (RPCs) and steel plates. The steel plates are there to separate pions and kaons from muons. The IFR also detects the position of neutral kaons $\left(K_{L}^{0}\right)$. The IFR consists of a barrel and end cap comprised of planar layers of RPC's external to the superconducting magnetic coil cryostat, and two layers of cylindrical RPC's between the EMC and the cryostat. Ionizing particles which cross the gap create tracks of ions and electrons in a gas mixture. This gas mixture is $56.7 \%$ 
Argon, $38.8 \%$ Freon, and $4.5 \%$ isobutane. Since this analysis does not use muons, information from this subdetector was not used.
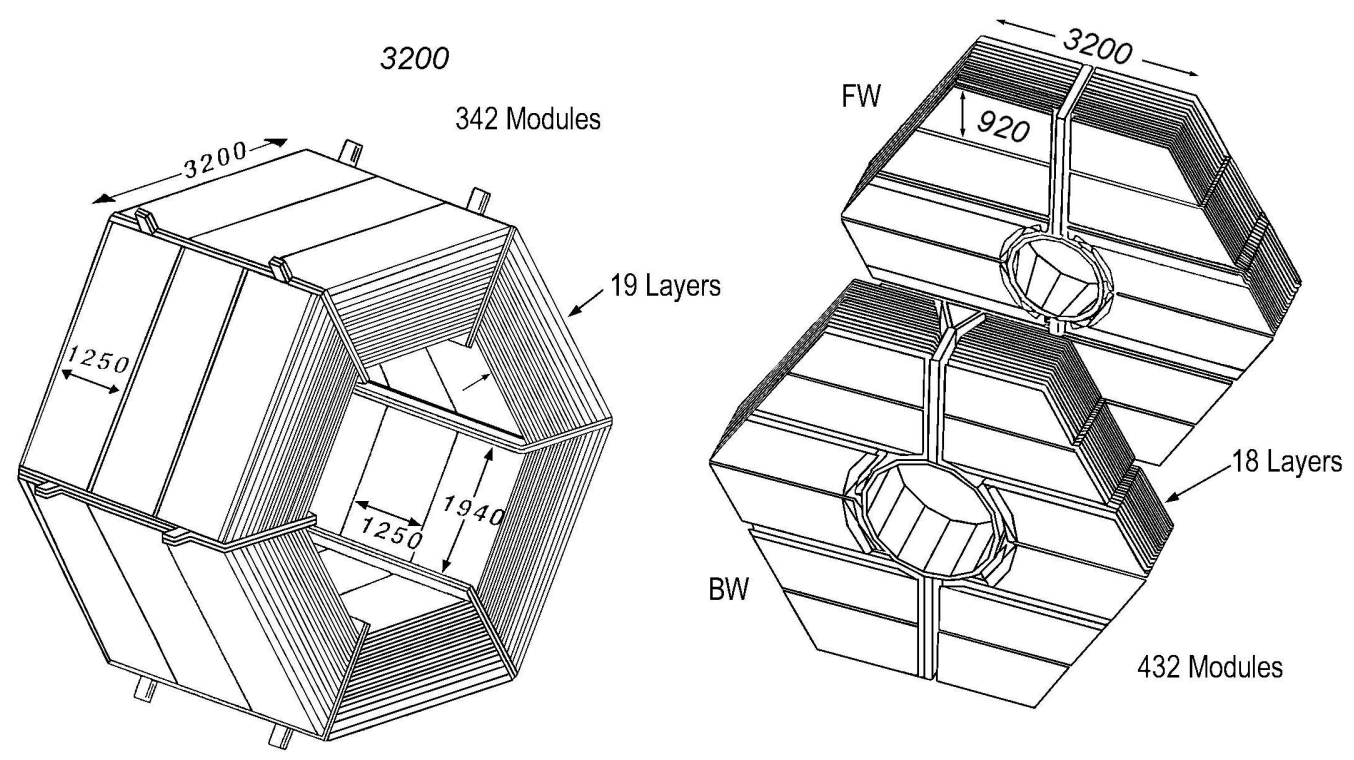

Figure 2.13: Instrumented Flux Return Section.

\subsection{Vertexing and Fitting}

With every event, a dozen or so tracks are recorded. Vertexing finds the most likely location of the decay vertices by finding the intersection point of the tracks of the prospective daughter particles. The procedure minimizes the sums of squares of the DOCA between tracks and vertex. Mass constraints can be used to improve the fit. In addition to the position, uncertainties are also determined. The chi-square for the vertex fit can be used as a cut parameter. 


\subsection{Trigger System}

The Trigger has a Level 1 (L1) and a Level 3 (L3). The L1 trigger is a hardware system that is responsible for the initial decision to pass along the event to the L3 software trigger for processing. The dominant source of events rejected by L1 is beam-gas or beam-wall interaction background, as well as cosmic rays. These can be separated from physics events by discriminating on the distance of the point of closest approach of the tracks to the IP. The trigger system must select events efficiently to keep the total event rate under $120 \mathrm{~Hz}$ and the background conditions low. 


\section{Chapter 3}

\section{Theoretical Background of $D_{J}^{0}$}

\subsection{Introduction}

In the study of the natural world, there are four fundamental forces that interact with the main components of the universe (Table 3.1). The Standard Model (SM) $[52,53,51]$ is a set of theories that try to predict the behavior of subatomic-particles using these forces except for gravity. Gravity is not considered in the standard model because its strength is very small when compared to the other three forces and because no one knows how to include it. The SM contains two sets of particles, fermions and bosons. Fermions are particles which obey the Pauli exclusion principle and have half integer spin. Bosons do not follow the Pauli exclusion principle and have an integer spin. 


\begin{tabular}{|cc|}
\hline Force & Strength \\
\hline Strong & 10 \\
Electromagnetic & $10^{-2}$ \\
Weak & $10^{-13}$ \\
Gravitational & $10^{-42}$ \\
\hline
\end{tabular}

Table 3.1: The four forces and their relative strengths.

The particles that transmit forces are bosons. And the common particles of matter are fermions.

The bosons, the particles that mediate the forces, of the SM are:

1. Photons (electromagnetic interaction)

2. $W^{ \pm}$and $Z^{0}$ bosons (weak interaction) $[54,56,57,58,59]$

3. Gluons (strong interaction).

The SM also predicts the existence of the Higgs particle [48, 49, 50], also called the standard model Higgs. However, the mass of the standard model Higgs is a free parameter. LEP experiments have measured the lower limit of the standard model Higgs to be larger than $114 \mathrm{GeV} / c^{2}$ at the $95 \%$ C.L. [47].

The fermions can be categorized into three generations. Ordinary matter is made of quarks (See Table 1.1), leptons (Figure 3.1), mesons (Figure 3.2) and baryons. Quarks consist of three generations: 


$$
(:)(:)(:)
$$

And leptons consist of three generations:

$$
\left(\begin{array}{c}
e \\
\nu_{e}
\end{array}\right)\left(\begin{array}{c}
\mu \\
\nu_{\mu}
\end{array}\right)\left(\begin{array}{c}
\tau \\
\nu_{\tau}
\end{array}\right)
$$

\begin{tabular}{|c|c|c|}
\hline \multicolumn{3}{|c|}{ Leptons spin $=1 / 2$} \\
\hline Flavor & $\begin{array}{l}\text { Mass } \\
\mathrm{GeV} / \mathrm{c}^{2}\end{array}$ & $\begin{array}{l}\text { Electric } \\
\text { charge }\end{array}$ \\
\hline 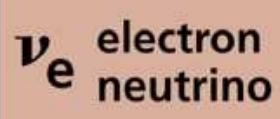 & $<1 \times 10^{-8}$ & 0 \\
\hline e electron & 0.000511 & -1 \\
\hline$v_{\mu} \begin{array}{c}\text { muon } \\
\text { neutrino }\end{array}$ & $<0.0002$ & 0 \\
\hline $\boldsymbol{\mu}$ muon & 0.106 & -1 \\
\hline $\boldsymbol{\nu}_{\boldsymbol{\tau}} \underset{\text { neutrino }}{\text { tau }}$ & $<0.02$ & 0 \\
\hline$\tau$ tau & 1.7771 & -1 \\
\hline
\end{tabular}

Figure 3.1: Lepton family [55]. 
The quarks listed in the previous tables can combine to form various particles. When three quarks combine, a baryon is formed. A bound state of a quark and an anti-quark is labeled a meson. All of the quarks have mass and fractional charge while leptons either have mass and unit charge or no charge and tiny masses.

\begin{tabular}{l|l|c|c|c|l} 
Symbol & Name & $\begin{array}{c}\text { Quark } \\
\text { content }\end{array}$ & $\begin{array}{c}\text { Electric } \\
\text { charge }\end{array}$ & $\begin{array}{c}\text { Mass } \\
\text { GeV/c }\end{array}$ & Spin \\
\hline $\boldsymbol{\pi}^{+}$ & pion & $\mathbf{u} \overline{\mathbf{d}}$ & +1 & 0.140 & 0 \\
$\mathbf{K}^{-}$ & kaon & $\mathbf{s} \overline{\mathbf{u}}$ & -1 & 0.494 & 0 \\
$\boldsymbol{\rho}^{+}$ & rho & $\begin{array}{r}\mathbf{u d} \\
\mathbf{d} \overline{\mathbf{b}}\end{array}$ & +1 & 0.770 & 1 \\
$\mathbf{B}^{\mathbf{0}}$ & B-zero & $\begin{array}{c}\mathbf{d} \\
\mathbf{C} \overline{\mathbf{C}}\end{array}$ & 0 & 2.980 & 0 \\
$\boldsymbol{\eta}_{\mathbf{C}}$ & eta-c &
\end{tabular}

Figure 3.2: Common Mesons [55].

The SM predicts attributes of the natural world. Theorists and experimentalist test this theory. But some fundamental properties have insufficient explanation. The SM unifies the electromagnetic and weak forces and makes predictions about the strong interaction.

After extensive testing for years, the predictive capabilities of the SM has proved to be very good. The only exception is the prediction of neutrino masses. 
To better test the SM, one must sensitively test the model for selfconsistency. This might include the exhaustive study of quark couplings in the weak interaction or the study of how the strong interaction determines the spectra and decays of the observed bound states of quarks.

\subsection{Heavy Quark Effective Theory}

The measurement of the $D_{J}^{0}$ properties, such as the decay widths and branching fractions of these particles, are important validations of Heavy Quark Effective Theory (HQET). As described in Chapter 1, HQET is an effective theory which assumes Heavy Quark Symmetry (HQS). This assumption is that the charm quark is much heavier than the other quark in the meson $\left(m_{c} \gg \Lambda_{Q C D}\right)[15,16]$

With this assumption, the $D_{J}^{0}$ mesons resemble the hydrogen atom with regard to their angular momentum coupling. The charm quark's spin $S_{c}$ separates from L, the orbital angular momentum as shown in Figure 1.1. The sum $j=L+S_{q}$, total angular momentum of the light quark degrees of freedom $\left(j_{q}\right.$ in Figure 1.1), is then conserved as a good quantum number. As a result, there are two sets of doublets. One with $j=\frac{3}{2}$ and a second one with $j=\frac{1}{2}$. 


\subsection{Introduction to the $D_{J}^{0}$}

The first observations of the $D_{J}^{0}$ excited charmed mesons were made by the ARGUS Collaboration [18, 4, 5], CLEO [6] and the Tagged Photon Spectrometer [19]. The notation $D_{J}\left(D^{* *}\right)$ refers to orbitally excited $D$ mesons, consisting of a charm quark and an up or down anti-quark with an orbital angular momentum of $L=1$ (P-wave).

The four neutral $D_{J}^{0}$ mesons are $D_{0}^{*}\left(j=\frac{1}{2}\right)^{0}, D_{1}\left(j=\frac{1}{2}\right)^{0}, D_{1}(2420)^{0}$, and $D_{2}^{*}(2460)^{0}$ (Figure 1.2 and Table 1.2). The two $D_{J}^{0}$ with $j=\frac{1}{2}$ are broad resonances (with a width of a few hundred $\mathrm{MeV}$ ) while the other two states are narrow resonances (width $20-40 \mathrm{MeV}$ ) [76]. Feynman diagrams of $D_{J}^{0}$ creation are shown in Figure 3.3.

In HQET, the $D_{J}^{0}$ states are built up by combining the heavy quark spin $S_{c}=\frac{1}{2}$ with $\mathrm{j}$, the angular momentum of the the light quark. For $\mathrm{L}=1$ :

$$
S_{c} \oplus j=1 \oplus \frac{1}{2}=\frac{3}{2}, \frac{1}{2} .
$$

For $j=\frac{1}{2}$, combining with the heavy quark spin one gets:

$$
S_{c} \oplus j=\frac{1}{2} \oplus \frac{1}{2}=0,1
$$

These states $(J=0, J=1)$ correspond to $D_{1}\left(j=\frac{1}{2}\right)^{0}, D_{0}^{*}\left(j=\frac{1}{2}\right)^{0}$. Combining 
$j=\frac{3}{2}$ with $S_{c}=\frac{1}{2}$ gives $D_{2}^{*}(2460)^{0}, D_{1}(2420)^{0}$ as:

$$
S_{c} \oplus j=\frac{1}{2} \oplus \frac{3}{2}=2,1
$$

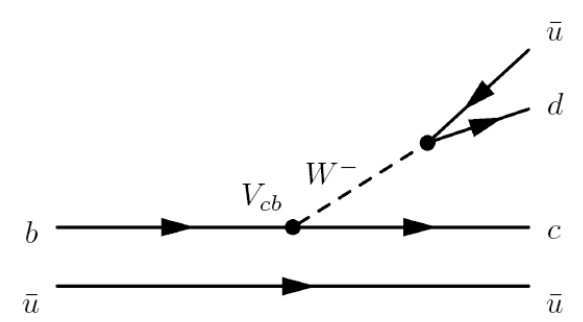

(a)

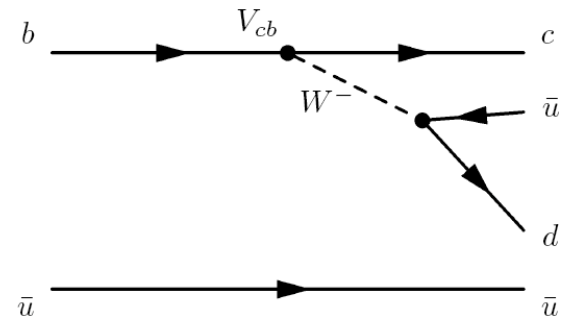

(b)

Figure 3.3: Feynman Diagrams of $D_{J}^{0}$ creation.

\section{$3.4 \quad D_{J}^{0}$ Allowed Decay Example}

Three conservation principles restrict the possible decay modes of the $D_{J}^{0}$ mesons:

1. Total angular momentum J

2. Parity

3. Angular momentum $\mathrm{j}$ of the light quark (an HQET requirement). 
As an example, consider the $D_{1}(2420)^{0}$. The $D_{1}(2420)^{0}$ has positive parity, $j=\frac{3}{2}$, and $J=1$. Two possible decay modes are considered: $D^{*+} \pi^{-}$ and $D^{+} \pi^{-}$. For $D \pi$, the total spin of this system is 0 . (Both the spin of $\mathrm{D}$ and $\pi$ are $0 . S=0+0=0$ ) For this decay to be possible, one of the requirements it must satisfy is $J_{f}=L \oplus S_{f}$ where the total angular momentum, $J_{f}$, equals 1. The various solutions are considered:

1. $\mathrm{L}=0$ fails. (s-wave decay) $0 \oplus 0 \neq 1$

2. L $=1$ satisfies $J_{f}$. (p-wave decay) $1 \oplus 0=1$

3. $\mathrm{L}=2$ fails. (d-wave decay) $2 \oplus 0 \neq 1$

So the p-wave decay is possible for $D_{1}(2420)^{0} \rightarrow D^{+} \pi^{-}$. Next, the p-wave is checked for parity:

$$
\Pi_{f}=\left(\Pi_{D^{*}}\right)\left(\Pi_{\pi}\right)(-1)^{L}=(-1)(-1)(-1)^{1}=-1 \neq \Pi_{D_{1}(2420)^{0}}=1
$$

and $D_{1}(2420)^{0} \rightarrow D \pi$ is not possible.

For $D^{*} \pi$, the total spin of this system is 1 . The spin of the $D^{*+}$ is 1 and the pion's spin is $0, S=1+0=1$. For the L conservation check:

1. $\mathrm{L}=0$ satisfies $J_{f} .0 \oplus 1=1,0$ 
2. $\mathrm{L}=1$ satisfies $J_{f} .1 \oplus 1=2,1,0$

3. $\mathrm{L}=2$ satisfies $J_{f} \cdot 2 \oplus 1=3,2,1$

So all three decay modes satisfy L for $D_{1}(2420)^{0} \rightarrow D^{*+} \pi^{-}$.

But when the final parity is measured:

1. $\mathrm{L}=0$ works. $(-1)(-1)(-1)^{0}=1=\Pi_{D_{1}(2420)^{0}}$

2. $\mathrm{L}=1$ fails. $(-1)(-1)(-1)^{1}=-1 \neq \Pi_{D_{1}(2420)^{0}}=1$

3. $\mathrm{L}=2$ works. $(-1)(-1)(-1)^{2}=1=\Pi_{D_{1}(2420)^{0}}$

So only the s-wave and d-wave decays remain. But for $\mathrm{L}=0$, the s-wave decay

fails because $j_{q}$ is not conserved $\left(j_{i}=\frac{3}{2}\right.$ and $\left.j_{f}=\frac{1}{2}\right)$. But $j_{q}$ is conserved for $L=2$. Therefore, for $D_{1}(2420)^{0}$ will only go to $D^{*+} \pi^{-}$via a $\mathrm{D}$-wave decay. A similar procedure is used for the other three particles of the $D_{J}^{0}$ and explains why the particles decay in specific ways.

\subsection{Narrow and Wide Resonances}

The decay amplitudes of the $D_{J}^{0}$ are suppressed by a factor of pion momentum to a power of L [78]. Higher L values suppress decay rates. The lower the decay rate, the narrower the resonance in keeping with the uncertainty principle. And this is the reason that the $D_{2}^{*}(2460)^{0}$ and the $D_{1}(2420)^{0}$ which 
decay by the the higher $\mathrm{L}$ d-wave decays are the narrow resonances, while $D_{1}\left(j=\frac{1}{2}\right)^{0}$ and $D_{0}^{*}\left(j=\frac{1}{2}\right)^{0}$ which decay by the lower L s-wave decays are the wide resonances.

With a better understanding of the $D_{J}^{0}$, a greater understanding of HQET is achieved. And with a better understanding of HQET, progress in $\mathrm{CP}$ violation and the CKM matrix may be reached. 


\section{Chapter 4}

\section{Analysis}

\subsection{Introduction}

The previous two chapters describe the experiment that observes the $D_{J}^{0}$ spectroscopy and the fundamental theories that govern the family's behavior. This chapter details:

1. Data acquisition.

2. Measuring various experimental values used for event selection.

3. Estimating various systematic uncertainties. 


\subsection{Data Acquisition}

The data set for $B A B A R$ is large and on the order of hundreds of terabytes. When BABAR was proposed in 1993, a similarly sized data set had just been successfully recorded and reconstructed for the first time at Fermilab E791 [41, 42, 43]. Information digitized by the BABAR detector covers everything from when it was recorded. To analyze [44, 45] all of this is inefficient. Most of the events collected are not even relevant to this analysis. Most events are just $e^{+} e^{-} \rightarrow e^{+} e^{-}$interactions. From a data rate of $1500 \mathrm{~Hz}$, only the $4 \mathrm{~Hz}$ of $\Upsilon(4 S)$ events are recorded, plus a bit of calibration data [24]. So a series of steps for many analyses was developed:

1. Skimming

Millions of $B \bar{B}$ events are initially studied and reduced to a more manageable number through the application of few event 'requirements'.

2. Tupling

Event data are condensed into a data file of tuples that are analyzed by the Root program that is described in a later section in this chapter.

\section{Optimization}

Optimization is the stage where event variable cut values are determined to minimize the uncertainty on the branching fraction. 


\section{Counting and Fitting}

Yields that are used for the various branching fractions are measured. Fitting procedures are applied to determine masses. Systematic errors are estimated.

\subsection{Four Vectors - Particle Reconstruction}

As described in detail in Chapter 2, the event is processed by a variety of sub-detectors. The initial particles and the ones later reconstructed are all in the form of a 4 vector, $\mathbf{p}=(E, \vec{p})$, an energy of the particle and its momentum three-vector, where $E^{2}=|\vec{p}|^{2}+m^{2}$. It possesses a velocity $\beta=\mathrm{v} / \mathrm{c}$.

For a reconstruction of a parent from its $\mathrm{n}$ daughters:

$$
m^{2}=\left(\sum_{i=1}^{n} E_{i}\right)^{2}-\left|\sum_{i=1}^{n} \overrightarrow{p_{i}}\right|^{2}
$$

For a two body decay, Equation 4.1 becomes:

$$
E_{c m}=\sqrt{\left[m_{1}^{2}+m_{2}^{2}+2 E_{1} E_{2}\left(1-\beta_{1} \beta_{2} \cos \theta\right)\right]}
$$

Where $\theta$ is the lab frame angle between the two daughter particles.

These equations and the track fitting process are used in the reconstruction of the rest mass and the four momentum of the short lived decay particles. 
$\mathrm{dE} / \mathrm{dx}$ loss and the Cerenkov counter are used to identify the species of the particle to supply the masses of the daughters for use in the reconstruction.

\subsection{Base Modes}

The quantities that are the subject of this analysis are measured through the reconstruction of the $B^{-}$meson through the states, $D^{*+} \pi^{-} \pi^{-}$and $D^{+} \pi^{-} \pi^{-}$. Particles of the $D_{J}^{0}$ group are contained within these sets. The remaining candidates consist of the non-resonant modes, $B^{-} \rightarrow D^{+} \pi^{-} \pi^{-}(n r)$ and $B^{-} \rightarrow$ $D^{+*} \pi^{-} \pi^{-}(n r)$

$D^{*+}$ candidates are reconstructed with $D^{0}$ and $\pi^{+}$candidates. $D^{0}$ candidates are reconstructed using the following modes: $D^{0} \rightarrow K^{-} \pi^{+}, D^{0} \rightarrow$ $K^{-} \pi^{+} \pi^{0}$, and $D^{0} \rightarrow K^{-} \pi^{+} \pi^{-} \pi^{+} . D^{+}$candidates are reconstructed using the $D^{+} \rightarrow K^{-} \pi^{+} \pi^{+}$decay mode. The decay chain can be seen in Table 4.1. Backgrounds, events that are not the desired mode, but still potentially reconstruct into events, are also studied.

\begin{tabular}{|l|l|l|}
\hline Inclusive Mode & $B^{-} \rightarrow D^{+} \pi^{-} \pi^{-}$ & $B^{-} \rightarrow D^{*+} \pi^{-} \pi^{-}\left(D^{*+} \rightarrow D^{0} \pi^{+}\right)$ \\
\hline Base Modes & $D^{+} \rightarrow K^{-} \pi^{+} \pi^{+}$ & $D^{0} \rightarrow K^{-} \pi^{+}$ \\
& & $D^{0} \rightarrow K^{-} \pi^{+} \pi^{0}$ \\
& & $D^{0} \rightarrow K^{-} \pi^{+} \pi^{-} \pi^{+}$ \\
\hline
\end{tabular}

Table 4.1: Decay modes used for the analysis. 


\subsection{Root}

Root is a program that manages and analyzes data. It provides a framework for histogramming, fitting, and graphically representing data in a variety of formats either in batch mode or interactively. It manipulates data in the Root format. An ntuple is an object of data that contains a set of data objects. Mathematicians use the word ntuple to describe any ordered set of $\mathrm{N}$ objects. In Root, an ntuple may consist of any $\mathrm{N}$ objects belonging to arbitrary $\mathrm{C}++$ classes.

\subsection{Available Data}

The data for this analysis comes from Run 1 through Run 5 (Table 4.2). The data was collected over a six year period. Table 4.4 shows the periods of times that each run collected data. A run may be defined as the period in which the detector collects data. Runs are typically separated from each other by a maintenance period of a few weeks to a few months. The processed data is stored in the Root file format. An analysis routine processes the data into another Root file containing reconstructed particles information. Root handles all of the analysis from this stage forward. 


\begin{tabular}{|c|c|c|}
\hline Type of data & Number of events & Luminosity $\left(\mathrm{fb}^{-1}\right)$ \\
\hline$\Upsilon(4 S)$ data & 382.92 million $B \bar{B}$ & 343.38 \\
Off- $(4 S)$ data & 44.9 million $q \bar{q}$ & 36.18 \\
\hline$B^{+} B^{-}$generic MC & 555 million & 1023 \\
$B^{0} \bar{B}^{0}$ generic MC & 552 million & 1018 \\
\hline
\end{tabular}

Table 4.2: Overview of data and generic Monte Carlo samples used in the analysis.

\subsection{Monte Carlo (MC) Samples}

In addition to the analysis of real data, additional studies are performed on the available MC data. Monte Carlo Event Data are 'generated' by the MC Generation Analysis Working Group (AWG) group. MC data is used to develop and test the analysis program and simulate the on-resonance $\Upsilon(4 S)$ decays as well as off-resonance. This MC sample is used for the determination of the reconstruction efficiencies of the particle reconstruction, the background studies and the systematic uncertainties. It is also used for the optimization of selection criteria. The MC samples are subjected to the standard event reconstruction. The various detector errors and beam background effects are included.

There exist a variety of available MC samples. They fall into two categories, Generic MC and Signal MC. Generic MC consists of the collected repertoire of known modes. Signal MC are sets which contain specific modes. Both kinds of MC samples include a complete detector response in order to 


\begin{tabular}{|l|c|}
\hline Sample & $\begin{array}{c}\text { \# events } \\
\text { (in units of 1000) }\end{array}$ \\
\hline$B^{-} \rightarrow D_{1}(j=1 / 2) \pi, D^{0} \rightarrow 4$ modes & 350 \\
\hline$B^{-} \rightarrow D_{1}(2420) \pi, D^{0} \rightarrow 4$ modes & 350 \\
\hline$B^{-} \rightarrow D_{2}^{*}(2460) \pi, D^{0} \rightarrow 4$ modes & 348 \\
\hline$B^{-} \rightarrow D^{*+} \pi^{-} \pi^{-}$(non-resonant) & \\
$D^{0} \rightarrow K \pi$ & 704 \\
$B^{-} \rightarrow D^{*+} \pi^{-} \pi^{-}$(non-resonant) & \\
$D^{0} \rightarrow 4$ modes & 704 \\
\hline$B^{-} \rightarrow D_{0}^{*}(j=1 / 2) \pi, D_{0}^{*} \rightarrow D^{+} \pi^{-}, D^{+} \rightarrow K \pi \pi$ & 818 \\
\hline$B^{-} \rightarrow D_{2}^{*}(2460) \pi, D_{2}^{*} \rightarrow D^{+} \pi^{-}, D^{+} \rightarrow K \pi \pi$ & 350 \\
\hline$B^{-} \rightarrow D^{+} \pi^{-} \pi^{-}($non-resonant) & \\
$D^{+} \rightarrow K \pi \pi$ & 714 \\
$B^{-} \rightarrow D^{+} \pi^{-} \pi^{-}($non-resonant) & 704 \\
$D^{+} \rightarrow K \pi \pi, K_{S} \pi$ & \\
\hline
\end{tabular}

Table 4.3: Table of non-generic Monte Carlo samples used in the analysis.

make the simulated events match with the data. Tables 4.2 and 4.3 list the used Generic MC and Signal MC data sets.

MC data is simulated using GEANT, a program that considers the behavior of the detector (background, electronic circuits, etc.). Generator level processes produce long lived particles according to known physical cross sections and other particle properties. The detector response is simulated by calculating localized ionization deposits. Finally, the response of the electronics to ionization energy depositions is simulated to produce digitizations. Energy contributions from randomly triggered events are also added into the data to represent beam backgrounds. 


\begin{tabular}{|c|c|c|}
\hline Run & Start & End \\
\hline 1 & February, 2000 & November, 2000 \\
2 & February, 2001 & July, 2002 \\
3 & December, 2002 & June, 2003 \\
4 & September, 2004 & August, 2004 \\
5 & December, 2004 & July, 2006 \\
\hline
\end{tabular}

Table 4.4: Data collection dates

\subsection{Event Reconstruction and Selection}

From a list of labeled tracks, $B^{ \pm}$mesons are reconstructed. The reconstruction routines labels tracks with certain identifications. Among these are:

1. GoodTracksVeryLoose (GTVL)

2. GoodTracksLoose (GTL)

3. KLHNotPion

4. KLHTight

GoodTracksVeryLoose may be applied to a particle track with the following characteristics:

1. Maximum Momentum: $10 \mathrm{GeV} / \mathrm{c}$ 
2. Maximum DOCA in XY plane: $1.5 \mathrm{~cm}$

3. Maximum Absolute Value of Z DOCA: $10 \mathrm{~cm}$

GTL is a subset of GoodTracksVeryLoose but has the additional requirements:

1. Minimum Transverse Momentum: $0.1 \mathrm{GeV} / \mathrm{c}$

2. Minimum number of hits in the drift chamber: 12

KLHNotPion and KLHTight are labels for particles that may be Kaons. Kaons that satisfy:

$$
L K /(L K+L P i)>0.20
$$

or

$$
\text { LPro } /(\text { LPro }+L P i)>0.20
$$

can be KLHNotPion but if the likelihood equations add up to:

$$
L K /(L K+L P i)>0.90
$$

and 


$$
L K /(L P r o+L K)>0.20
$$

then the particle can be labeled as KLHTight. LK is the likelihood that the particle is a kaon. LPi is the likelihood the particle is a pion. And LPro is the likelihood of it being a proton. These values are found from likelihoods determined from the SVT, DCH and DIRC subsystems. The SVT and DCH use the particles $\frac{d E}{d x}$. The DIRC uses the particle's track quality, its Cerenkov angle, the last layer of the $\mathrm{DCH}$ to see the hit and the energy seen by the calorimeter. These values are compared with the values determined from MC studies to determine particle identity. For each of the reconstructed particles, cut selections are made based on the quality of the track.

\subsubsection{Charged Track Selection}

All pions used satisfy the GTL selection. This selection requires that pions have a minimum momentum of $100 \mathrm{MeV} / c$. The kaons used satisfy the KLHTight selection. Further detail is shown in various BABAR reference materials of the Particle Identification Work Group [60]. 


\subsection{2 $\pi^{0}$ Candidates}

For the mode $D^{0} \rightarrow K^{-} \pi^{+} \pi^{0}, \pi^{0}$ candidates are used. The photons used for $\pi^{0} \rightarrow \gamma \gamma$ must satisfy a minimum energy requirement (See Table 4.5). The $\pi^{0}$ created must be within an acceptable limit of the Particle Data Group (PDG) mass of that particle.

$$
\left|M_{m\left(\pi^{0}\right)}-M_{m\left(\pi^{0}\right) P D G}\right|<14.9 \mathrm{MeV} / c^{2}
$$

\subsection{3 $D^{0}$ Reconstruction}

$D^{0}$ candidates are constructed using the following three modes:

1. $D^{0} \rightarrow K^{-} \pi^{+}$

2. $D^{0} \rightarrow K^{-} \pi^{+} \pi^{0}$

3. $D^{0} \rightarrow K^{-} \pi^{+} \pi^{-} \pi^{+}$

All kaon tracks must satisfy the KLHNotPion selection. They must satisfy a mass cut and be within a certain range around the accepted PDG value for the mass of the $D^{0}$ mesons. The pions come from the list KLHTight.

For analysis, these $D^{0}$ 's must satisfy a selection criterion based upon their mass differences with the PDG value (depending on the mode): 


$$
\begin{gathered}
\left|M_{m\left(D^{0}\left(D^{0} \rightarrow K^{-} \pi^{+}\right)\right)}-M_{m\left(D^{0}\right) P D G}\right|<41 \mathrm{MeV} / c^{2} \\
\left|M_{m\left(D^{0}\left(D^{0} \rightarrow K^{-} \pi^{+} \pi^{0}\right)\right)}-M_{m\left(D^{0}\right) P D G}\right|<33 \mathrm{MeV} / c^{2} \\
\left|M_{m\left(D^{0}\left(D^{0} \rightarrow K^{-} \pi^{+} \pi^{-} \pi^{+}\right)\right)}-M_{m\left(D^{0}\right) P D G}\right|<17 \mathrm{MeV} / c^{2}
\end{gathered}
$$

\subsection{4 $D^{*+}$ Reconstruction}

The $D^{*+}$ candidates are reconstructed by combining $D^{0}$ candidates with

pions. A cut window around the mass difference peak as shown in Equations $4.11-4.13$ is applied. This selection is used during the cut optimization process. The pions again come from the GoodTracksVeryLoose list. The candidates must also satisfy the requirement:

$$
\left|\Delta\left(m\left(D^{0} \pi\right)-m\left(D^{0}\right)\right)_{D^{0} \rightarrow K^{-} \pi^{+}}-\Delta\left(m\left(D^{0} \pi\right)-m\left(D^{0}\right)\right)_{P D G}\right|<4.8 \mathrm{MeV} / c^{2}
$$




$$
\left|\Delta\left(m\left(D^{0} \pi\right)-m\left(D^{0}\right)\right)_{D^{0} \rightarrow K^{-} \pi^{+} \pi^{0}}-\Delta\left(m\left(D^{0} \pi\right)-m\left(D^{0}\right)\right)_{P D G}\right|<3.6 \mathrm{MeV} / c^{2}
$$

$$
\left|\Delta\left(m\left(D^{0} \pi\right)-m\left(D^{0}\right)\right)_{D^{0} \rightarrow K^{-} \pi^{+} \pi^{-} \pi^{+}}-\Delta\left(m\left(D^{0} \pi\right)-m\left(D^{0}\right)\right)_{P D G}\right|<3.1 \mathrm{MeV} / c^{2}
$$

\subsection{5 $D^{+}$Reconstruction}

$D^{+}$'s are reconstructed using the mode $D^{+} \rightarrow K^{-} \pi^{+} \pi^{+}$. Kaons used for this mode must satisfy the KLHTight selector. Once again the pions come from the GTL list. These $D^{+}$candidates must satisfy a mass difference requirement as:

$$
\left|M_{m\left(D^{+}\right)}-M_{m\left(D^{+}\right) P D G}\right|<11.3 \mathrm{MeV} / c^{2}
$$

\subsection{6 $\quad B^{ \pm}$Reconstruction}

$B^{\prime} s$ are constructed using two modes: $B^{-} \rightarrow D^{+} \pi^{-} \pi^{-}, B^{-} \rightarrow D^{*+} \pi^{-} \pi^{-}$.

The constructor combines a $D^{*}$ or a $D^{+}$with two pions. $\Delta E$ and $m_{\mathrm{ES}}$ are two 
complementary kinematic variables used to discriminate against background $B^{ \pm}$meson candidates. These are also uncorrelated variables that make maximal use of available information [62]. $m_{E S}$ is used as a cut parameter and the $\Delta E$ distributions are fitted to obtain the yields.

$\Delta E$ is defined as:

$$
\Delta E=\sum_{i} \sqrt{m_{i}^{2}+\left(\vec{p}_{i}^{*}\right)^{2}}-E_{\text {beam }}^{*}
$$

where:

1. $E_{\text {beam }}^{*}$ is the energy of the beam in the $\Upsilon(4 S)$ CM frame.

2. $\vec{p}_{i}^{*}$ is the center of mass momentum of particle i for the $\mathrm{B}$ candidate.

3. $m_{i}$ is the mass of particle $\mathrm{i}$.

The quantity $\Delta E$ is used as a measure of the consistency of the candidate system of particles total energy with the beam energy in the CM frame.

\subsection{Event Variables}

When the Root file is created, many variables are stored. There are between six $\left(B^{-} \rightarrow D^{+} \pi^{-} \pi^{-}\right)$and ten variables $\left(B^{-} \rightarrow D^{*+} \pi^{-} \pi^{-}\right)$that are described to cut on the event candidates. The list of variables and their cut 


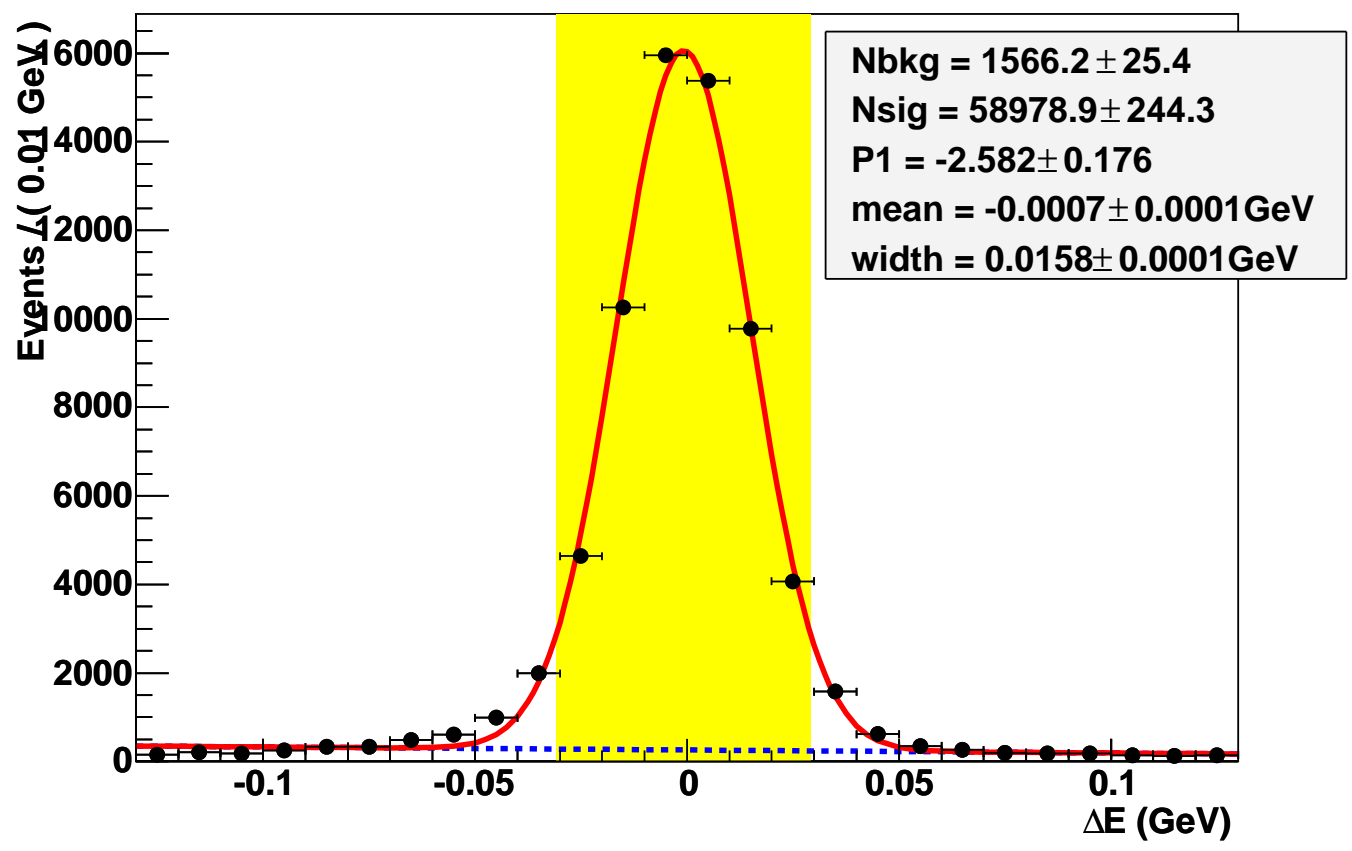

Figure 4.1: $D^{0} \rightarrow K^{-} \pi^{+}$Sample $\Delta E$ plot showing the Gaussian and linear components.

values are shown in Table (4.5) The values are the ones determined by the optimization routine used on page 57.

\subsubsection{B Candidate $m_{E S}$}

$m_{\mathrm{ES}}$ is the other property of the $B^{+}$and it is measured by:

$$
m_{\mathrm{ES}}=\sqrt{\left(E_{\mathrm{beam}}^{*}\right)^{2}-\left(\sum_{i} \vec{p}_{i}^{*}\right)^{2}}
$$

where $E_{\text {beam }}^{*}$ is the beam energy in the $\Upsilon(4 S) \mathrm{CM}$ frame and $\vec{p}_{i}^{*}$ is the CM 


\begin{tabular}{|l|ccc|c|}
\hline \multirow{2}{*}{ Cuts } & \multicolumn{3}{|c|}{$B^{-} \rightarrow D^{*+} \pi^{-} \pi^{-}$} & $B^{-} \rightarrow D^{+} \pi^{-} \pi^{-}$ \\
\hline$R_{2}$ & $D^{0} \rightarrow K^{-} \pi^{+}$ & $D^{0} \rightarrow K^{-} \pi^{+} \pi^{0}$ & $D^{0} \rightarrow K^{-} \pi^{+} \pi^{-} \pi^{+}$ & $D^{+} \rightarrow K^{-} \pi^{+} \pi^{+}$ \\
$\cos \theta_{\text {thrust }}$ & $<1.0$ & $<0.5$ & $<0.5$ & $<0.35$ \\
$m_{\mathrm{ES}}$ & $<1.0$ & $<1.0$ & $<1.0$ & $<0.9$ \\
$|\Delta E|$ & $>5.278$ & $>5.275$ & $>5.275$ & $>5.275$ \\
$\left|\Delta\left[m\left(D^{*+}\right)-m\left(D^{0}\right)\right]\right|$ & $<24$ & $<28$ & $<28$ & $<27$ \\
$\left|\Delta m\left(D^{0}\right)\right|$ & $<4.8$ & $<3.6$ & $<3.1$ & - \\
$\left|\Delta m\left(D^{+}\right)\right|$ & $<41$ & $<33$ & $<17$ & - \\
$\operatorname{Kaon}$ ID & - & - & - & $<11.3$ \\
$\left|\Delta m\left(\pi^{0}\right)\right|$ & Tight & Tight & Tight & - \\
$E_{\min }(\gamma)$ & - & $<14.9$ & - & - \\
Dalitz weight & - & $>35$ & - & - \\
\hline
\end{tabular}

Table 4.5: Table of cut variables' values.

momentum of particle $i$ in the candidate $B^{-}$-meson system.

For signal events, the beam-energy-substituted $B^{-}$mass, $m_{\mathrm{ES}}$, peaks at $m_{B}$. Figures 5.9 through 5.11 are the $m_{\mathrm{ES}}$ plots for this analysis. The value of the $m_{E S}$ cut depends on the decay mode as tabulated in Table 4.5.

\subsubsection{Event Shape}

Using values which characterize the shape of the event, one can remove a larger number of background events. One such variable is the Fox-Wolfram [79] value $R_{2}$. This is the ratio of the Fox-Wolfram second moment to the Fox-Wolfram zeroth moment.

The Fox Wolfram moment, $H_{l}$ is defined as: 


$$
H_{l}=\sum_{i, j} \frac{\left|p_{i}\right|\left|p_{j}\right|}{E_{v i s}^{2}} P_{l}\left(\cos \theta_{i j}\right)
$$

and $R_{2}$ is defined as:

$$
R_{2} \equiv \frac{H_{2}}{H_{0}}
$$

Where:

1. $P_{l}$ are the Legendre polynomials.

2. $p_{i, j}$ are the particles' momenta.

3. $\theta_{i j}$ is the opening angle between particles $i$ and $j$.

4. $E_{v i s}$ is the total energy for the event.

The value of $R_{2}$ ranges over $(0,1)$. Values closer to 0 indicates a more spherical event (typical for a $B \bar{B}$ event) and values that are closer to one indicates that the event approximates a jet topology (typical for a $q \bar{q}$ event).

\subsubsection{Thrust Axis Opening Angle}

Another cut parameter, $\cos \theta_{t}$, is the opening angle between the thrust axis, $\vec{T}$, and the B candidate's reconstructed momentum vector, $\vec{p}$ : 


$$
\cos \theta_{T}=\frac{\vec{p} \cdot \vec{T}}{|\vec{p}||\vec{T}|}
$$

Where the thrust axis is the direction which maximizes the thrust defined by:

$$
T=\frac{\sum_{i}\left|\widehat{T} \cdot \overrightarrow{p_{i}}\right|}{\sum_{i}\left|\overrightarrow{p_{i}}\right|}
$$

For background $q \bar{q}$ continuum events, this angle is quite small. For signal MC, there is no dependence on this variable and it is uniformly distributed.

\subsubsection{Dalitz Weight}

A $D^{0} \rightarrow K^{-} \pi^{+} \pi^{0}$ candidate must also have a minimal Dalitz weight. The Dalitz weight uses the momenta of the three daughter particles to calculate the relative decay density [61]. Figure 4.2 shows a sample Dalitz weight distribution [63].

Experiment E-691 [73] parameterized the resonant sub-structure for $D^{0} \rightarrow K^{-} \pi \pi^{0}$. The decay obeys:

$$
d \Gamma=\frac{1}{(2 \pi)^{3}} \frac{1}{32 M^{3}}|M|^{2} d m_{23}^{2} d m_{13}^{2}
$$

where $d m_{23}^{2}$ represents the invariant mass squared of the $K^{-} \pi$ combination and $d m_{13}^{2}$ is the invariant mass squared for the $K^{-} \pi^{0}$ combination. 


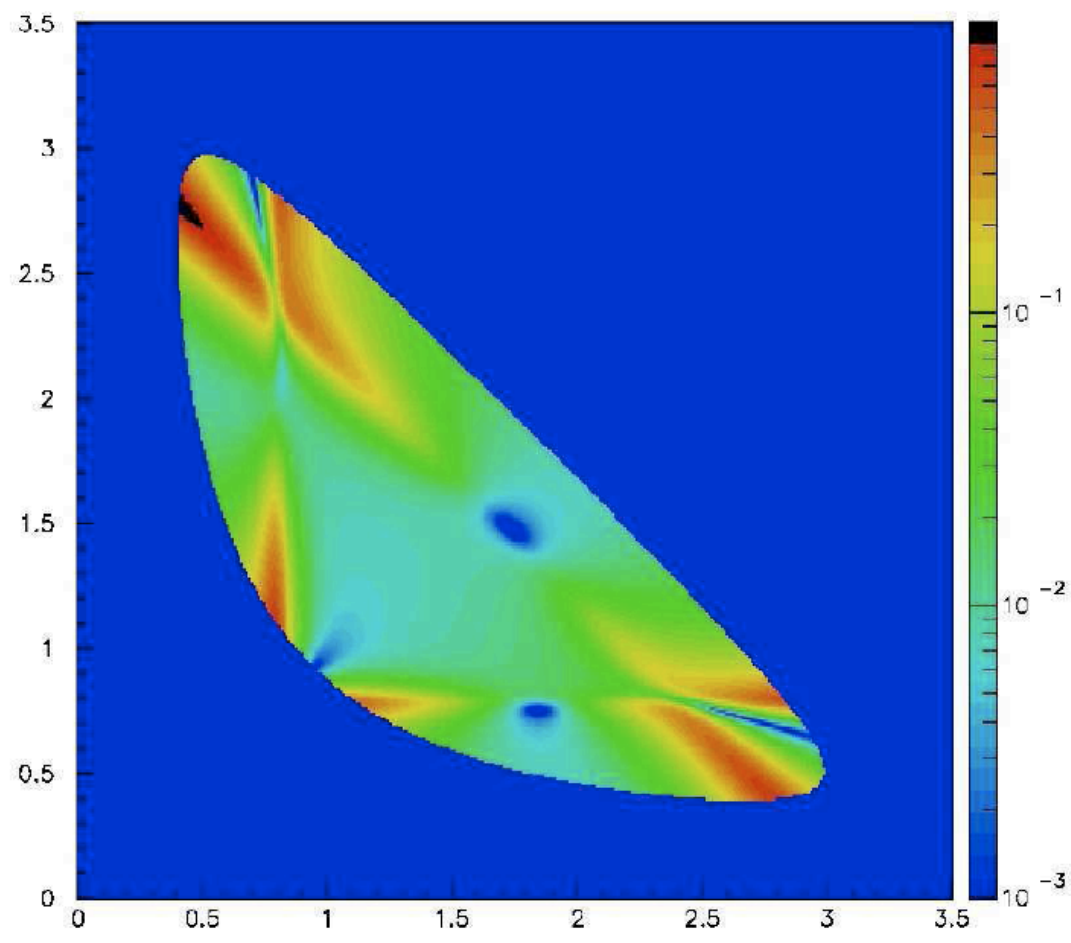

Figure 4.2: Dalitz weight distribution [63].

E-691 parameterized the matrix element according to:

$$
|M|^{2} \propto W\left(m_{13}^{2}, m_{23}^{2}\right)=\left|1+\sum_{k=1}^{n} c_{k} \exp \left(i \theta_{k}\right) F_{k}^{B W}\left(m_{13}^{2}, m_{23}^{2}\right) D_{k}^{a n g}\left(m_{13}^{2}, m_{23}^{2}\right)\right|^{2}
$$

$D_{k}^{a n g}$ represents the angular dependence of the kth resonance and $F_{k}^{B W}$ is an normalized Breit Wigner function. We use the function $W\left(m_{13}^{2}, m_{23}^{2}\right)$ to discriminate against combinatoric background to $D^{0} \rightarrow K^{-} \pi \pi^{0}$. 


\subsection{Selection Optimization}

After the events have been processed into a form that can be conveniently analyzed, the selection values are chosen using an optimization procedure. The procedure is designed to achieve the best statistical uncertainty on the branching fraction. The is accomplished by maximizing the variable $Q^{2}$ :

$$
Q^{2}=\frac{S^{2}}{S+B}
$$

where $\mathrm{S}$ is the number of signal events and $\mathrm{B}$ is the number of background events. The iterative procedure is as follows:

1. Initial selection values are applied and $Q^{2}$ is estimated.

2. The first selection variable is allowed to float until $Q^{2}$ is maximized.

3. Procedure is repeated on all other cut variables.

4. Maximization process is repeated on the new set of cut values.

5. Process continues until the value $Q^{2}$ stabilizes.

The entire optimization process is repeated using a different set of initial cut values. This is to reduce the probability of the optimized values being merely a local maximum rather than the true maximum. The above procedure produces the values listed in Table 4.5. 


\subsection{Measuring Inclusive Branching Fraction}

\subsubsection{Inclusive Measurement Calculation for $B^{-} \rightarrow D^{+} \pi^{-} \pi^{-}$}

For the measurement of $\mathcal{B}\left(B^{-} \rightarrow D^{+} \pi^{-} \pi^{-}\right)$, the expected yield of $B^{-} \rightarrow D^{+} \pi^{-} \pi^{-}\left(D^{+} \rightarrow K^{-} \pi^{+} \pi^{+}\right)$is:

$$
\begin{aligned}
& \left\langle Y_{B^{-} \rightarrow D^{+} \pi^{-} \pi^{-}\left(D^{+} \rightarrow K^{-} \pi^{+} \pi^{+}\right)}\right\rangle= \\
& N_{B^{+} B^{-}} \times\left\langle\epsilon_{B^{-} \rightarrow D^{+} \pi^{-} \pi^{-}\left(D^{+} \rightarrow K^{-} \pi^{+} \pi^{+}\right)}\right\rangle \times \mathcal{B}_{B^{-} \rightarrow D^{+} \pi^{-} \pi^{-}} \times \mathcal{B}_{D^{+} \rightarrow K^{-} \pi^{+} \pi^{+}}
\end{aligned}
$$

where:

1. $N_{B^{+} B^{-}} \equiv$ Total Number of $B^{+} B^{-}$pairs.

2. $\left\langle\epsilon_{B^{-} \rightarrow D^{+} \pi^{-} \pi^{-}\left(D^{+} \rightarrow K^{-} \pi^{+} \pi^{+}\right)}\right\rangle \equiv$ Efficiency of $B^{-} \rightarrow D^{+} \pi^{-} \pi^{-}\left(D^{+} \rightarrow\right.$ $K^{-} \pi^{+} \pi^{+}$) averaged over non-resonant and resonant contributions.

3. $\mathcal{B}_{B^{-} \rightarrow D^{+} \pi^{-} \pi^{-}} \equiv$ branching fraction of $B^{-} \rightarrow D^{+} \pi^{-} \pi^{-}$

4. $\mathcal{B}_{D^{+} \rightarrow K^{-} \pi^{+} \pi^{+}} \equiv$ branching fraction of $D^{+} \rightarrow K^{-} \pi^{+} \pi^{+}$.

Rearranging these values shows how one can measure the inclusive branching fraction of $B^{-} \rightarrow D^{+} \pi^{-} \pi^{-}\left(D^{+} \rightarrow K^{-} \pi^{+} \pi^{+}\right)$. 
$\mathcal{B}_{B^{-} \rightarrow D^{+} \pi^{-} \pi^{-}\left(D^{+} \rightarrow K^{-} \pi^{+} \pi^{+}\right)}=\frac{\left.Y_{B^{-} \rightarrow D^{+} \pi^{-} \pi^{-}\left(D^{+} \rightarrow K^{-} \pi^{+} \pi^{+}\right.}\right)}{\epsilon_{B^{-} \rightarrow D^{+} \pi^{-} \pi^{-}\left(D^{+} \rightarrow K^{-} \pi^{+} \pi^{+}\right.} \times \mathcal{B}_{D^{+} \rightarrow K^{-} \pi^{+} \pi^{+}} \times N_{B^{+} B^{-}}}$

where $Y_{B^{-} \rightarrow D^{+} \pi^{-} \pi^{-}\left(D^{+} \rightarrow K^{-} \pi^{+} \pi^{+}\right)}$is the measured yield extracted from the $\Delta E$ fit. In shorthand, one may write Equation 4.25 as:

$$
\mathcal{B}_{B^{-} \rightarrow D^{+} \pi^{-} \pi^{-}}=\frac{Y}{\epsilon \mathcal{B} N_{B^{+} B^{-}}}
$$

\subsubsection{Inclusive Measurement Calculation for $B^{-} \rightarrow D^{*+} \pi^{-} \pi^{-}$}

For the measurement of $\mathcal{B}\left(B^{-} \rightarrow D^{*+} \pi^{-} \pi^{-}\right)$, the equation used is:

$$
\mathcal{B}_{B^{-} \rightarrow D^{*+} \pi^{-} \pi^{-}}=\frac{\sum_{i} W_{i} \mathcal{B}_{i}}{\sum_{i} W_{i}}
$$

Where $W_{i}$ are the statistical weights:

$$
W_{i}=\frac{1}{\left[\frac{\delta Y_{i} \mathcal{B}_{i}}{Y_{i}}\right]^{2}}
$$

where $\delta Y$ is the statistical uncertainty on the yield and $B_{i}$ is the branching fraction corresponding to each $D^{0}$ sub-decay mode, i: 


$$
\mathcal{B}_{i}=\frac{Y_{i}}{\epsilon_{i} \mathcal{B}_{D_{i}} N_{B^{+} B^{-}}} .
$$

The efficiency for $B^{-} \rightarrow D^{*+} \pi^{-} \pi^{-}$is obtained from:

$$
\epsilon_{i}=\frac{\sum_{\alpha} \frac{V_{\alpha} Y_{i \alpha}^{M C}}{N_{i \alpha}}}{\sum_{\gamma} V_{\gamma}}
$$

with

1. $Y_{i \alpha}^{M C}$ being the $\mathrm{MC}$ yield $B^{+} \rightarrow D_{J \alpha}^{0} \pi^{+}\left(D_{J \alpha}^{0} \rightarrow D^{*+} \pi^{-}\right)$

2. $N_{i \alpha}$ being the number of $\mathrm{MC}$ events for $B^{+} \rightarrow D_{J \alpha}^{0} \pi^{+}\left(D_{J \alpha}^{0} \rightarrow D^{*+} \pi^{-}\right)$

( $\alpha \equiv$ the specific $D_{J}^{0}$ and $i \equiv$ the specific $D^{0}$ decay mode) And:

$$
V_{\alpha}=\left\{B\left(B^{-} \rightarrow D_{J \alpha}^{0} \pi^{-}\right) \times B\left(D_{J \alpha}^{0} \rightarrow D^{*+} \pi^{-}\right)\right\}
$$

as measured by BELLE, the B-factory experiment currently running in Japan.

\subsection{Uncertainties}

For the results, we report three numbers. The first is the central value. It is the 'measured' value in this analysis. There are also two 'uncertainties'. The first is the 'statistical' uncertainty which depends on the size of the 
available data set. The second is the systematic uncertainty. The systematic uncertainties are due to the detector effects as well as the analysis technique.

\subsubsection{Statistical Uncertainty}

The statistical uncertainty for $\mathcal{B}\left(B^{-} \rightarrow D^{*+} \pi^{-} \pi^{-}\right)$is given by:

$$
\delta\left[\mathcal{B}\left(B^{-} \rightarrow D^{*+} \pi^{-} \pi^{-}\right)\right]=\frac{1}{\sqrt{\left(\sum_{k} W_{k}\right)}}
$$

The statistical uncertainty on $\mathcal{B}\left(B^{-} \rightarrow D^{*+} \pi^{-} \pi^{-}\right)$is:

$$
\delta\left[\mathcal{B}\left(B^{-} \rightarrow D^{*+} \pi^{-} \pi^{-}\right)\right]=\frac{\delta Y}{Y} .
$$

\subsubsection{Systematic Uncertainties}

In this analysis, the systematic uncertainties considered are:

1. B-counting.

The number of $B^{+} B^{-}$pairs in the data sample is not perfectly known. The systematic uncertainty due to the B-counting is estimated based a on previous study [46]. 
2. Branching fractions of the $D^{*+}, D^{+}$, and $D^{0}$.

The intermediate branching fractions.

3. Signal MC.

Uncertainty of the fitted values used for the efficiencies.

4. Efficiency differences among the resonant and non-resonant decays.

The efficiencies of the signal MC can vary.

5. Multiple candidates.

Each event may produce more than one B-candidate. A procedure picks the 'best' one.

6. Tracking Efficiency for charged pions and kaons.

$B A B A R$ is not $100 \%$ efficient in identifying these particles.

7. Particle Identification.

8. $\pi^{0}$ mass smearing.

Their respective systematic errors were measured and are shown on Table 4.12 . 


\subsubsection{B-Counting}

$B A B A R$ is a $\mathrm{B}$ meson factory. Its main purpose is to reconstruct $\mathrm{B}$ events and record them. But the detector does not record and reconstruct all of them and some reconstructed B's may be 'false positives'.

A working group was assigned the task of determining this uncertainty. This group studied the number of hadronic events in a given sample and compared it to the number of muon pairs in an on resonance data sample and an off resonance data sample. Through the use of these values, the number of $\Upsilon(4 S)$ particles may be counted. The group took the following into account for the uncertainty.

1. Efficiency for GoodTracksLoose and other hadronic selections - Contribution of the uncertainty of the cut variables that select hadronic events.

2. Vertexing - Contributed by the changing of the vertexing code over time.

3. DCH Voltage - Uncertainty contributed by the variance in the voltage of the drift chamber.

4. Damaged DCH Events - Uncertainty contributed by a fraction of events with a sufficient number of hits to overflow the $\mathrm{DCH}$ readout module.

5. BGF MultiHadron - Uncertainty in the efficiency of BGFMultiHadron 
(Number of tracks for the event is greater than 2 and $R_{2}<0.5$ ).

The uncertainty due the B counting is $1.1 \%$ and is dominated by systematic uncertainties.

\subsection{4 $D^{*+}, D^{0}, D^{+}$Branching Fraction Uncertainties}

The Branching Fractions for $D^{*}, D^{0}$, and $D^{+}$and their respective uncertainties come from PDG 2006 [80]. These three particles have been studied by many other collaborations. Their respective branching fractions and uncertainties are shown in Table 4.6.

\begin{tabular}{|c|c|c|}
\hline Mode & $\mathcal{B}(\%)$ & $\delta \mathcal{B}(\%)$ \\
\hline$D^{0} \rightarrow K^{-} \pi^{+}$ & 3.80 & 0.07 \\
$D^{0} \rightarrow K^{-} \pi^{+} \pi^{0}$ & 14.1 & 0.5 \\
$D^{0} \rightarrow K^{-} \pi^{+} \pi^{-} \pi^{+}$ & 7.72 & 0.28 \\
$D^{+} \rightarrow K^{-} \pi^{+} \pi^{+}$ & 9.51 & 0.34 \\
$D^{*} \rightarrow D^{0} \pi$ & 67.7 & 0.5 \\
\hline
\end{tabular}

Table 4.6: Branching fraction and uncertainties for $D^{0}, D^{+}$, and $D^{*+}$.

The systematic errors are measured by using:

$$
\text { Precision }=\frac{\delta \mathcal{B}}{\mathcal{B}}
$$




\subsubsection{Uncertainties on Efficiencies}

Each of the submodes for Table 4.7 has an efficiency that is determined from Signal MC data. The systematic error contribution is the ratio of the uncertainty of the MC yield with the MC yield:

\begin{tabular}{|l|l|}
\hline$B^{-} \rightarrow D^{*+} \pi^{-} \pi^{-}$ & $B^{-} \rightarrow D^{+} \pi^{-} \pi^{-}$ \\
\hline$D^{*+} \rightarrow D^{0} \pi^{+}$ & $D^{+} \rightarrow K^{-} \pi^{+} \pi^{+}$ \\
$D^{0} \rightarrow K^{-} \pi^{+}$ & \\
$D^{0} \rightarrow K^{-} \pi^{+} \pi^{0}, \pi^{0} \rightarrow \gamma \gamma$ & \\
$D^{0} \rightarrow K^{-} \pi^{+} \pi^{-} \pi^{+}$ & \\
\hline
\end{tabular}

Table 4.7: Submodes used for efficiency measurements.

$$
\text { Precision }=\frac{\delta Y}{Y}
$$

The component parts are then weighted based upon their respective branching fractions in a recent Belle publication. 


\begin{tabular}{|c|c|}
\hline Mode & Fraction $\%$ \\
\hline$D^{0} \rightarrow K^{-} \pi^{+}$ & 0.27 \\
$D^{0} \rightarrow K^{-} \pi^{+} \pi^{0}$ & 0.28 \\
$D^{0} \rightarrow K^{-} \pi^{+} \pi^{-} \pi^{+}$ & 0.49 \\
$D^{+} \rightarrow K^{-} \pi^{+} \pi^{+}$ & 0.21 \\
\hline
\end{tabular}

Table 4.8: MC Statistics Systematic Errors.

\subsubsection{Efficiency Differences}

The inclusive branching fractions is a combination of their component modes. As in the case of $B^{-} \rightarrow D^{*+} \pi^{-} \pi^{-} ; D^{*+} \rightarrow D^{0} \pi^{+}, D^{0} \rightarrow K^{-} \pi^{+}$, $D^{0} \rightarrow K^{-} \pi^{+} \pi^{0}$ and $D^{0} \rightarrow K^{-} \pi^{+} \pi^{-} \pi^{+}$each have their own efficiency and uncertainty. Difference between efficiencies calculated from signal MC with different $D_{J}^{0}$ states. Therefore, an additional uncertainty must be applied that is based on the differences of the efficiencies. For each submode, the signal MC component with the highest and lowest uncertainty is determined. They are then averaged and given an uncertainty based on half the difference between these two efficiencies. The systematic error is this uncertainty divided by the average.

$$
\text { Systematic }=\frac{0.5\left(\epsilon_{\text {high }}-\epsilon_{\text {low }}\right)}{\epsilon_{\text {Average }}}
$$




\begin{tabular}{|c|c|c|c|}
\hline$D^{0} \rightarrow K^{-} \pi^{+}$ & $D^{0} \rightarrow K^{-} \pi^{+} \pi^{0}$ & $D^{0} \rightarrow K^{-} \pi^{+} \pi^{-} \pi^{+}$ & $D^{+} \rightarrow K^{-} \pi^{+} \pi^{+}$ \\
\hline $3.5 \%$ & $2.1 \%$ & $3.1 \%$ & $3.0 \%$ \\
\hline
\end{tabular}

Table 4.9: Efficiency differences.

\subsubsection{Multiple Candidates}

In this analysis, events may exist in which there are more than one reconstructed B-candidate. When this occurs, the one with the best $\chi^{2}$ from the vertex fit is chosen. An alternative technique would be picking the best B-candidate by the mass of the reconstructed $D$. Each technique is used and the sub-mode's efficiencies are estimated. The systematic uncertainty due to the best candidate selection was set to be the difference between these two values. This shows that the multiple candidate systematic uncertainty is not a significant contributor.

\begin{tabular}{|c|c|c|c|}
\hline$D^{0} \rightarrow K^{-} \pi^{+}$ & $D^{0} \rightarrow K^{-} \pi^{+} \pi^{0}$ & $D^{0} \rightarrow K^{-} \pi^{+} \pi^{-} \pi^{+}$ & $D^{+} \rightarrow K^{-} \pi^{+} \pi^{+}$ \\
\hline $0.040 \%$ & $0.46 \%$ & $0.24 \%$ & $0.082 \%$ \\
\hline
\end{tabular}

Table 4.10: Systematic uncertainty of the multiple candidates.

\subsubsection{Tracking Efficiencies}

The tracking efficiency group assigns a systematic uncertainty to the reconstruction efficiency for each of the tracks used in the reconstruction of the $\mathrm{B}$ candidate. Each is based on the variety of the track being used. Since 
the tracks used in this analysis are GoodTracksLoose, a 1.0\% uncertainty is assigned per track. So a 5 track event is measured to have a $5 \%$ systematic uncertainty. Table 4.11 provides the resulting sums.

\begin{tabular}{|c|c|}
\hline Mode, $B^{-} \rightarrow D^{*+} \pi^{-} \pi^{-}$or $B^{-} \rightarrow D^{+} \pi^{-} \pi^{-}$with & Relative Systematic Error (\%) \\
\hline$D^{0} \rightarrow K^{-} \pi^{+}$ & 5.0 \\
$D^{0} \rightarrow K^{-} \pi^{+} \pi^{0}$ & 5.0 \\
$D^{0} \rightarrow K^{-} \pi^{+} \pi^{-} \pi^{+}$ & 7.0 \\
$D^{+} \rightarrow K^{-} \pi^{+} \pi^{+}$ & 5.0 \\
\hline
\end{tabular}

Table 4.11: Systematic Uncertainty due to Tracking.

\subsubsection{Particle Identification}

The proper particle identification (PID) of kaons used in $D^{0}$ and $D^{+}$ reconstruction has an uncertainty. The kaon identification, like the pions, have a particle identification efficiency and uncertainty. The detector is unable to positively identify all kaons. And it is understood that there is an uncertainty on how effective the PID system is in identifying kaons. From the PID 'rules of thumb' documentation [65], the uncertainty of $1.2 \%$ for the identification efficiency for kaons is established. The usage of this number is permitted through the fact that the kaons used in this analysis, as in the 'rules of thumb' have a similar momentum range and KLHTight conditions. 


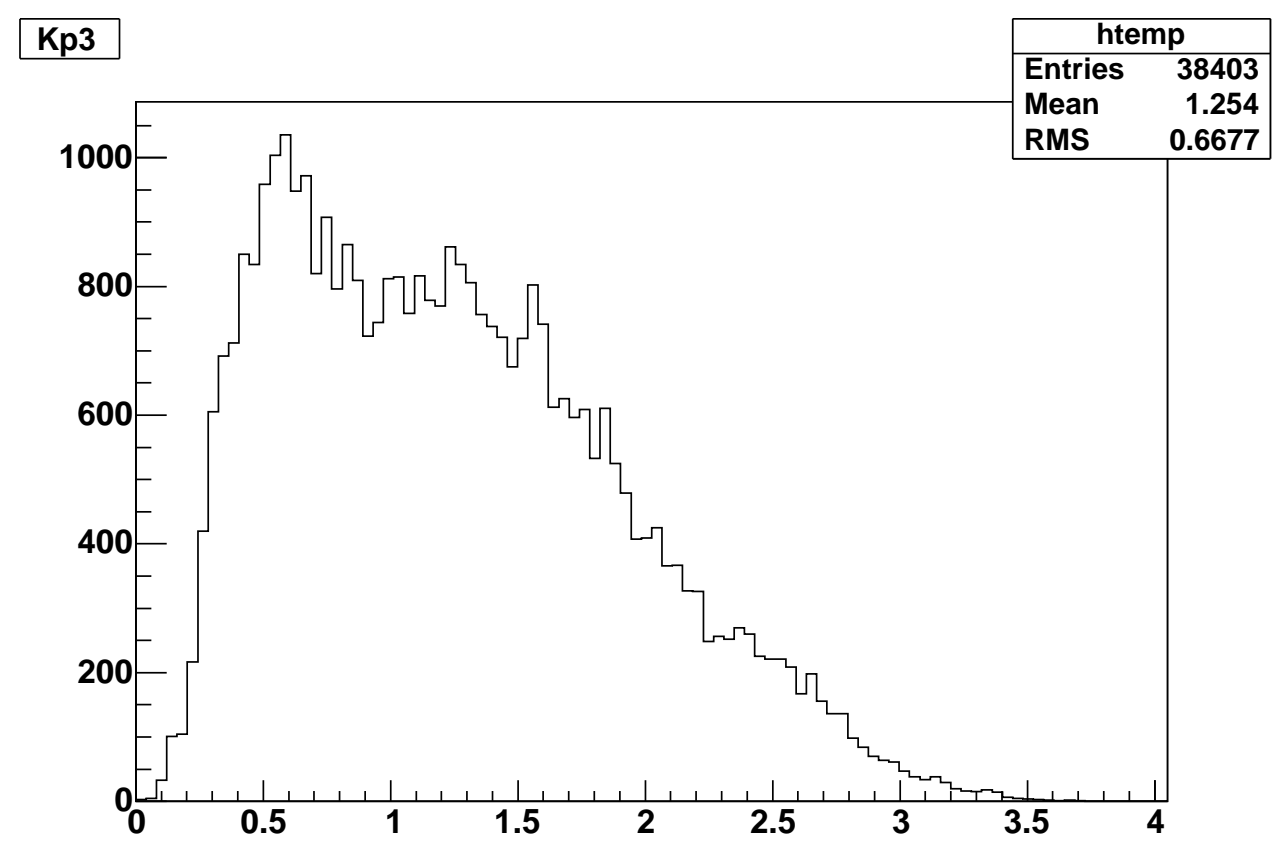

Figure 4.3: Kaon momentum spectrum of $B^{-} \rightarrow D^{*+} \pi^{-} \pi^{-}\left(D^{0} \rightarrow K^{-} \pi^{+}\right)$.

\subsubsection{0 $\pi^{0}$ Mass Resolution}

The systematic errors concerning the $\pi^{0}$ only affects the mode $D^{0} \rightarrow$ $K^{-} \pi^{+} \pi^{0}$, but this error will later propagate into the $\mathcal{B}\left(B^{-} \rightarrow D^{*+} \pi^{-} \pi^{-}\right)$ measurements. This error, as stated by the neutral AWG recipe web page [66], places a $3 \%$ systematic uncertainty, plus an additional factor is based on a recipe that uses the lab frame momentum spectrum of the $\pi^{0}$. 


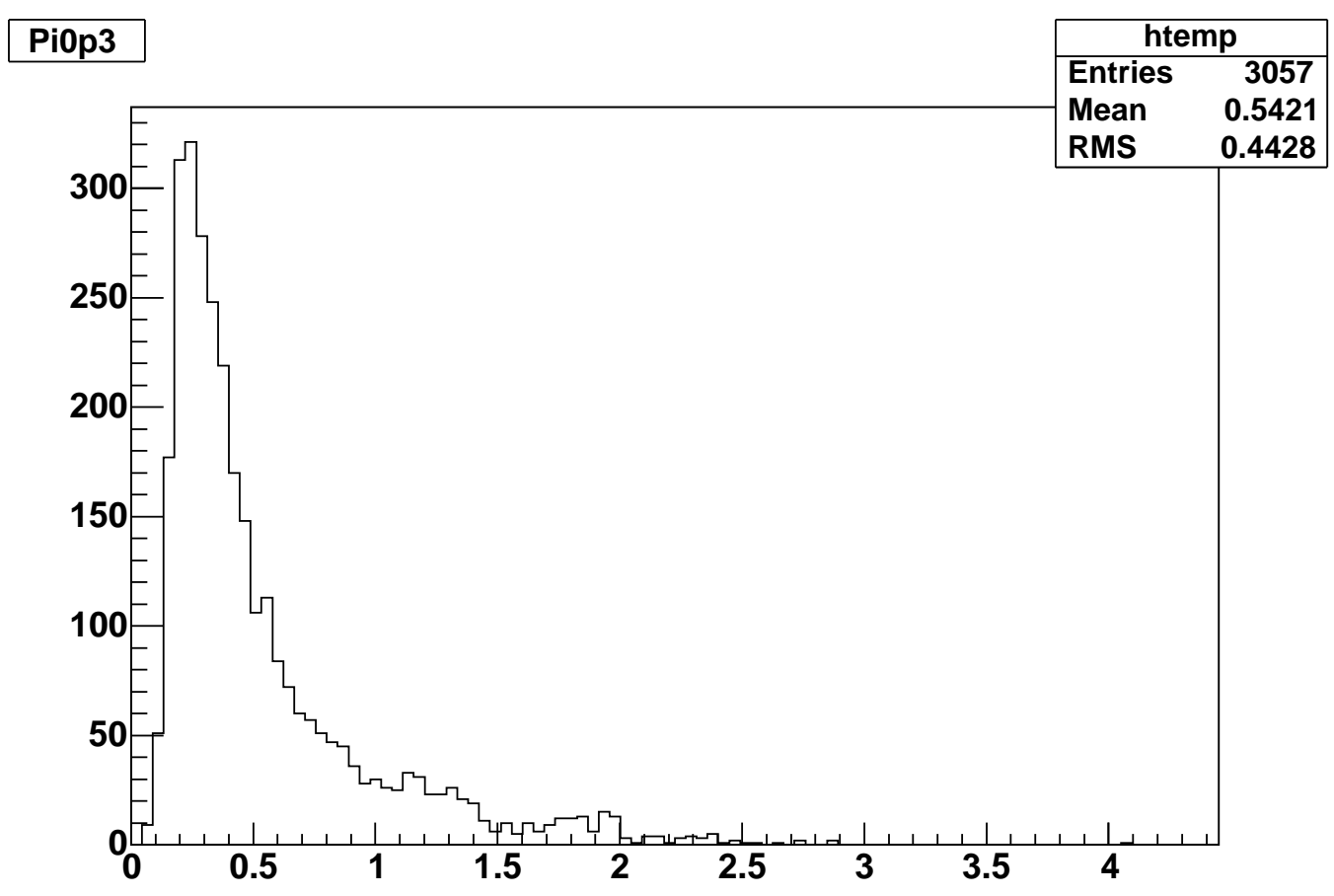

Figure 4.4: The $\pi^{0}$ momentum spectrum of the data for the decay of $B^{-} \rightarrow$ $D^{*+} \pi^{-} \pi^{-}\left(D^{0} \rightarrow K^{-} \pi^{+} \pi^{0}\right)$.

\begin{tabular}{|c|c|c|c|c|}
\hline Source & $D^{0} \rightarrow K^{-} \pi^{+}$ & $D^{0} \rightarrow K^{-} \pi^{+} \pi^{0}$ & $D^{0} \rightarrow K^{-} \pi^{+} \pi^{-} \pi^{+}$ & $D^{+} \rightarrow K^{-} \pi^{+} \pi^{+}$ \\
\hline B-counting & $1.10 \%$ & $1.10 \%$ & $1.10 \%$ & $1.10 \%$ \\
\hline Tracking efficiency & $5.0 \%$ & $5.0 \%$ & $7.0 \%$ & $5.0 \%$ \\
\hline PID & $1.20 \%$ & $1.20 \%$ & $1.20 \%$ & $1.20 \%$ \\
\hline $\mathcal{B}\left(D^{*+}\right)$ & $0.70 \%$ & $0.70 \%$ & $0.70 \%$ & - \\
\hline Efficiency differences & $<1.0 \%$ & $<1.0 \%$ & $<1.0 \%$ & $<1.0 \%$ \\
\hline Multiple candidates & $<1.0 \%$ & $<1.0 \%$ & $<1.0 \%$ & $<1.0 \%$ \\
\hline$\pi^{0}$ smearing & - & $3.0 \%$ & - & - \\
\hline $\mathcal{B}\left(D^{0}\right)$ & $1.8 \%$ & $3.5 \%$ & $3.60 \%$ & - \\
\hline $\mathcal{B}\left(D^{+}\right)$ & - & - & - & $3.6 \%$ \\
\hline MC statistics & $<1.0 \%$ & $<1.0 \%$ & $<1.0 \%$ & $<1.0 \%$ \\
\hline Sub-mode total & $6.61 \%$ & $7.34 \%$ & $8.67 \%$ & \\
\hline Total Systematic Error & $B^{-} \rightarrow D^{*+} \pi^{-} \pi^{-}$ & $7.6 \%$ & $B^{-} \rightarrow D^{+} \pi^{-} \pi^{-}$ & $7.1 \%$ \\
\hline
\end{tabular}

Table 4.12: Relative systematic uncertainties of $\mathcal{B}\left(B^{-} \rightarrow D^{*+} \pi^{-} \pi^{-}\right)$and $\mathcal{B}\left(B^{-} \rightarrow\right.$ $\left.D^{+} \pi^{-} \pi^{-}\right)$with $D^{0} \rightarrow K^{-} \pi^{+}, D^{0} \rightarrow K^{-} \pi^{+} \pi^{0}, D^{0} \rightarrow K^{-} \pi^{+} \pi^{-} \pi^{+}$, and $D^{+} \rightarrow$ $K^{-} \pi^{+} \pi^{+}$. 


\section{Chapter 5}

\section{Results, Summary and}

\section{Conclusion}

\section{$5.1 \quad$ Results}

Using the data collected by the BABAR detector for Runs 1-5 with total integrated luminosity of $343.38 \mathrm{fb}^{-1}$, which corresponds to 383.92 million $B \bar{B}$ pairs, this thesis reports the inclusive branching fraction and supporting measurements for:

1. $\mathcal{B}\left(B^{-} \rightarrow D^{+} \pi^{-} \pi^{-}\right)$

2. $\mathcal{B}\left(B^{-} \rightarrow D^{*+} \pi^{-} \pi^{-}\right)$. 


\section{$5.2 \quad D^{0}$ and $D^{+}$Reconstructions}

The first stage is the reconstruction of the $D^{0}$ and the $D^{+}$candidates. The $D^{0}$ uses the following final decay modes:

1. $D^{0} \rightarrow K^{-} \pi^{+}$

2. $D^{0} \rightarrow K^{-} \pi^{+} \pi^{0}$

3. $D^{0} \rightarrow K^{-} \pi^{+} \pi^{-} \pi^{+}$

The $D^{+}$uses the final decay mode:

$D^{+} \rightarrow K^{-} \pi^{+} \pi^{+}$

The $D^{0}$ mass distributions, $m\left(D^{0}\right)$, are shown in Figures 5.1 - 5.3. The fitting is done using a Gaussian for the signal and a zeroth or a first order polynomial for the background. The backgrounds consists of continuum, combinatoric and peaking background. The continuum background is minimized by using the ratio of the Fox-Wolfram second moment to the zeroth $\left(R_{2}\right)$. The combinatoric background is coming from the random combination of the kaons and the pions. The $m_{e s}$ and the various mass cuts are effective at reducing the combinatoric background. The peaking background is discussed in detail in 5.4.2.

The fitting results for both real data and generic MC samples are shown in Table 5.1. The $D^{+}$mass distribution, $m\left(D^{+}\right)$, is shown in Figure 5.4. The 
value of the mass of the $D^{0}$ is set to be $1.8645 \mathrm{GeV}$. The $D^{0}$ mass must be within a specified range of the peak of the spectrum as seen in Equations 4.8 -4.10 .

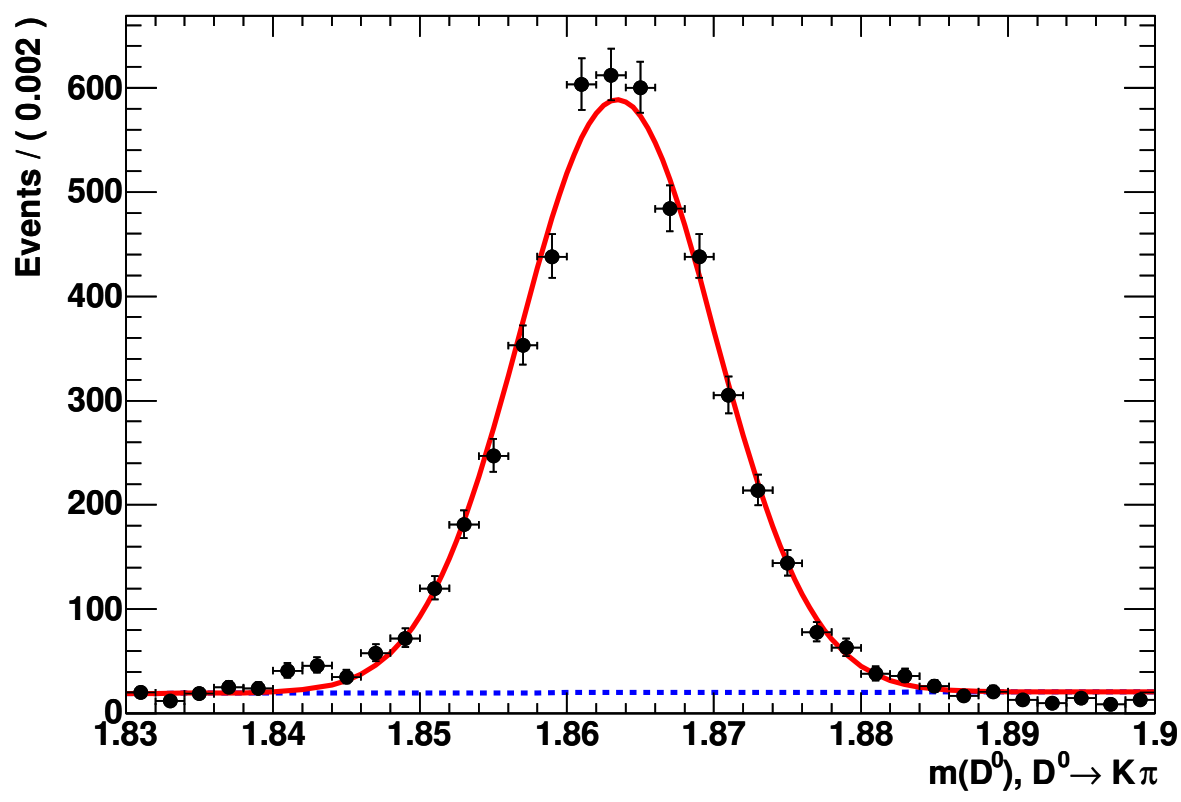

Figure 5.1: The $D^{0}$ mass distribution of $D^{0} \rightarrow K^{-} \pi^{+}$.

The signature of $D^{+} \rightarrow K^{-} \pi^{+} \pi^{+}$decays is used to reconstruct $B^{-} \rightarrow$ $D^{+} \pi^{-} \pi^{-}$decays. Figure 5.4 shows the mass spectra of $m\left(D^{+}\right)$.

\begin{tabular}{|l|c|c|c|c|}
\hline \multirow{2}{*}{ Mode } & \multicolumn{2}{|c|}{ Data } & \multicolumn{2}{c|}{ Generic Monte Carlo } \\
\cline { 2 - 5 } & Mass $\left(\mathrm{MeV} / c^{2}\right)$ & $\sigma\left(\mathrm{MeV} / c^{2}\right)$ & Mass $\left(\mathrm{MeV} / c^{2}\right)$ & $\sigma\left(\mathrm{MeV} / c^{2}\right)$ \\
\hline$D^{0} \rightarrow K^{-} \pi^{+}$ & $1863.4 \pm 0.1$ & $6.6 \pm 0.0$ & $1864.5 \pm 0.0$ & $6.5 \pm 0.0$ \\
$D^{0} \rightarrow K^{-} \pi^{+} \pi^{0}$ & $1863.1 \pm 0.2$ & $12.3 \pm 0.3$ & $1862.0 \pm 0.1$ & $11.2 \pm 0.1$ \\
$D^{0} \rightarrow K^{-} \pi^{+} \pi^{-} \pi^{+}$ & $1863.3 \pm 0.1$ & $5.0 \pm 0.1$ & $1864.5 \pm 0.0$ & $4.9 \pm 0.0$ \\
\hline
\end{tabular}

Table 5.1: The $D^{0}$ fit results for data and generic Monte Carlo. The generated value of the mass for the generic and signal MC is set to be $1864.5 \mathrm{MeV} / c^{2}$. 


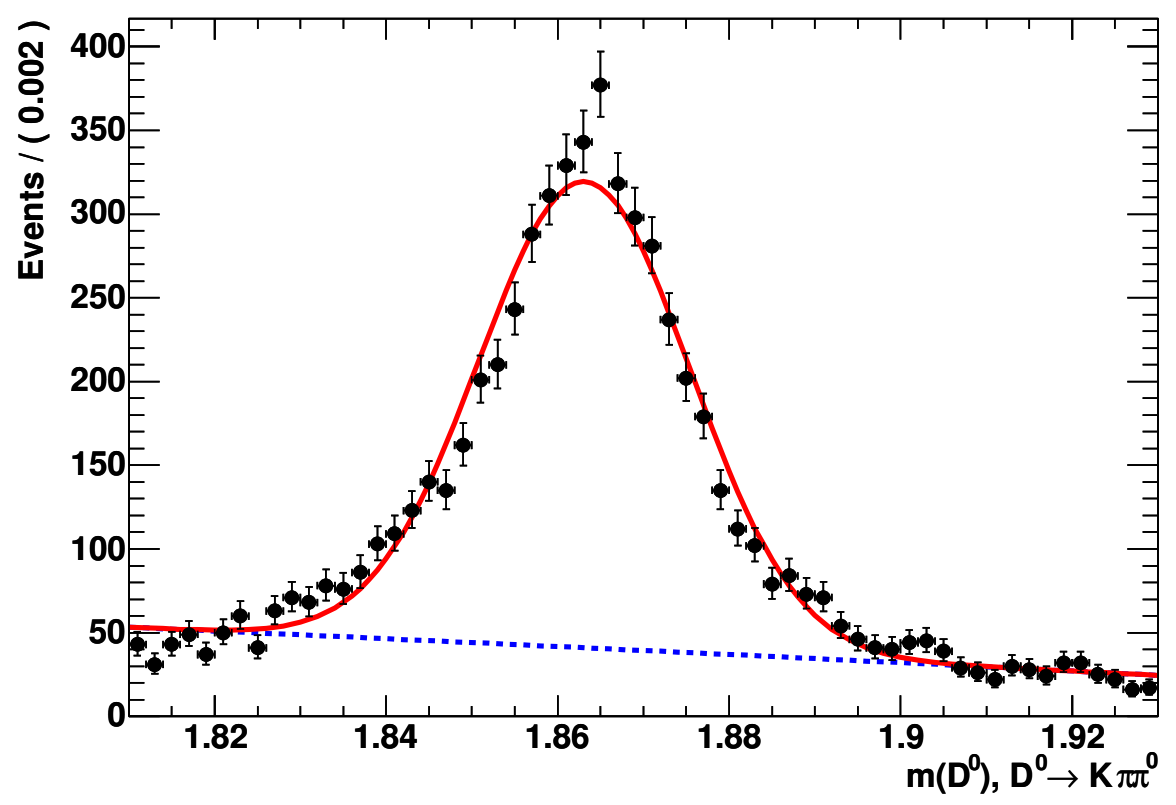

Figure 5.2: The $D^{0}$ mass distribution of $D^{0} \rightarrow K^{-} \pi^{+} \pi^{0}$.

\section{$5.3 \Delta\left(m\left(D^{*+}\right)-m\left(D^{0}\right)\right)$ Mass Difference}

The $D^{*+}$ candidate is reconstructed by combining a $D^{0}$ candidate and a $\pi^{+}$candidate. The mass difference, $\Delta\left(m\left(D^{*+}\right)-m\left(D^{0}\right)\right)$, of these candidates is shown in Figures 5.5 through 5.7, where the dotted line is the data, the solid line is the signal, and the dashed line is the combinatoric background. Candidates are fitted using a double Gaussian for the signal and a threshold background function. Final candidates must have a mass difference within a specific range of the peak as given in Equations 4.11 - 4.13. Fitted values for real data and generic MC are shown in Table 5.2. 


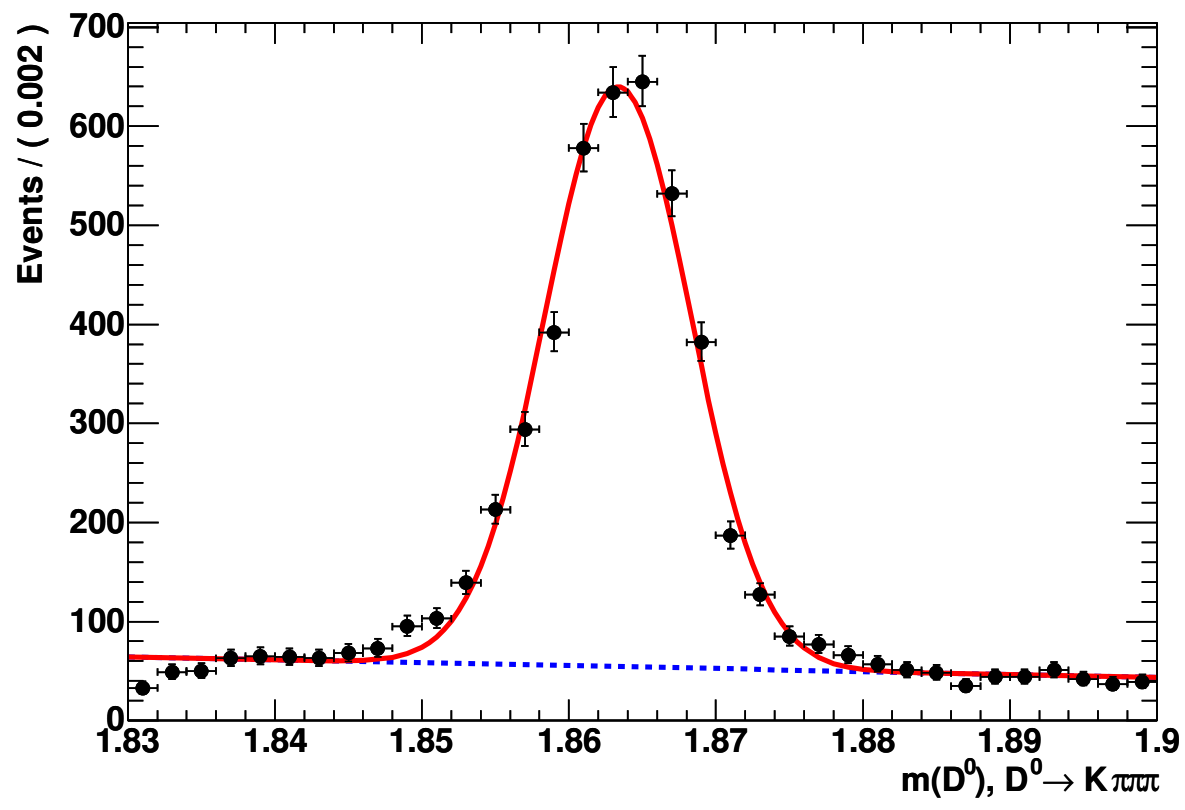

Figure 5.3: The $D^{0}$ mass distribution of $D^{0} \rightarrow K^{-} \pi^{+} \pi^{-} \pi^{+}$.

\begin{tabular}{|l|c|c|c|c|}
\hline & \multicolumn{2}{|c|}{ Data } & \multicolumn{2}{c|}{ Monte Carlo } \\
\hline$D^{*}$ decay Mode & $\Delta M\left(\mathrm{MeV} / c^{2}\right)$ & $\sigma\left(\mathrm{MeV} / c^{2}\right)$ & $\Delta M\left(\mathrm{MeV} / c^{2}\right)$ & $\sigma\left(\mathrm{MeV} / c^{2}\right)$ \\
\hline$D^{0} \rightarrow K^{-} \pi^{+}$ & $145.46 \pm 0.01$ & $0.49 \pm 0.03$ & $145.53 \pm 0.00$ & $0.65 \pm 0.01$ \\
$D^{0} \rightarrow K^{-} \pi^{+} \pi^{0}$ & $145.50 \pm 0.01$ & $0.62 \pm 0.02$ & $145.53 \pm 0.00$ & $0.64 \pm 0.01$ \\
$D^{0} \rightarrow K^{-} \pi^{+} \pi^{-} \pi^{+}$ & $145.47 \pm 0.01$ & $0.49 \pm 0.03$ & $145.52 \pm 0.00$ & $0.61 \pm 0.01$ \\
\hline
\end{tabular}

Table 5.2: $\Delta\left(m\left(D^{*+}\right)-m\left(D^{0}\right)\right)$ measurements. 


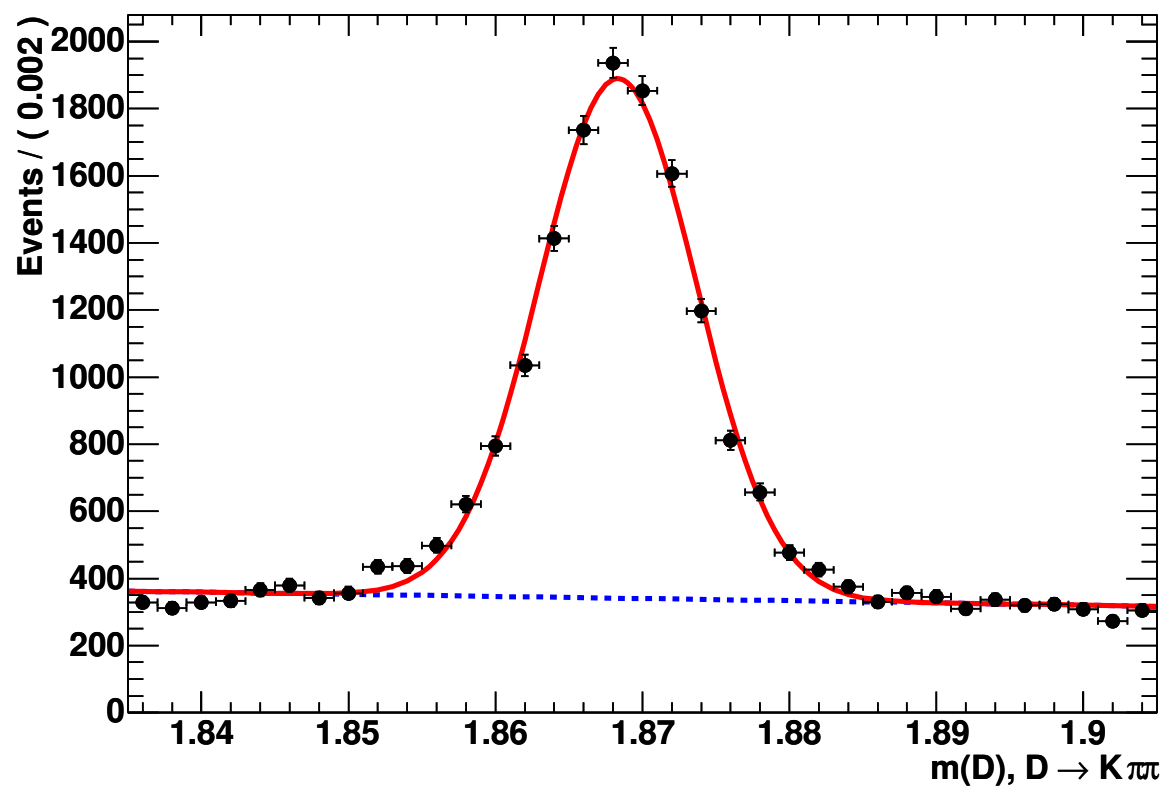

Figure 5.4: The $D^{+}$mass distribution of $D^{+} \rightarrow K^{-} \pi^{+} \pi^{+}$.

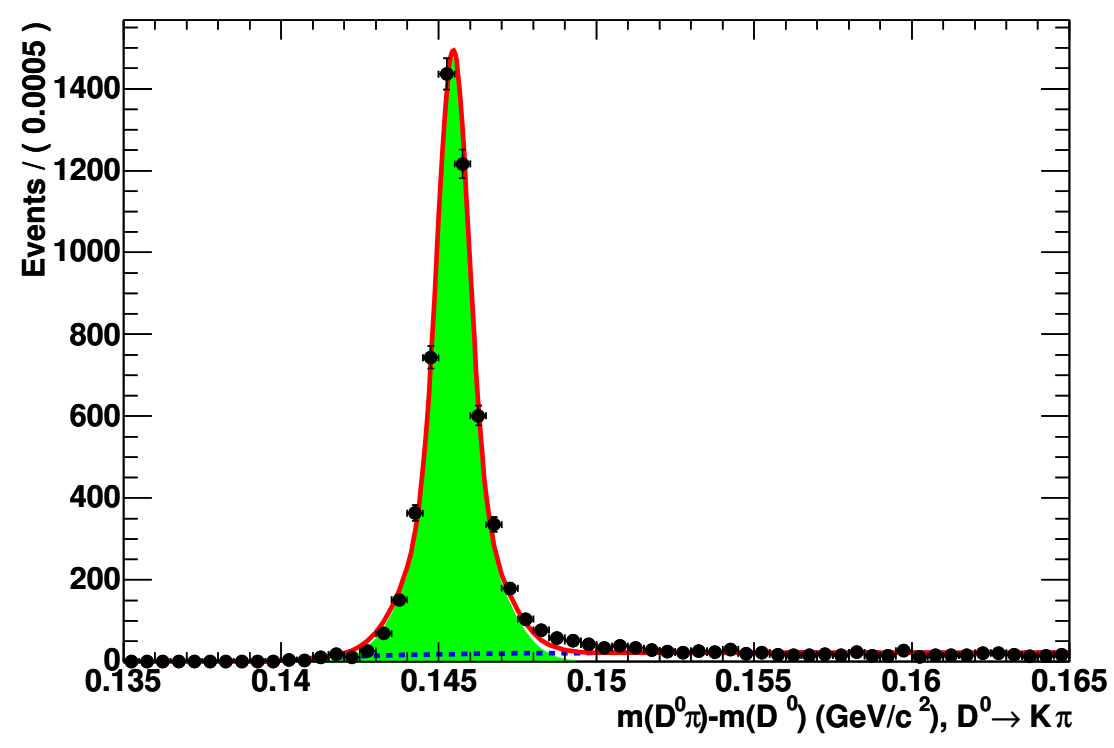

Figure 5.5: $\Delta\left(m\left(D^{*+}\right)-m\left(D^{0}\right)\right)$ mass difference with $D^{0} \rightarrow K^{-} \pi^{+}$. 


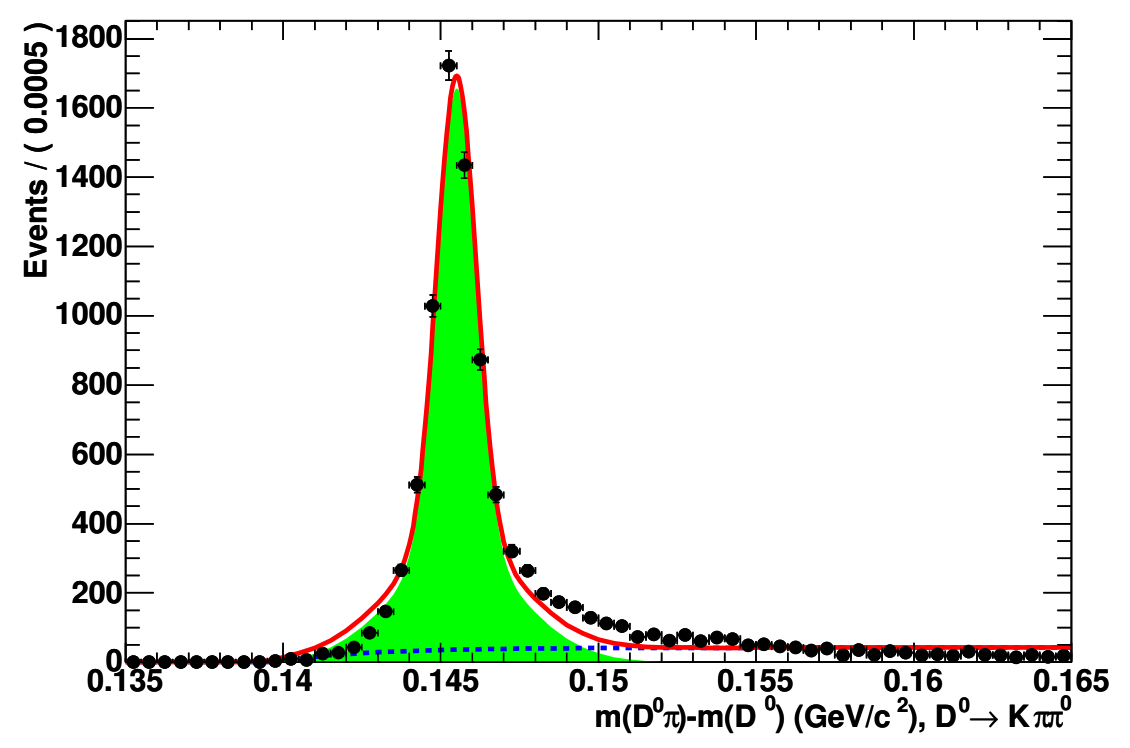

Figure 5.6: $\Delta\left(m\left(D^{*+}\right)-m\left(D^{0}\right)\right)$ mass difference with $D^{0} \rightarrow K^{-} \pi^{+} \pi^{0}$.

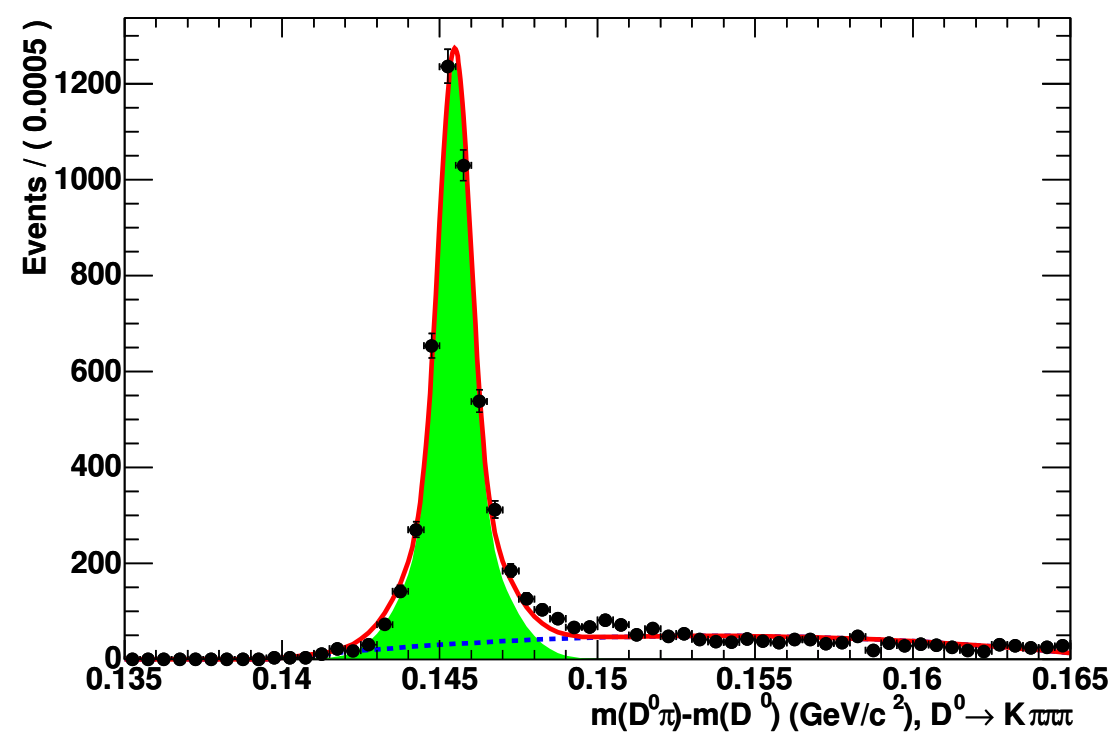

Figure 5.7: $\Delta\left(m\left(D^{*+}\right)-m\left(D^{0}\right)\right)$ mass difference with $D^{0} \rightarrow K^{-} \pi^{+} \pi^{-} \pi^{+}$. 


\subsection{The Distributions of $m_{E S}$ and $\Delta E$}

The $B^{+}$candidates are reconstructed by combining a $D^{*+}$ or $D^{+}$with two charged pions. The $m_{\mathrm{ES}}$ and $\Delta E$ distributions are measured through the following equations

$$
m_{\mathrm{ES}}=\sqrt{\left(E_{\text {beam }}^{*}\right)^{2}-\left(\sum_{i} \vec{p}_{i}^{*}\right)^{2}}
$$

and

$$
\Delta E=\sum_{i} \sqrt{m_{i}^{2}+\left(\vec{p}_{i}^{*}\right)^{2}}-E_{\text {beam }}^{*}
$$

Details of Equations 5.1 and 5.2 are discussed in Section 4.8.6. The $m_{\mathrm{ES}}$ distribution is fitted using a threshold function and a Gaussian, as shown in figures 5.9 through 5.11. The $\Delta E$ distributions are shown in figures 5.12 through 5.15 . 


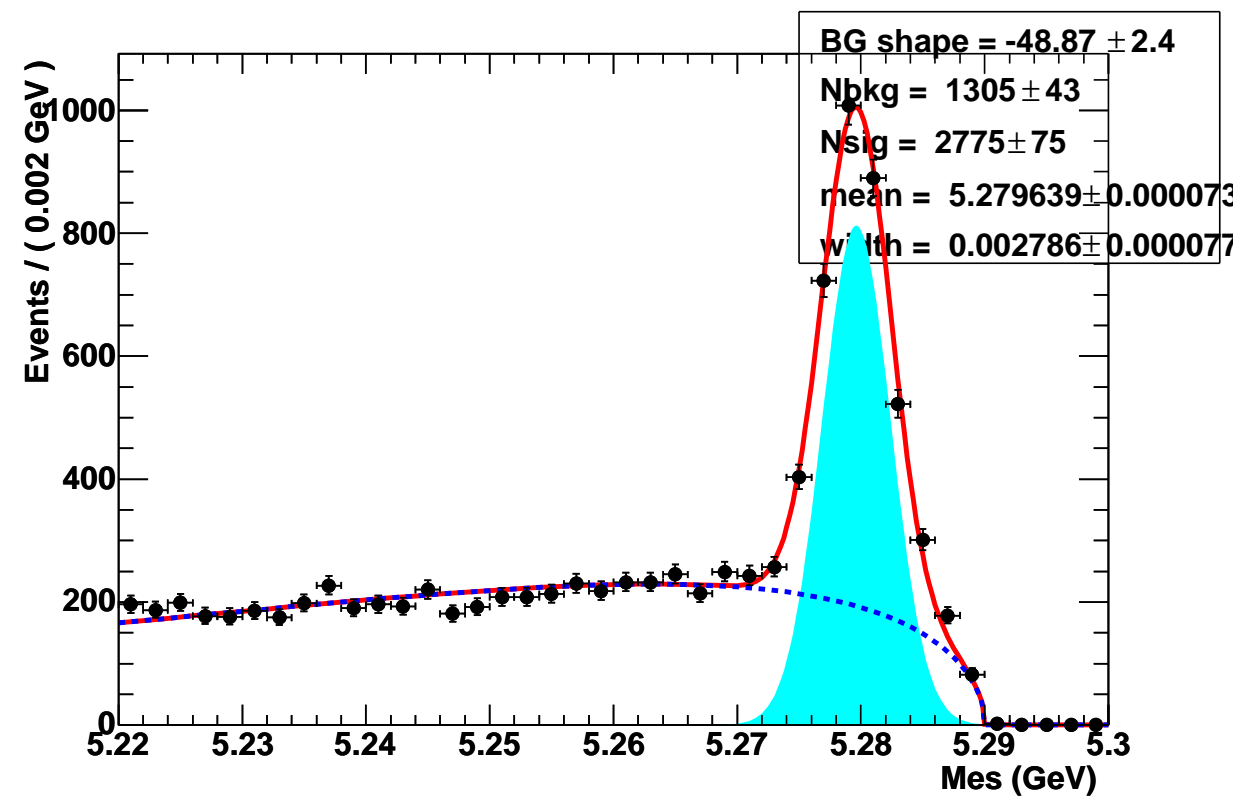

Figure 5.8: The $m_{\mathrm{ES}}$ distribution using $D^{0} \rightarrow K^{-} \pi^{+}$.

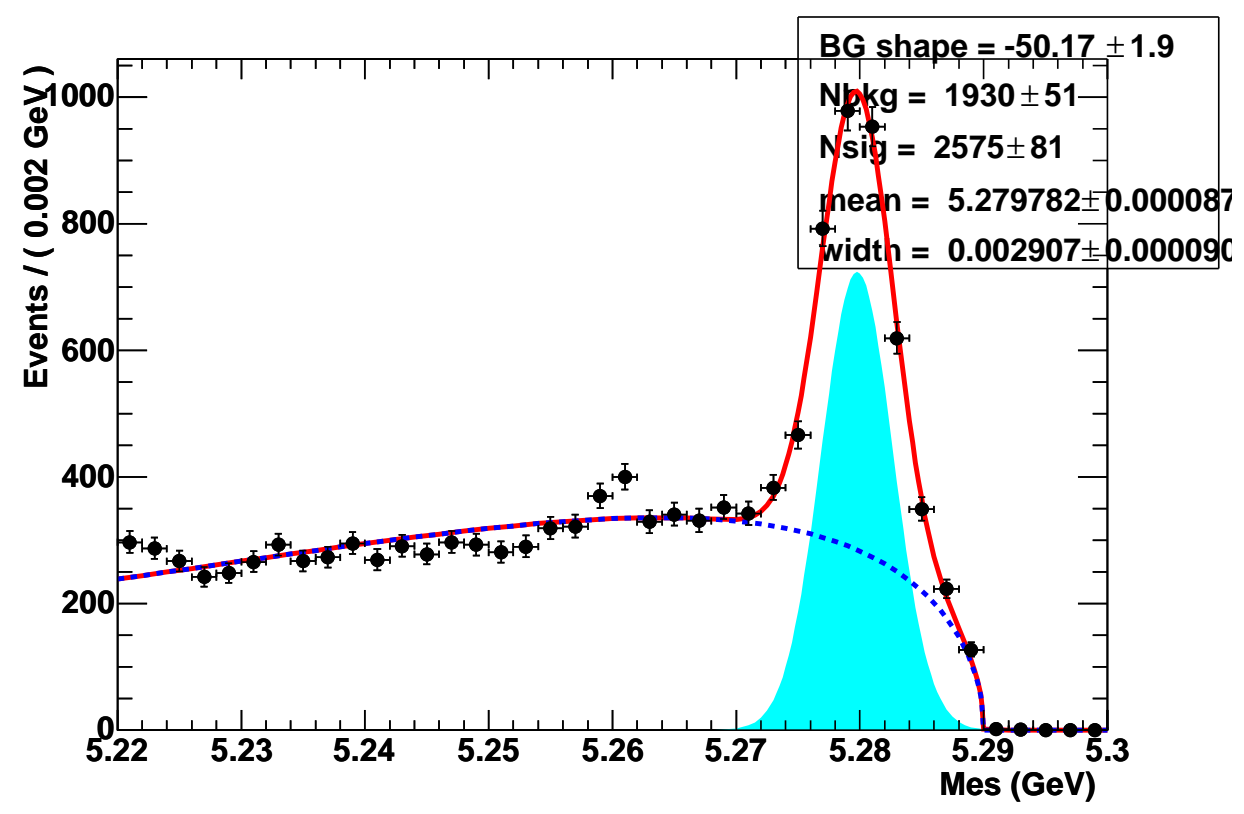

Figure 5.9: The $m_{\mathrm{ES}}$ distribution using $D^{0} \rightarrow K^{-} \pi^{+} \pi^{0}$. 


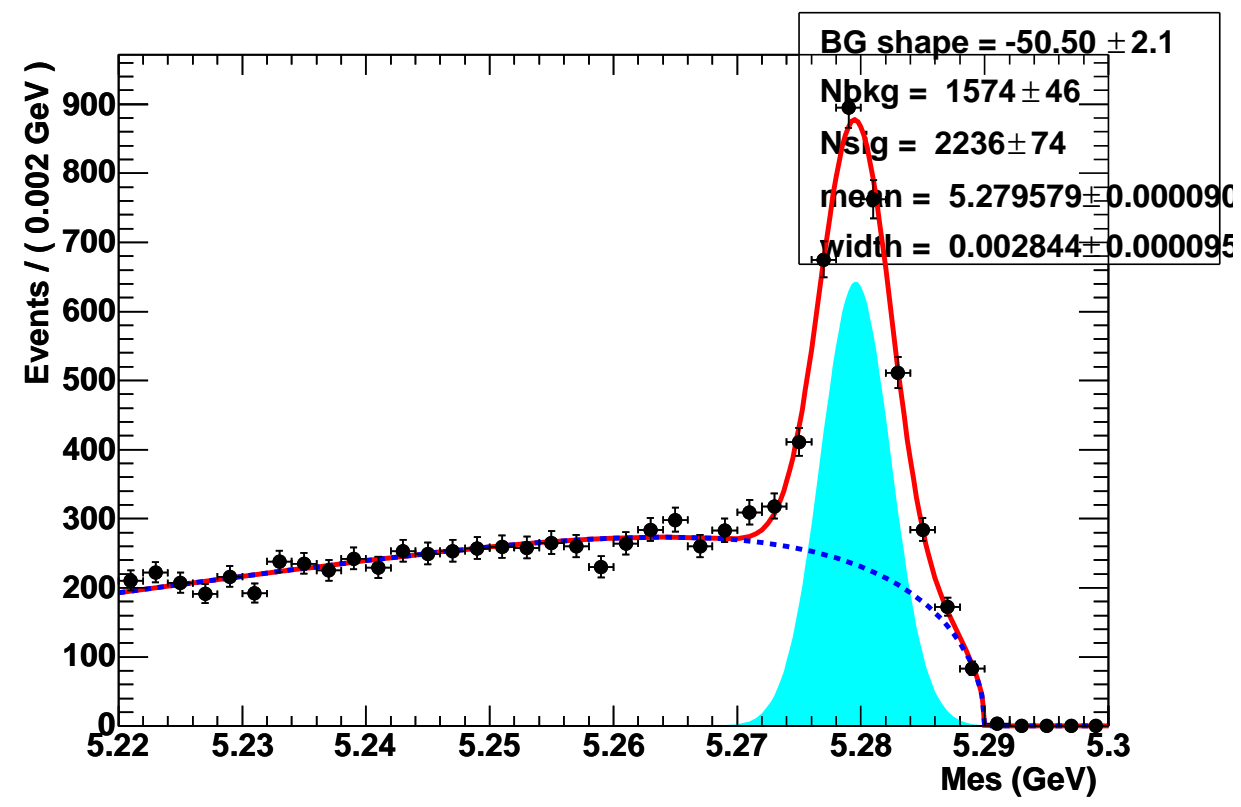

Figure 5.10: The $m_{\mathrm{ES}}$ distribution using $D^{0} \rightarrow K^{-} \pi^{+} \pi^{-} \pi^{+}$.

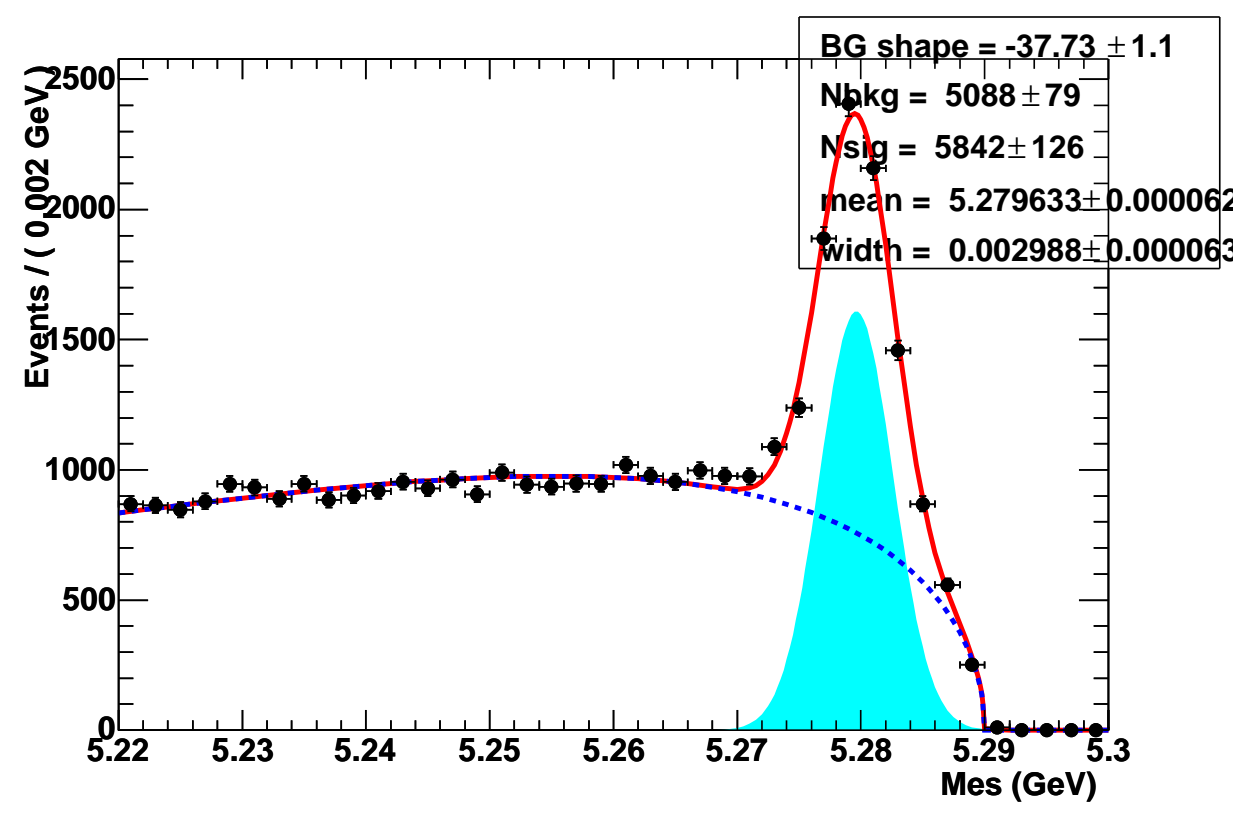

Figure 5.11: The $m_{\mathrm{ES}}$ distribution using $D^{+} \rightarrow K^{-} \pi^{+} \pi^{+}$. 


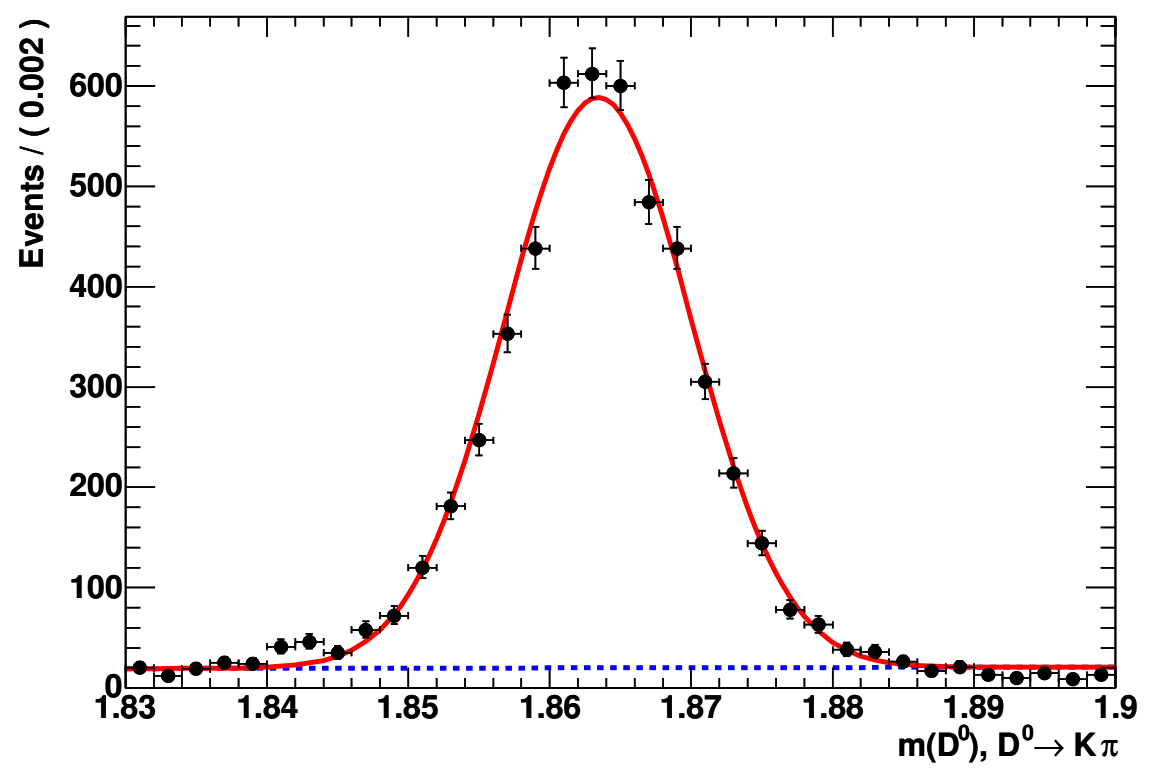

Figure 5.12: The $\Delta E$ distribution using $D^{0} \rightarrow K^{-} \pi^{+}$.

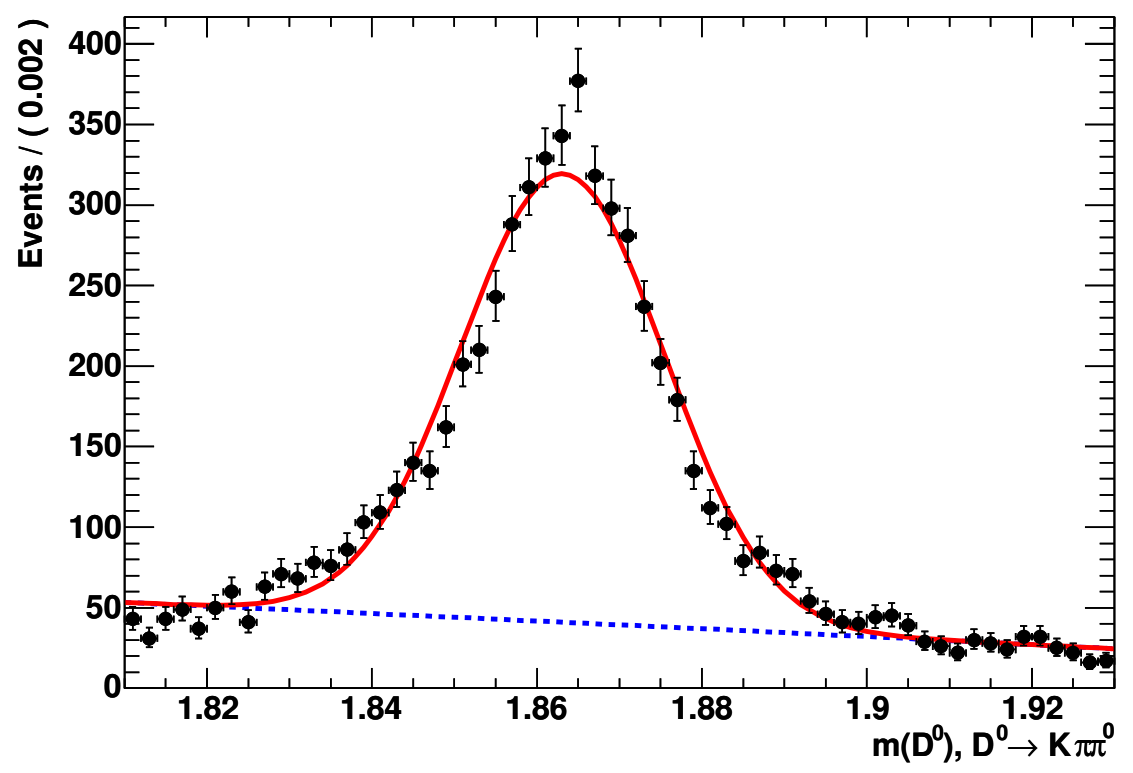

Figure 5.13: The $\Delta E$ distribution using $D^{0} \rightarrow K^{-} \pi^{+} \pi^{0}$. 


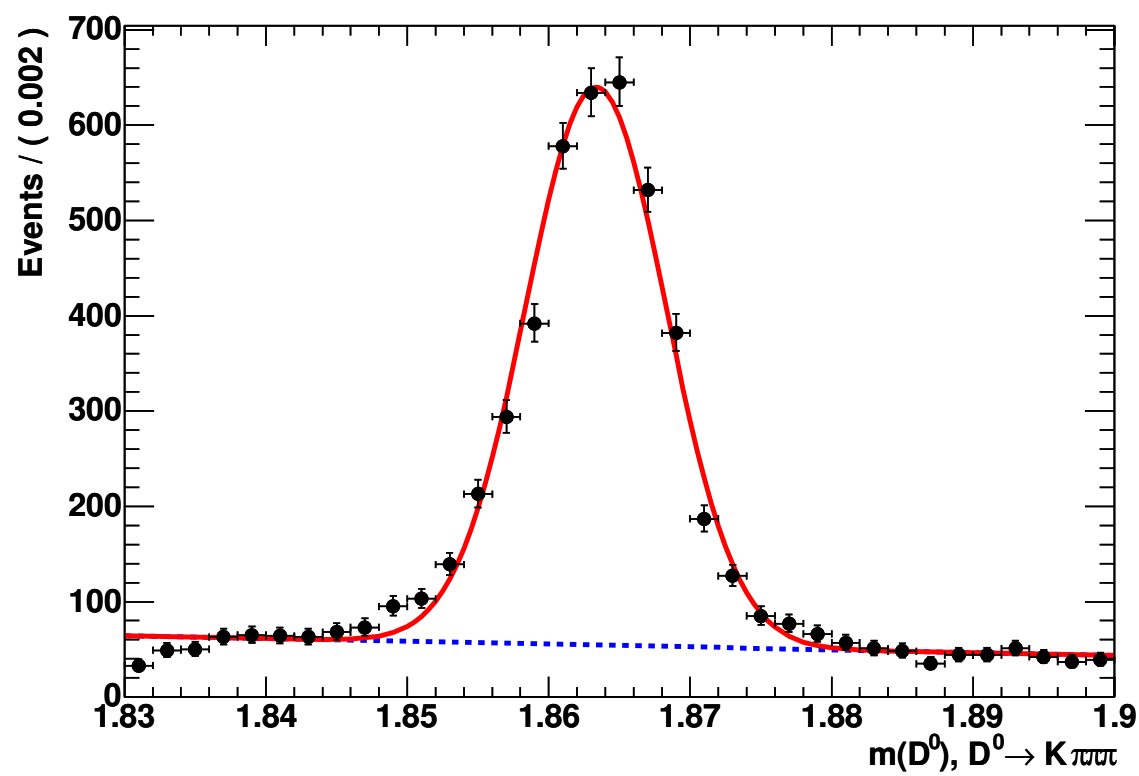

Figure 5.14: The $\Delta E$ distribution using $D^{0} \rightarrow K^{-} \pi^{+} \pi^{-} \pi^{+}$.

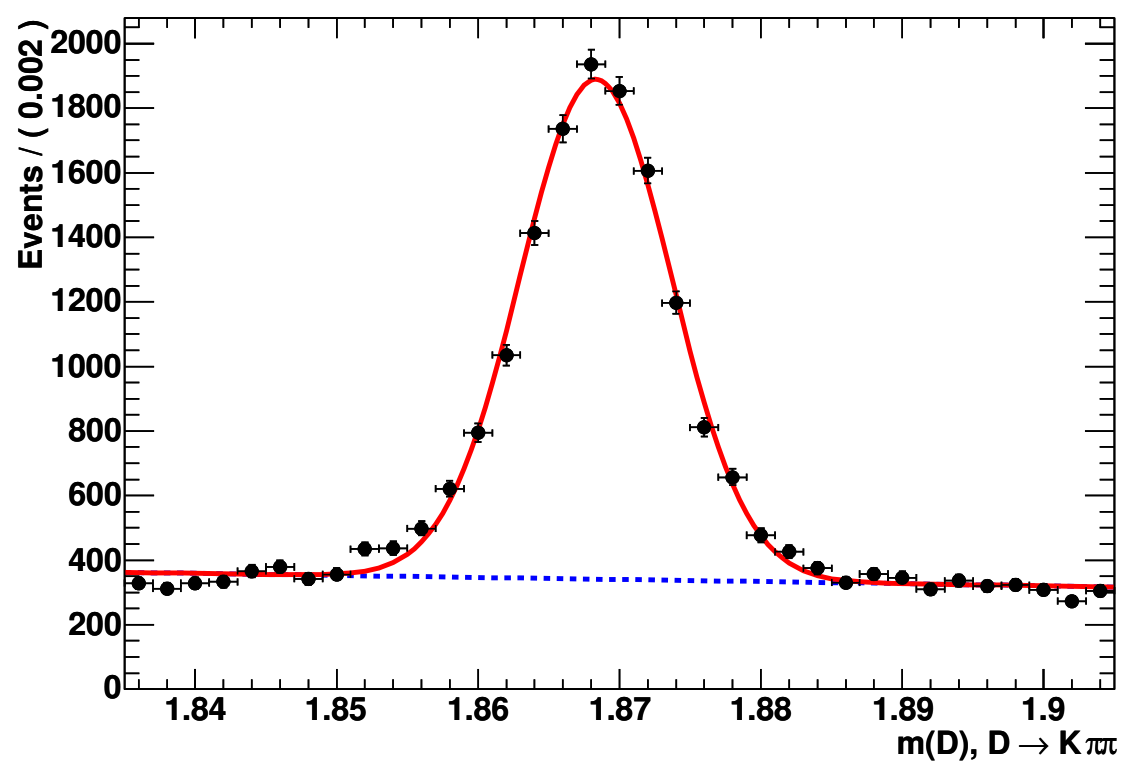

Figure 5.15: The $\Delta E$ distribution using $D^{+} \rightarrow K^{-} \pi^{+} \pi^{+}$. 


\subsubsection{Efficiencies}

The yields fitted from $\Delta E$ distributions in the signal MC are used to measure the reconstruction efficiencies as listed in Table 5.3. Combining these efficiencies together is done by weighted average using the branching fractions reported by BELLE (Table 5.5). Uncertainties on BELLE's measurements are included in the systematic error contribution but is less than $1 \%$.

\begin{tabular}{|l|c|c|c|c|c|c|}
\hline & \multicolumn{6}{|c|}{ Reconstruction efficiency (\%) } \\
\cline { 2 - 7 } & $D_{1}^{0}(2420)$ & $D_{2}^{* 0}(2460)$ & $D_{1}^{0}(j=1 / 2)$ & $D_{0}^{* 0}(j=1 / 2)$ & Non-Res. & Combined \\
\hline \hline$D^{0}$ decay mode & \multicolumn{7}{|c|}{$B^{-} \rightarrow D^{*+} \pi^{-} \pi^{-}$} \\
\hline$D^{0} \rightarrow K^{-} \pi^{+}$ & 21.3 & 22.04 & 21.03 & - & 21.57 & 21.30 \\
$D^{0} \rightarrow K^{-} \pi^{+} \pi^{0}$ & 5.79 & 5.74 & 5.68 & - & 5.77 & 5.74 \\
$D^{0} \rightarrow K^{-} \pi^{+} \pi^{-} \pi^{+}$ & 7.43 & 7.77 & 7.41 & - & 7.64 & 7.47 \\
\hline$D^{+}$decay mode & \multicolumn{7}{|c|}{$B^{-} \rightarrow D^{+} \pi^{-} \pi^{-}$} \\
\hline$D^{+} \rightarrow K^{-} \pi^{+} \pi^{+}$ & - & 16.90 & - & 16.48 & 17.26 & 16.67 \\
\hline
\end{tabular}

Table 5.3: The $\Delta E$ reconstruction efficiencies for signal Monte Carlo events in percentage.

\begin{tabular}{|l|c|}
\hline$D^{0} / D^{+}$Decay mode & Branching ratio (\%) \\
\hline$D^{0} \rightarrow K^{-} \pi^{+}$ & $3.80 \pm 0.07$ \\
$D^{0} \rightarrow K^{-} \pi^{+} \pi^{0}$ & $14.1 \pm 0.5$ \\
$D^{0} \rightarrow K^{-} \pi^{+} \pi^{-} \pi^{+}$ & $7.72 \pm 0.28$ \\
\hline$D^{+} \rightarrow K^{-} \pi^{+} \pi^{+}$ & $9.51 \pm 0.34$ \\
\hline
\end{tabular}

Table 5.4: The $D^{0}$ and $D^{+}$branching fractions of $D^{0}$ and $D^{+}$as given in the PDG [80]. 


\begin{tabular}{|c|c|}
\hline Mode & Branching fraction $\left(\times 10^{-3}\right)$ \\
\hline$B^{-} \rightarrow D_{1}(2420)^{0} \pi^{-}$ & 0.068 \\
$B^{-} \rightarrow D_{2}^{*}(2460)^{0} \pi^{-}\left(D_{2}^{*}(2460)^{0} \rightarrow D^{*} \pi\right)$ & 0.18 \\
$B^{-} \rightarrow D_{1}\left(j=\frac{1}{2}\right)^{0} \pi^{-}$ & 0.05 \\
$B^{-} \rightarrow D^{+*} \pi^{-} \pi^{-}(n r)$ & 1.25 \\
$B^{-} \rightarrow D_{2}^{*}(2460)^{0} \pi^{-}\left(D_{2}^{*}(2460)^{0} \rightarrow D \pi\right)$ & 0.034 \\
$B^{-} \rightarrow D_{0}^{*}\left(j=\frac{1}{2}\right)^{0} \pi^{-}$ & 0.061 \\
$B^{-} \rightarrow D^{+} \pi^{-} \pi^{-}(n r)$ & 1.02 \\
\hline
\end{tabular}

Table 5.5: BELLE numbers used for efficiency estimation [12].

\subsubsection{Peaking Background in the $\Delta E$ Distribution}

Peaking backgrounds (backgrounds which tend to peak in the signal region) arise from various causes. An example is the reconstruction of a $B^{-}$ candidate is created using a soft pion from the opposite $B^{+}$. Therefore the peaking backgrounds must be determined. This is measured using MC data. There are two sources used. The first is signal removed Generic MC data. Events in which the $B^{-}$decays to the $D^{*+} \pi^{-} \pi^{-}$and $D^{+} \pi^{-} \pi^{-}$final states from the selected $B^{-} \rightarrow D^{*+} \pi^{-} \pi^{-}$and $B^{-} \rightarrow D^{+} \pi^{-} \pi^{-}$samples are removed. From the $B^{-} \rightarrow D^{*+} \pi^{-} \pi^{-}$selection:

1. $B^{-} \rightarrow D_{2}^{* 0} \pi^{-}$with $D_{2}^{* 0} \rightarrow D^{*+} \pi^{-}$

2. $B^{-} \rightarrow D_{1}^{0}(2420) \pi^{-}$with $D_{1}^{0}(2420) \rightarrow D^{*+} \pi^{-}$

3. $B^{-} \rightarrow D_{1}^{0}(j=1 / 2) \pi^{-}$with $D_{1}^{0}(j=1 / 2) \rightarrow D^{*+} \pi^{-}$

4. $B^{-} \rightarrow D^{*+} \pi^{-} \pi^{-}$(non-resonant) 
are removed.

From the $B^{-} \rightarrow D^{+} \pi^{-} \pi^{-}$selection:

1. $B^{-} \rightarrow D_{2}^{* 0} \pi^{-}$with $D_{2}^{* 0} \rightarrow D^{+} \pi^{-}$

2. $B^{-} \rightarrow D_{0}^{* 0}(j=1 / 2) \pi^{-}$with $D_{0}^{* 0}(j=1 / 2) \rightarrow D^{+} \pi^{-}$

3. $B^{-} \rightarrow D^{+} \pi^{-} \pi^{-}$(non-resonant)

are removed.

The $\Delta E$ distributions are fit with a Gaussian for extracting the signal events plus a linear function for the background estimation. The peaking yields are shown in Table 5.6. There are only small numbers of background events that are seen to be peaking in the region of the signal events. The peaking background measurements are used to properly correct the amount of signal in the data to obtain branching fraction measurements (Figures 5.16 - 5.21).

\subsubsection{Inclusive Results}

The $\Delta E$ distributions of the selected $B^{-} \rightarrow D^{(*)+} \pi^{-} \pi^{-}$candidates are fitted with a Gaussian for the signal plus a linear function for the background. The results are shown in Table 5.7. The yields are determined from the fits to 


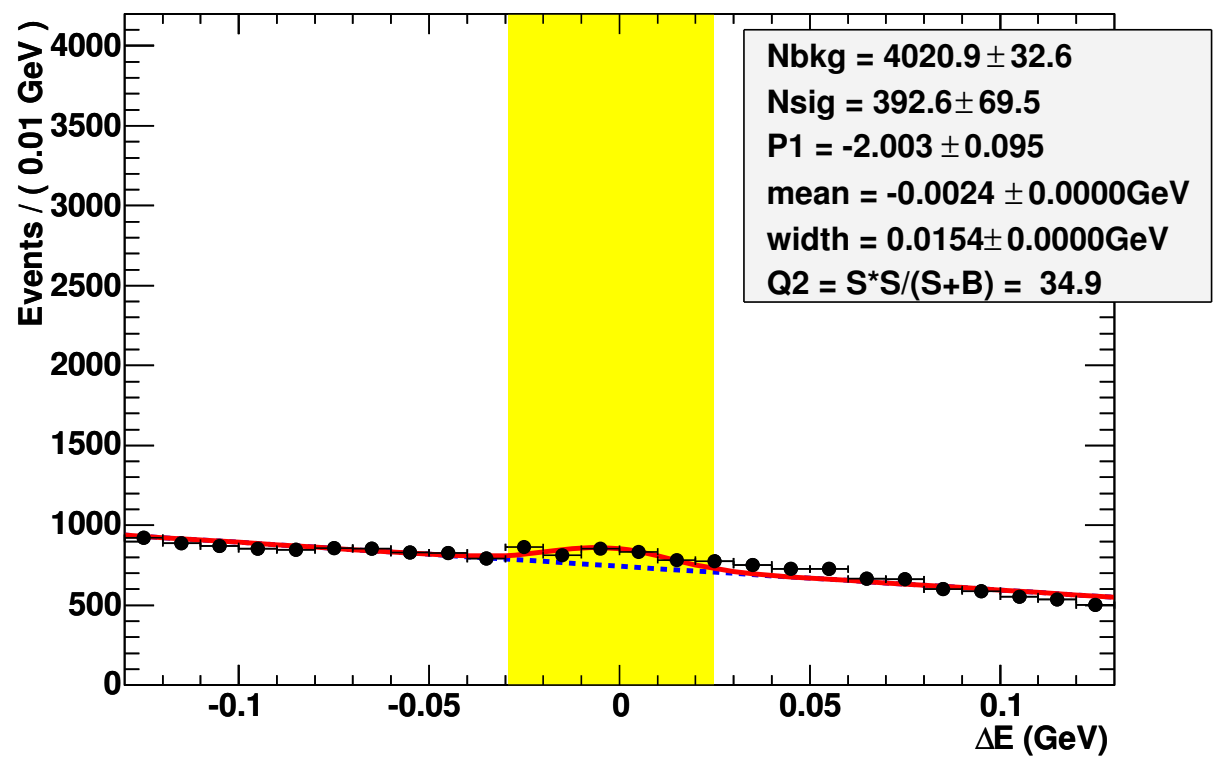

Figure 5.16: The $\Delta E$ distribution in the signal subtracted $B \bar{B}$ MC using the decays of $B^{-} \rightarrow D^{*+} \pi^{-} \pi^{-}\left(D^{0} \rightarrow K^{-} \pi^{+}, D^{0} \rightarrow K^{-} \pi^{+} \pi^{0}\right.$ and $\left.D^{0} \rightarrow K^{-} \pi^{+} \pi^{-} \pi^{+}\right)$. The yield for the peaking background in the MC sample is the area between the red and the dashed blue curve.

the $\Delta E$ distributions for the inclusive branching fraction measurements after subtracting the peaking component described in Section 5.4.2.

\section{$B^{-} \rightarrow D^{*+} \pi^{-} \pi^{-}$Results}

The inclusive $B^{-} \rightarrow D^{*+} \pi^{-} \pi^{-}$branching ratio for each $D^{0}$ decay mode $\left(D^{0} \rightarrow K^{-} \pi^{+}, D^{0} \rightarrow K^{-} \pi^{+} \pi^{0}, D^{0} \rightarrow K^{-} \pi^{+} \pi^{-} \pi^{+}\right)$is given by Equation 4.27, where the signal yields $Y_{\text {signal }, i}$ are given in Table 5.7. The $D^{0}$ branching fractions $\mathcal{B}\left(D^{0}\right)$ are given in Table 5.4. The $B^{-} \rightarrow D^{(*)+} \pi^{-} \pi^{-}$reconstruction efficiencies, $\epsilon_{i}$, are given in Table 5.3. The $D^{*+} \rightarrow D^{0} \pi^{+}$branching fraction 


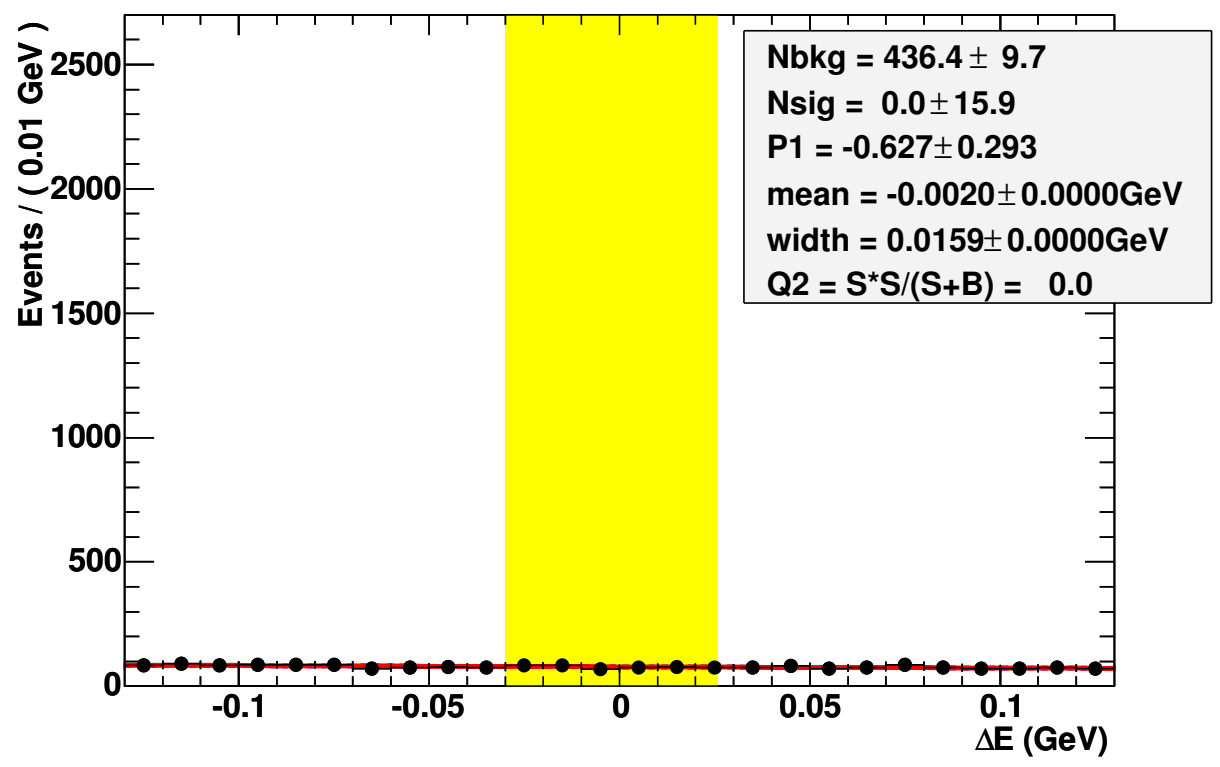

Figure 5.17: The $\Delta E$ distribution in the signal subtracted $q \bar{q} \mathrm{MC}$ using the decays of $B^{-} \rightarrow D^{*+} \pi^{-} \pi^{-}\left(D^{0} \rightarrow K^{-} \pi^{+}, D^{0} \rightarrow K^{-} \pi^{+} \pi^{0}\right.$ and $\left.D^{0} \rightarrow K^{-} \pi^{+} \pi^{-} \pi^{+}\right)$. The yield for the peaking background in the MC sample is the area between the red and the dashed blue curve.

comes from PDG, $\mathcal{B}\left(D^{*+}\right)=(67.7 \pm 0.5) \%$. The measured inclusive branching ratio's for each $D^{0}$ decay mode are given in Table 5.8 .

The signal region of $D^{*+} \pi^{-} \pi^{-}$final state expects to contain the following contributions to $B^{+}$decay:

1. $B^{-} \rightarrow D^{*+} \pi^{-} \pi^{-}$non-resonant decay,

2. $B^{-} \rightarrow D_{1}^{0}(2420) \pi^{-}$, where $D_{1}^{0}(2420) \rightarrow D^{*+} \pi^{-}$,

3. $B^{-} \rightarrow D_{2}^{* 0}(2460) \pi^{-}$, where $D_{2}^{* 0}(2460) \rightarrow D^{*+} \pi^{-}$, and

4. $B^{-} \rightarrow D_{1}^{0}(j=1 / 2) \pi^{-}$, where $D_{1}^{0}(j=1 / 2) \rightarrow D^{*+} \pi^{-}$. 


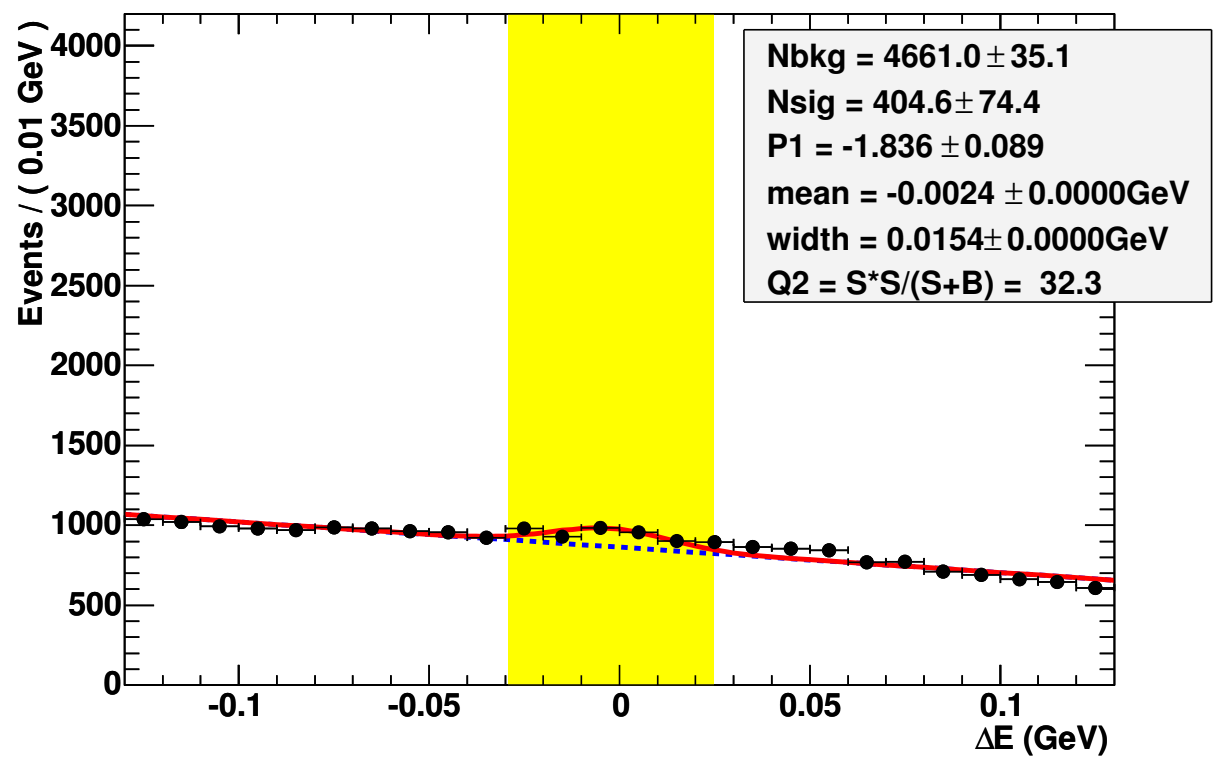

Figure 5.18: The $\Delta E$ distribution in the signal subtracted combined MC ( $q \bar{q}$ and $B \bar{B})$ using the decays of $B^{-} \rightarrow D^{*+} \pi^{-} \pi^{-}\left(D^{0} \rightarrow K^{-} \pi^{+}, D^{0} \rightarrow K^{-} \pi^{+} \pi^{0}\right.$ and $D^{0} \rightarrow K^{-} \pi^{+} \pi^{-} \pi^{+}$). The yield for the peaking background in the MC sample is the area between the red and the dashed blue curve.

The $B^{-} \rightarrow D^{*+} \pi^{-} \pi^{-}$branching ratio is measured by using the weighted mean of the branching fractions for the $D^{0}$ constructor modes.

The branching fraction measurement for the inclusive $B^{-} \rightarrow D^{*+} \pi^{-} \pi^{-}$ decay is:

$$
\mathcal{B}\left(B^{-} \rightarrow D^{*+} \pi^{-} \pi^{-}\right)=(1.67 \pm 0.03(\text { stat. })) \times 10^{-3}
$$




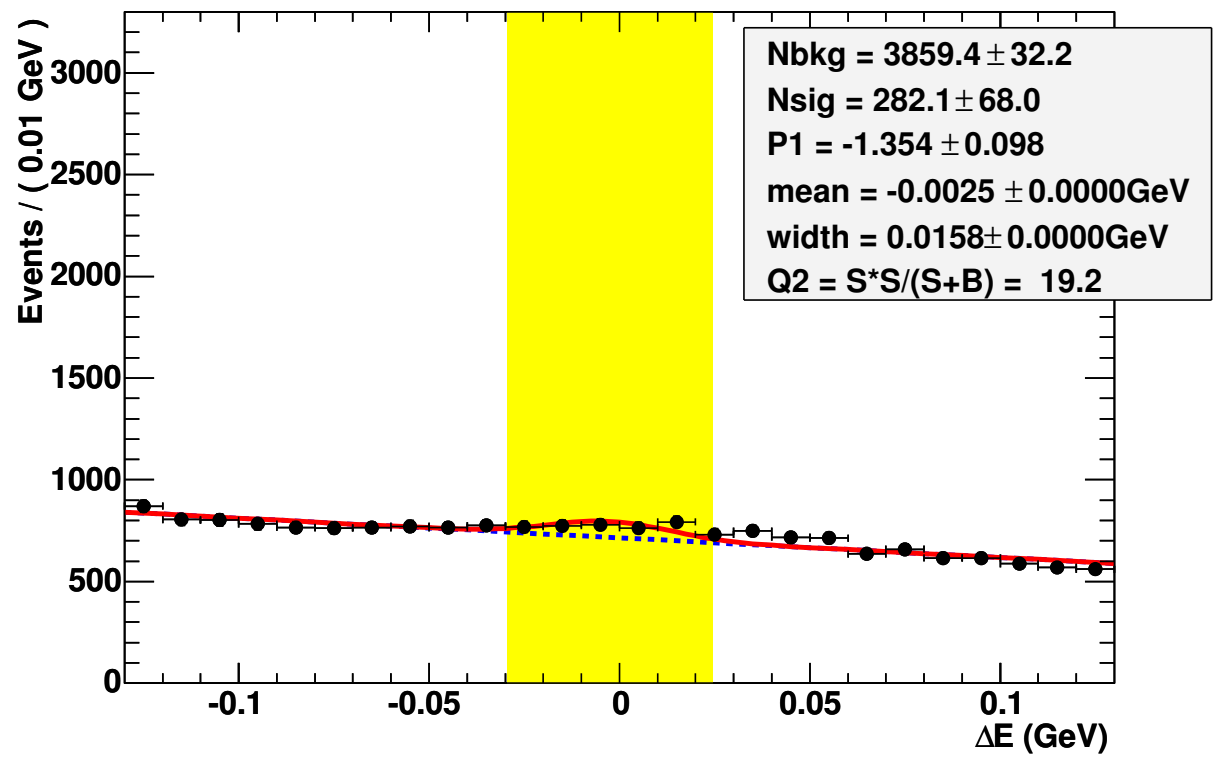

Figure 5.19: The $\Delta E$ distribution in the signal subtracted $B \bar{B} \mathrm{MC}$ using the decays of $B^{-} \rightarrow D^{+} \pi^{-} \pi^{-}\left(D^{+} \rightarrow K^{-} \pi^{+} \pi^{+}\right)$. The yield for the peaking background in the MC sample is the area between the red and the dashed blue curve.

$\mathcal{B}\left(B^{-} \rightarrow D^{+} \pi^{-} \pi^{-}\right)$Results

The inclusive $\mathcal{B}\left(B^{-} \rightarrow D^{+} \pi^{-} \pi^{-}\right)$branching ratio is given by Equation 4.24 , where the fitted yields $Y_{\text {signal }}$ are given in Table 5.7 , the $\mathcal{B}\left(B^{-} \rightarrow\right.$ $D^{+} \pi^{-} \pi^{-}$) reconstruction efficiency, $\epsilon$, is given in Table 5.3, and the total number of $B^{+}$events, $N_{B^{+} B^{-}}$.

The measured inclusive branching fraction is

$$
\mathcal{B}\left(B^{-} \rightarrow D^{+} \pi^{-} \pi^{-}\right)=(1.12 \pm 0.02(\text { stat. })) \times 10^{-3}
$$




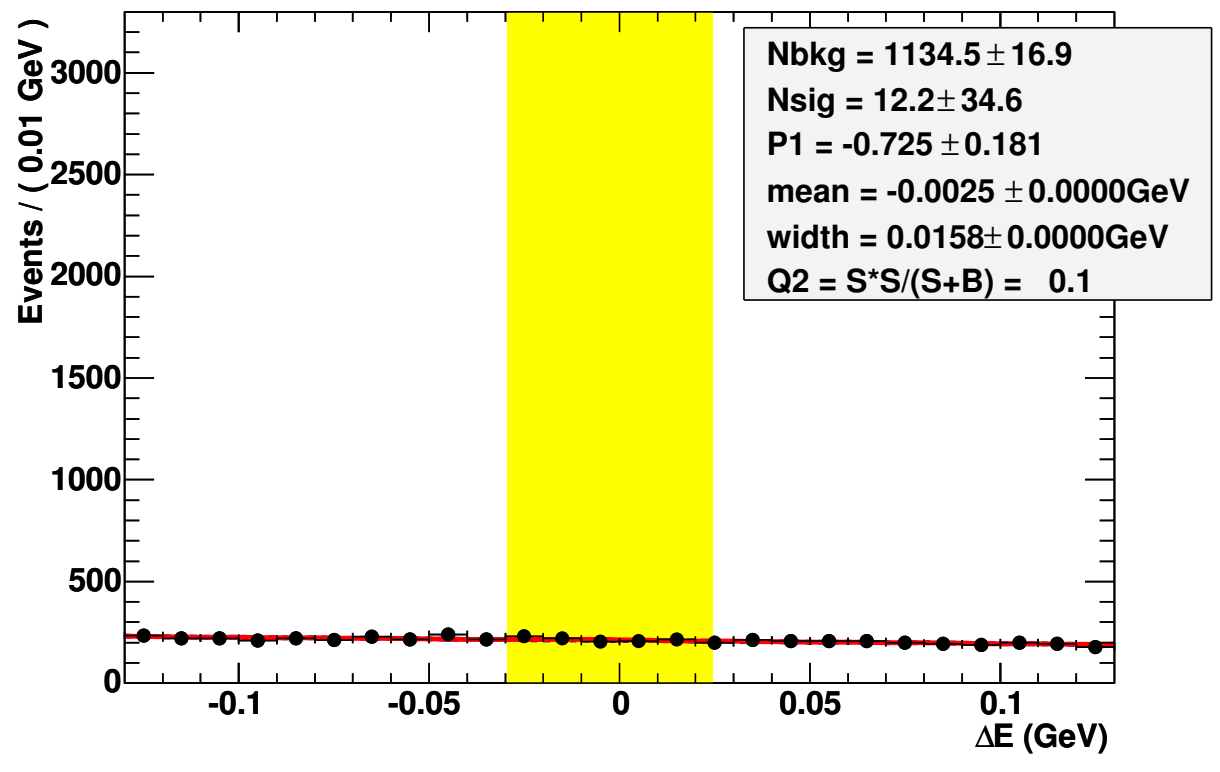

Figure 5.20: The $\Delta E$ distribution in the signal subtracted $q \bar{q} \mathrm{MC}$ using the decays of $B^{-} \rightarrow D^{+} \pi^{-} \pi^{-}\left(D^{+} \rightarrow K^{-} \pi^{+} \pi^{+}\right)$. The yield for the peaking background in the MC sample is the area between the red and the dashed blue curve.

The signal region of $D^{+} \pi^{-} \pi^{-}$final state is expected to contain the following $B^{-}$decays:

1. $B^{-} \rightarrow D^{+} \pi^{-} \pi^{-}$non-resonant decay,

2. $B^{-} \rightarrow D_{2}^{* 0}(2460) \pi^{-}$, where $D_{2}^{* 0}(2460) \rightarrow D^{+} \pi^{-}$, and

3. $B^{-} \rightarrow D_{0}^{* 0}(j=1 / 2) \pi^{-}$, where $D_{0}^{* 0}(j=1 / 2) \rightarrow D^{+} \pi^{-}$. 


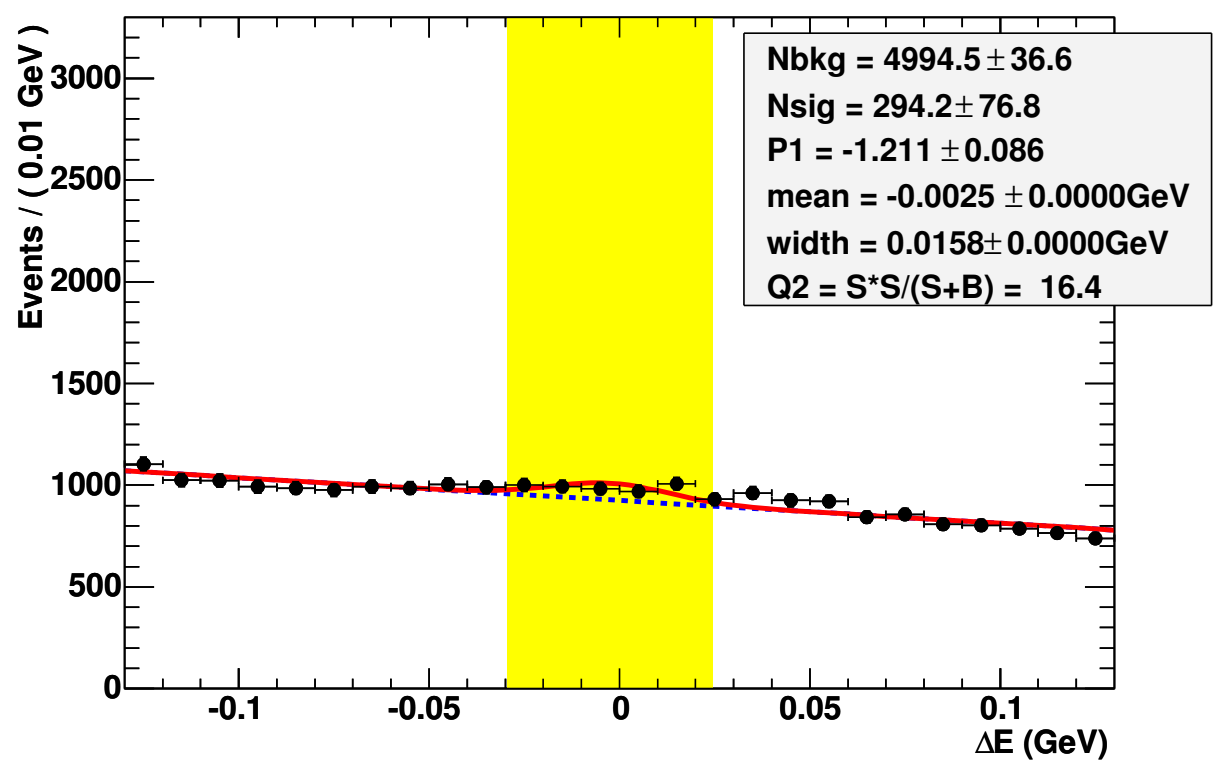

Figure 5.21: The $\Delta E$ distribution in the signal subtracted combined MC ( $q \bar{q}$ and $B \bar{B})$ using the decays of $B^{-} \rightarrow D^{+} \pi^{-} \pi^{-}\left(D^{+} \rightarrow K^{-} \pi^{+} \pi^{+}\right)$. The yield for the peaking background in the $\mathrm{MC}$ sample is the area between the red and the dashed blue curve.

\begin{tabular}{|l|c|c|c|}
\hline \multicolumn{4}{|c|}{$B^{-} \rightarrow D^{*+} \pi^{-} \pi^{-}$} \\
\hline Peaking background & generic $q \bar{q}$ & generic $B \bar{B}$ & Combined \\
\hline$D^{0} \rightarrow K^{-} \pi^{+}$ & $4 \pm 16$ & $141 \pm 40$ & $145 \pm 43$ \\
$D^{0} \rightarrow K^{-} \pi^{+} \pi^{0}$ & $18 \pm 17$ & $124 \pm 43$ & $142 \pm 46$ \\
$D^{0} \rightarrow K^{-} \pi^{+} \pi^{-} \pi^{+}$ & $0 \pm 12$ & $129 \pm 37$ & $121 \pm 39$ \\
\hline \multicolumn{4}{|c}{$B^{-} \rightarrow D^{+} \pi^{-} \pi^{-}$} \\
\hline$D^{+} \rightarrow K^{-} \pi^{+} \pi^{+}$ & $12 \pm 35$ & $281 \pm 68$ & $294 \pm 77$ \\
\hline
\end{tabular}

Table 5.6: Estimates of peaking backgrounds from generic $B \bar{B}$ events to the fitted $\Delta E$ signal peaks for the selected $B^{-} \rightarrow D^{*+} \pi^{-} \pi^{-}$and $B^{-} \rightarrow D^{+} \pi^{-} \pi^{-}$samples. 


\begin{tabular}{|l|r|r|c|}
\hline Decay Mode & $\Delta E(\mathrm{MeV})$ & Width $(\mathrm{MeV})$ & $N_{\text {Signal }}$ \\
\hline \multicolumn{4}{|c|}{$B^{-} \rightarrow D^{*+} \pi^{-} \pi^{-}$} \\
\hline$D^{0} \rightarrow K^{-} \pi^{+}$ & $-2.2 \pm 0.4$ & $15.7 \pm 0.4$ & $3777 \pm 75$ \\
$D^{0} \rightarrow K^{-} \pi^{+} \pi^{0}$ & $-2.5 \pm 0.4$ & $16.5 \pm 0.5$ & $3348 \pm 78$ \\
$D^{0} \rightarrow K^{-} \pi^{+} \pi^{-} \pi^{+}$ & $-2.4 \pm 0.4$ & $13.8 \pm 0.4$ & $2787 \pm 71$ \\
\hline \multicolumn{4}{|c|}{$B^{-} \rightarrow D^{+} \pi^{-} \pi^{-}$} \\
\hline$D^{+} \rightarrow K^{-} \pi^{+} \pi^{+}$ & $-2.5 \pm 0.3$ & $15.8 \pm 0.3$ & $7076 \pm 122$ \\
\hline
\end{tabular}

Table 5.7: The $\Delta E$ fit results: $\Delta E$ mean and resolution, number of signal candidates, $Q^{2}$ optimization ratio in the signal region.

\begin{tabular}{|l|c|}
\hline$D^{0}$ decay mode & $\mathcal{B}\left(B^{-} \rightarrow D^{*+} \pi^{-} \pi^{-}\left(D^{*+} \rightarrow D^{0} \pi^{+}\right)\right)\left(\times 10^{-3}\right)$ \\
\hline$D^{0} \rightarrow K^{-} \pi^{+}$ & $1.73 \pm 0.04$ (stat) \\
$D^{0} \rightarrow K^{-} \pi^{+} \pi^{0}$ & $1.53 \pm 0.04$ (stat) \\
$D^{0} \rightarrow K^{-} \pi^{+} \pi^{-} \pi^{+}$ & $1.78 \pm 0.05$ (stat) \\
\hline
\end{tabular}

Table 5.8: Inclusive branching ratios for $B^{-} \rightarrow D^{*+} \pi^{-} \pi^{-}$for each $D^{0}$ decay mode. The errors are statistical only. 


\subsubsection{Discussion and Comparison}

The $\mathcal{B}\left(B^{-} \rightarrow D^{+} \pi^{-} \pi^{-}\right)$inclusive measurement is very close to BELLE's result. The inclusive measurement for $\mathcal{B}\left(B^{-} \rightarrow D^{*+} \pi^{-} \pi^{-}\right)$, however, shows a significant departure from BELLE.

\begin{tabular}{|l|c|c|}
\hline & This Analysis & BELLE [12] \\
\hline & \multicolumn{2}{|c|}{ Branching Fraction $\left(10^{-3}\right)$} \\
\hline$B^{-} \rightarrow D^{*+} \pi^{-} \pi^{-}$ & $1.67 \pm 0.03 \pm 0.15$ & $1.25 \pm 0.08 \pm 0.22$ \\
$B^{-} \rightarrow D^{+} \pi^{-} \pi^{-}$ & $1.12 \pm 0.02 \pm 0.09$ & $1.02 \pm 0.04 \pm 0.15$ \\
\hline
\end{tabular}

Table 5.9: Comparison among the measurements.

Considerable effort was expended on consistency checks for these results. We tested the stability of the results using a variety of alternate analysis methods:

1. Alternate reconstruction methods: CompositionTools vs. SimpleComposition

2. Alternate vertexing methods: GenKin vs. TreeFitter

3. Alternate kaon ID selection: KMicroTight vs. KLHTight

4. Alternate tracks selection: GoodTracksVeryLoose vs. GoodTracksLoose

5. Alternate Monte Carlo generation: including or not including final state bremsstrahlung simulation by PHOTOS [38] 
6. Alternate best $B$-candidate selection: $m_{\mathrm{ES}}$ vs. chi-squared from vertexing.

The effect from removing the PHOTOS events is summarized in Table 5.10 .

\begin{tabular}{|l|c|}
\hline$D^{0} / D^{+}$Decay Mode & Branching Ratio $\left(10^{-3}\right)$ \\
\hline$D^{0} \rightarrow K^{-} \pi^{+}$ & $1.66 \pm 0.04$ \\
$D^{0} \rightarrow K^{-} \pi^{+} \pi^{0}$ & $1.47 \pm 0.04$ \\
$D^{0} \rightarrow K^{-} \pi^{+} \pi^{-} \pi^{+}$ & $1.72 \pm 0.05$ \\
\hline Combined & $1.61 \pm 0.03$ \\
\hline$D^{+} \rightarrow K^{-} \pi^{+} \pi^{+}$ & $1.07 \pm 0.02$ \\
\hline
\end{tabular}

Table 5.10: Calculated branching fractions after removing PHOTOS events in the signal MC. The errors are statistical only.

The results using the best $m_{\mathrm{ES}}$ value rather than using the best $\chi^{2}$ of the vertex fit did not show a significant difference and are shown in Table 5.11.

\begin{tabular}{|l|c|}
\hline$D^{0} / D^{+}$Decay Mode & Branching Ratio $\left(10^{-3}\right)$ \\
\hline$D^{0} \rightarrow K^{-} \pi^{+}$ & $1.71 \pm 0.02$ \\
$D^{0} \rightarrow K^{-} \pi^{+} \pi^{0}$ & $1.52 \pm 0.04$ \\
$D^{0} \rightarrow K^{-} \pi^{+} \pi^{-} \pi^{+}$ & $1.79 \pm 0.05$ \\
\hline Combined & $1.66 \pm 0.03$ \\
\hline$D^{+} \rightarrow K^{-} \pi^{+} \pi^{+}$ & $1.10 \pm 0.02$ \\
\hline
\end{tabular}

Table 5.11: Calculated branching fractions after applying $m_{\mathrm{ES}}$-based best candidate selection. The errors are statistical only.

The results were stable under all of these cross checks. 


\section{Reproducing simulation branching fractions}

The final sanity check was to repeat the analysis using Generic MC. The analysis reproduced the branching fraction values set by the MC AWG as seen in Table 5.12.

\begin{tabular}{|l|c|c|}
\hline \multirow{2}{*}{ mode } & \multicolumn{2}{|c|}{ Branching Fraction $\left(10^{-3}\right)$} \\
\cline { 2 - 3 } & Generated & Measured \\
\hline$D^{0} \rightarrow K^{-} \pi^{+}$ & & $2.60 \pm 0.04$ \\
$D^{0} \rightarrow K^{-} \pi^{+} \pi^{0}$ & & $2.51 \pm 0.05$ \\
$D^{0} \rightarrow K^{-} \pi^{+} \pi^{-} \pi^{+}$ & & $2.62 \pm 0.05$ \\
\hline Combined & 2.58 & $2.57 \pm 0.03$ \\
\hline \multicolumn{2}{|c}{$B^{-} \rightarrow D^{*+} \pi^{-} \pi^{-}$} \\
\hline & 1.99 & $1.99 \pm 0.03$ \\
\hline
\end{tabular}

Table 5.12: Branching fractions used in the generation of simulated sample are compared to those measured by using the analysis method.

\subsection{Results}

The inclusive branching fractions for $B^{-} \rightarrow D^{*+} \pi^{-} \pi^{-}$in the three decay modes of $D^{0}$ are

$$
\begin{aligned}
\mathcal{B}\left(B^{-} \rightarrow D^{*+} \pi^{-} \pi^{-}\left(D^{0} \rightarrow K^{-} \pi^{+}\right)\right) & =(1.73 \pm 0.04(\text { stat }) \pm 0.11(\text { syst })) \times 10^{-3} \\
\mathcal{B}\left(B^{-} \rightarrow D^{*+} \pi^{-} \pi^{-}\left(D^{0} \rightarrow K^{-} \pi^{+} \pi^{0}\right)\right) & =(1.53 \pm 0.04(\text { stat }) \pm 0.11(\text { syst })) \times 10^{-3} \\
\mathcal{B}\left(B^{-} \rightarrow D^{*+} \pi^{-} \pi^{-}\left(D^{0} \rightarrow K^{-} \pi^{+} \pi^{-} \pi^{+}\right)\right) & =(1.78 \pm 0.05(\text { stat }) \pm 0.15(\text { syst })) \times 10^{-3}
\end{aligned}
$$


The weighted average of these measurements yields:

$$
\mathcal{B}\left(B^{-} \rightarrow D^{*+} \pi^{-} \pi^{-}\right)=(1.67 \pm 0.03(\text { stat }) \pm 0.13(\text { syst })) \times 10^{-3}
$$

For $D^{+} \rightarrow K^{-} \pi^{+} \pi^{+}$, the inclusive branching fraction is:

$$
\mathcal{B}\left(B^{-} \rightarrow D^{+} \pi^{-} \pi^{-}\right)=(1.12 \pm 0.02(\text { stat }) \pm 0.08(\text { syst })) \times 10^{-3} .
$$

\subsection{Postscript: Hints of Future Directions}

Exclusive branching fractions for $B^{-} \rightarrow D_{J}^{0} \pi^{-}$and details of the resonant substructure for $B^{-} \rightarrow D^{*+} \pi^{-} \pi^{-}$and $B^{-} \rightarrow D^{+} \pi^{-} \pi^{-}$will be measured in a Dalitz analysis. This is currently underway in association with collaborators from Iowa State University and Manchester. Fits to the line shape for $B^{-} \rightarrow D^{*+} \pi^{-} \pi^{-}$and $B^{-} \rightarrow D^{+} \pi^{-} \pi^{-}$have been made and give a first look at the masses and widths for the narrow and wide resonances. These are more or less in keeping with previous measurements at BELLE and CLEO. Improved measurements of branching ratios, such as $D^{0} \rightarrow K^{-} \pi^{+}$[39] [40], are underway and should lower systematic errors. 


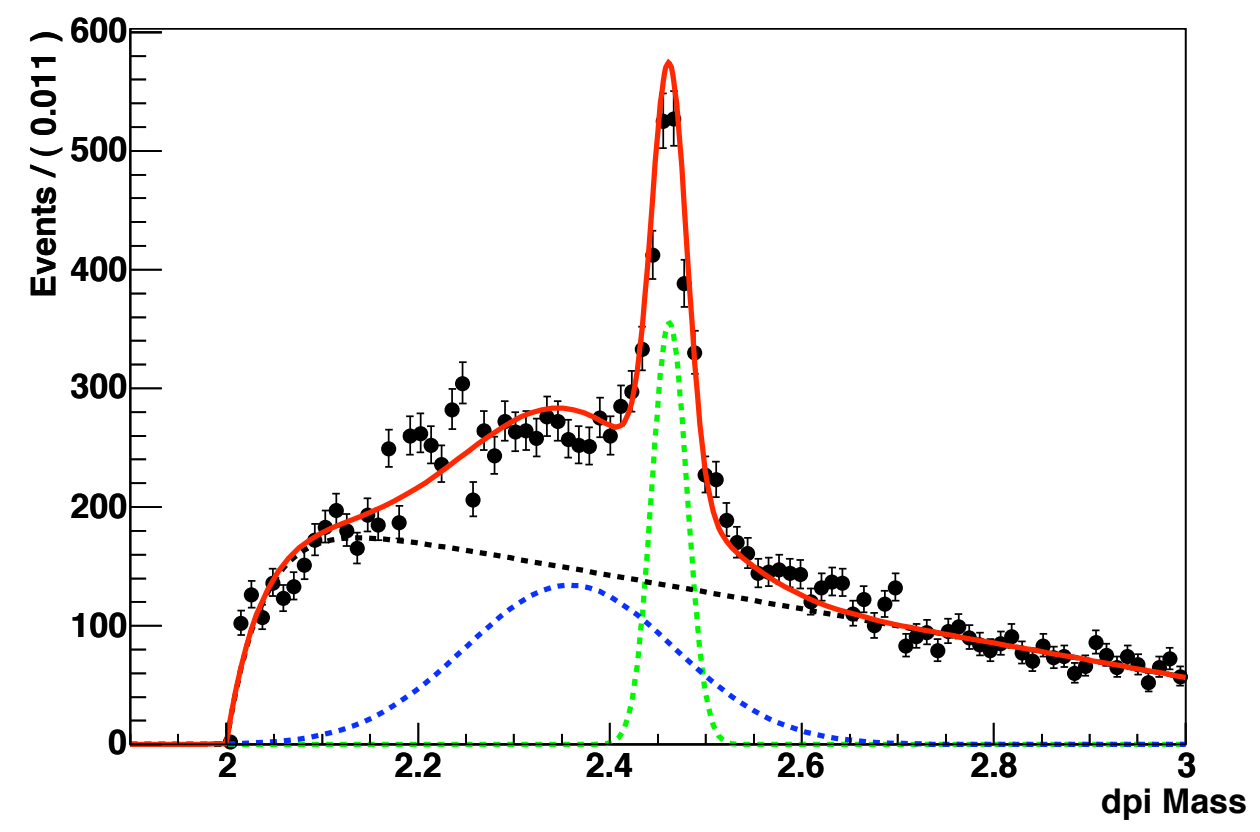

Figure 5.22: $m\left(D^{+} \pi^{-}\right)$spectrum.

$m\left(D^{+} \pi^{-}\right)$spectrum

Figure 5.22 shows the $m\left(D^{+} \pi^{-}\right)$spectrum. The background in the $\Delta E$ sideband as shown in Figure 5.23 is fitted and the result is used to determine the background shape for the signal region. The mass spectrum of Figure 5.22 was fitted with the following components:

1. $D_{2}^{*}(2460)^{0}$ resonance (green)

2. $D_{0}^{*}\left(j=\frac{1}{2}\right)^{0}$ resonance (blue)

3. Background as determined by the $\Delta E$ sideband (black). 


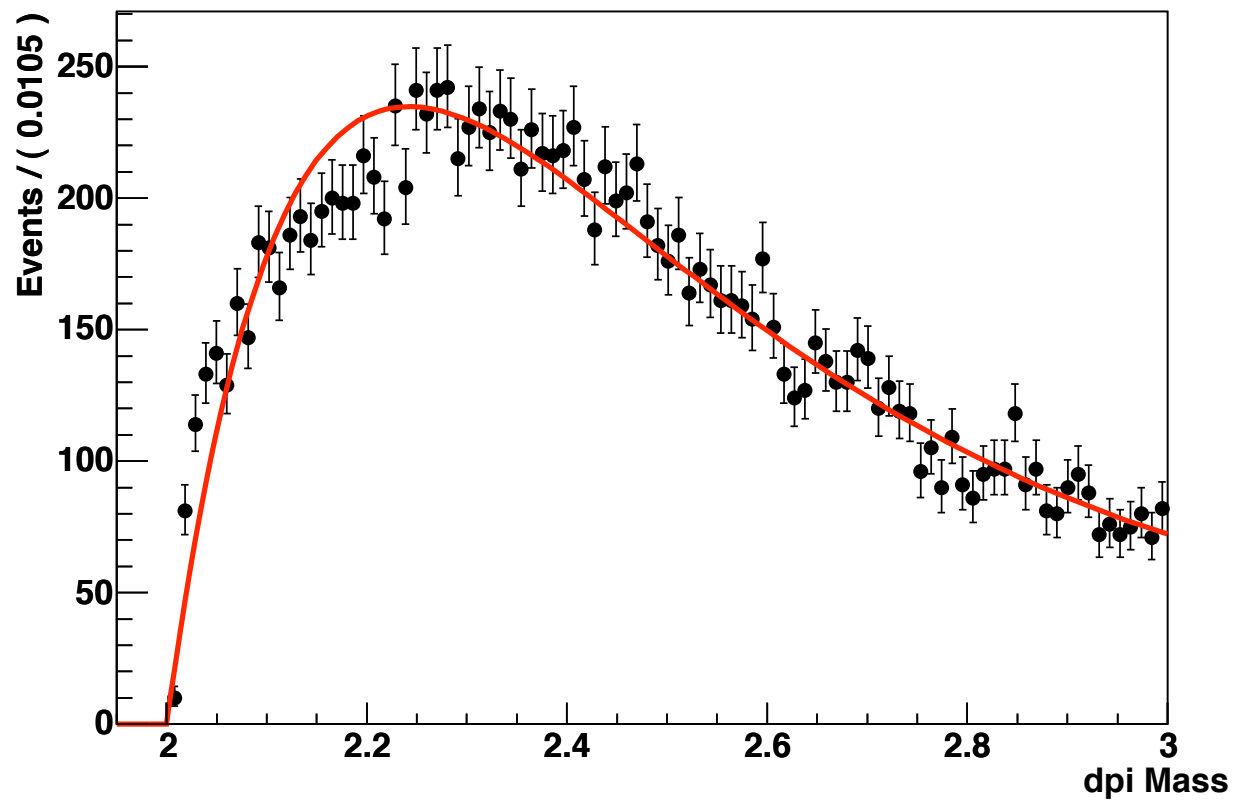

Figure 5.23: $m\left(D^{+} \pi^{-}\right)$spectrum, $\Delta E$ Sideband.

$m\left(D^{*+} \pi^{-}\right)$spectrum

A similar procedure is used for $m\left(D^{*+} \pi^{-}\right)$spectrum. The mass spectrum of Figure 5.25 was fitted with the following components:

1. $D_{2}^{*}(2460)^{0}$ resonance (green)

2. $D_{1}(2420)^{0}$ resonance (yellow)

3. $D_{1}\left(j=\frac{1}{2}\right)^{0}$ resonance (blue)

4. Background as determined by the $\Delta E$ sideband (black) of Figure 5.24. 


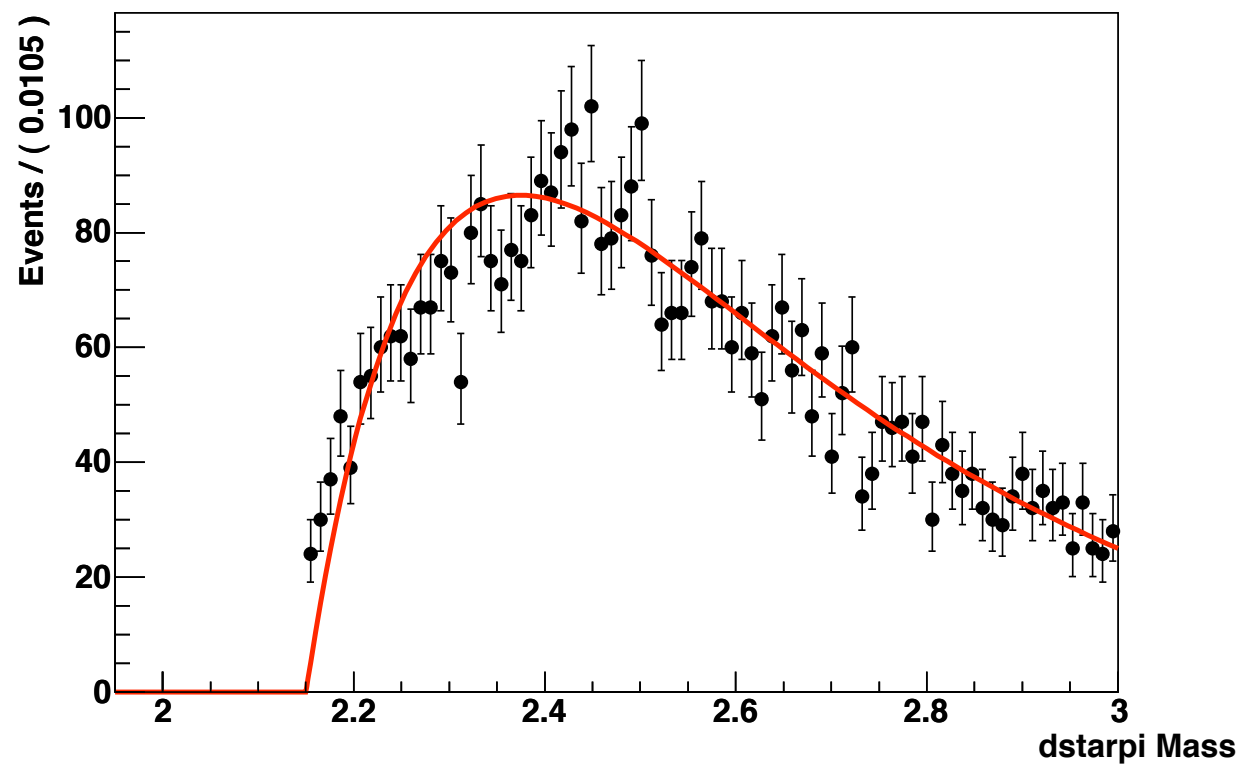

Figure 5.24: $m\left(D^{*+} \pi^{-}\right)$spectrum, $\Delta E$ Sideband.

\subsection{Closing}

This analysis made use of the data collected from Runs $1-5$ with total integrated luminosity of $343.38 \mathrm{fb}^{-1}$, which is corresponding to 383.92 million $B \bar{B}$ pairs, provided by the $B A B A R$ detector at the PEP-II asymmetric $B$ Factory. This dissertation presented the measurements of the inclusive branching fractions, $\mathcal{B}\left(B^{-} \rightarrow D^{*+} \pi^{-} \pi^{-}\right)$and $\mathcal{B}\left(B^{-} \rightarrow D^{+} \pi^{-} \pi^{-}\right)$:

$$
\begin{gathered}
\mathcal{B}\left(B^{-} \rightarrow D^{+} \pi^{-} \pi^{-}\right)=(1.12 \pm 0.02 \pm 0.08) \times 10^{-3} \\
\mathcal{B}\left(B^{-} \rightarrow D^{*+} \pi^{-} \pi^{-}\right)=(1.67 \pm 0.03 \pm 0.13) \times 10^{-3}
\end{gathered}
$$

$B A B A R$ 's high statistics, fine vertexing and tracking have provided for a very clean sample. The precision compares favorable to the previous best 


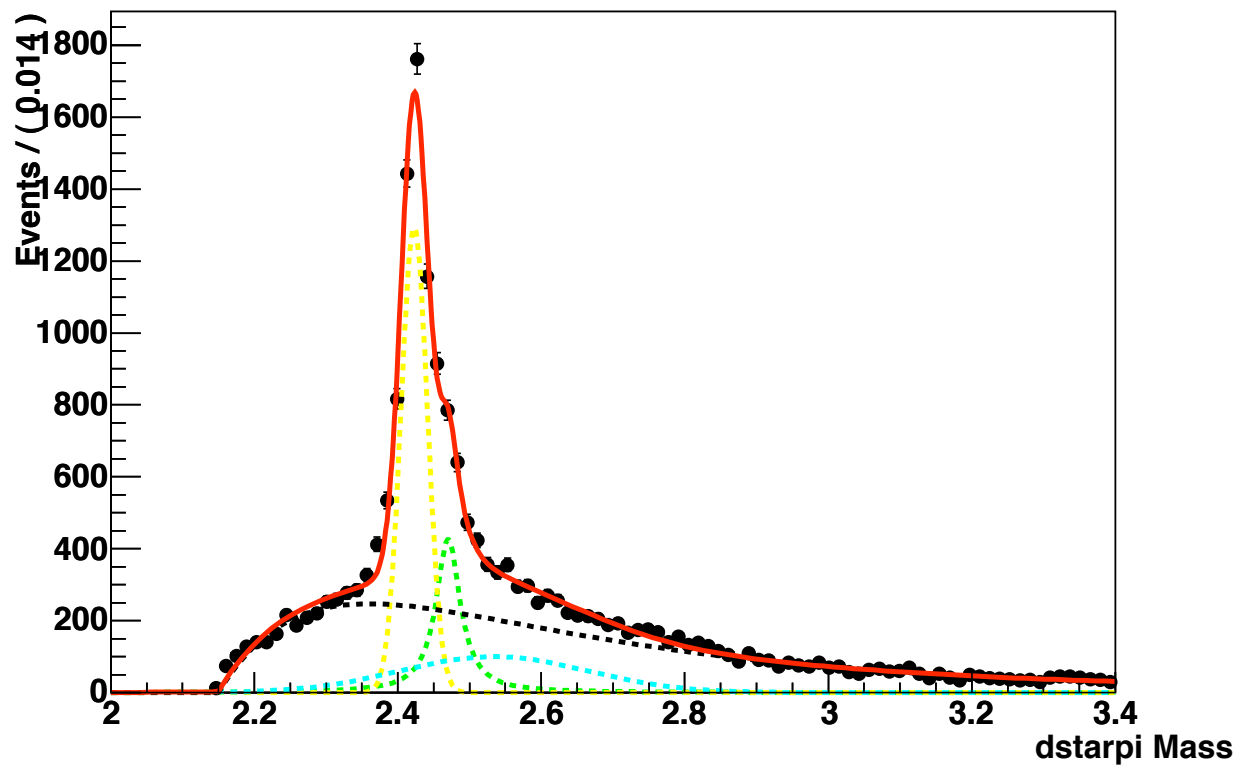

Figure 5.25: $m\left(D^{*+} \pi^{-}\right)$spectrum.

measurement of [12]:

$$
\begin{gathered}
\mathcal{B}\left(B^{-} \rightarrow D^{+} \pi^{-} \pi^{-}\right)=1.02 \pm 0.04 \pm 0.15 \\
\mathcal{B}\left(B^{-} \rightarrow D^{*+} \pi^{-} \pi^{-}\right)=1.25 \pm 0.07 \pm 0.22
\end{gathered}
$$

and the current world average of $B^{-} \rightarrow D^{*+} \pi^{-} \pi^{-}$[80]:

$$
\mathcal{B}\left(B^{-} \rightarrow D^{*+} \pi^{-} \pi^{-}\right)=1.35 \pm 0.22
$$


BIBLIOGRAPHY 


\section{Bibliography}

[1] Murray Gell-Mann, "A schematic model of baryons and mesons," Phys. Lett. 8 (1964) 214.

[2] V. E. Barnes et al., "Observation of a hyperon with strangeness -3," Phys. Rev. Lett. 12 (1964) 204.

[3] E288 Collaboration, S. W. Herb et al., "Observation of a dimuon resonance at $9.5-\mathrm{GeV}$ in $400-\mathrm{GeV}$ proton-nucleus collisions," Phys. Rev. Lett. 39 (1977) 252.

[4] ARGUS Collaboration, H. Albrecht et al., "Observation of the $D^{* 0}(2459)$ in $e^{+} e^{-}$annihilation," Phys. Lett. B221 (1989) 422.

[5] ARGUS Collaboration, H. Albrecht et al., "Resonance decomposition of the $\mathrm{D}^{*}(2420)^{0}$ through a decay angular analysis," Phys. Lett. B232 (1989) 398. 
[6] CLEO Collaboration, P. Avery et al., "P wave charmed mesons in $e^{+} e^{-}$ annihilation," Phys. Rev. D41 (1990) 774.

[7] CLEO Collaboration, P. Avery et al., "Production and decay of $\mathrm{D}_{1}(2420)^{0}$ and $\mathrm{D}_{2}^{*}(2460)^{0}, "$ Phys. Lett. B331 (1994) 236.

[8] E687 Collaboration, P. L. Frabetti et al., "Measurement of the masses and widths of $L=1$ charm mesons," Phys. Rev. Lett. 72 (1994) 324.

[9] CLEO Collaboration, S. Anderson et al., "Observation of a broad $L=1$ $c \bar{q}$ state in $B^{-} \rightarrow D^{*+} \pi^{-} \pi^{-}$at CLEO," Nucl. Phys. A663 (2000) 647.

[10] BABAR Collaboration, B. Aubert et al., "Study of the decays $B^{-} \rightarrow$ $D^{(*)+} \pi^{-} \pi^{-}, "$ LP03 - Batavia, hep-ex/0308026.

[11] FOCUS Collaboration, J. M. Link et al., "Measurement of masses and widths of excited charm mesons $D_{2}^{*}$ and evidence for broad states," Phys. Lett. B586 (2004) 11.

[12] Belle Collaboration, K. Abe et al., "Study of $B^{-} \rightarrow D^{* * 0} \pi^{-}\left(D^{* * 0} \rightarrow\right.$ $\left.D^{(*)+} \pi^{-}\right)$decays," Phys. Rev. D69 (2004) 112002.

[13] BABAR Collaboration, Vance O. Eschenburg, "Study of $B^{-} \rightarrow$ $D_{2}^{*}(2460)^{0} \pi^{-}$and $B^{-} \rightarrow D_{1}(2420)^{0} \pi^{-}$decays," Int. J. Mod. Phys. A20 (2005) 3698 . 
[14] CDF Collaboration, A. Abulencia et al., "Measurement of mass and width of the excited charmed meson states $D_{1}^{0}$ and $D_{2}^{* 0}$ at CDF," Phys. Rev. D73 (2006) 051104.

[15] Nathan Isgur and Mark B. Wise, "Weak decays of heavy mesons in the static quark approximation," Phys. Lett. B232 (1989) 113.

[16] Matthias Neubert, "Heavy-quark symmetry," Phys. Rept. 245 (1994) 259.

[17] Jean de Brunhoff, "Histoire de Babar, le petit éléphant," Jardin des Modes (Paris, 1931).

[18] ARGUS Collaboration, H. Albrecht et al., "Observation of a new charmed meson," Phys. Rev. Lett. 56 (1986) 549.

[19] E691 Collaboration, J. C. Anjos et al., "Observation of excited charmed mesons," Phys. Rev. Lett. 62 (1989) 1717.

[20] UA1 Collaboration, C. Albajar et al., "Search for $B^{0}-\bar{B}^{0}$ oscillations at the CERN proton-antiproton collider," Phys. Lett. B186 (1987) 247.

[21] P. Oddone, "Detector considerations," UCLA, eConf C870126 (1987) 423.

[22] "PEP-II: An Asymmetric B Factory, Conceptual Design Report," SLACR-418 (1993). 
[23] Michael S. Zisman, "High-intensity electron storage rings," Ann. Rev. Nucl. Part. Sci. 47 (1997) 315.

[24] BABAR Collaboration, B. Aubert et al., The BABAR detector," Nucl. Instrum. Meth. A479 (2002) 1.

[25] BABAR Collaboration, Patricia Burchat, "Development of a silicon vertex detector for an asymmetric B factory at SLAC," Nucl. Instrum. Meth. A342 (1994) 292.

[26] NA11 Collaboration, B. Hyams et al., "A silicon counter telescope to study shortlived particles in high-energy hadronic interactions," Nucl. Instrum. Meth. 205 (1983) 99.

[27] E691 Collaboration, P. E. Karchin et al., "Test beam studies of a silicon microstrip vertex detector," IEEE Trans. Nucl. Sci. 32 (1985) 612.

[28] E691 Collaboration, J. C. Anjos et al., "Measurement of the form-factors in the decay $D^{+} \rightarrow \bar{K}^{* 0} e^{+} \nu_{e}$," Phys. Rev. Lett. 65 (1990) 2630.

[29] BABAR Collaboration, Patricia R. Burchat, John Hiser, Adam Boyarski, and Don Briggs, "Studies of helium gas mixtures in drift chambers," Nucl. Instrum. Meth. A316 (1992) 217. 
[30] W. Zimmermann et al., "Helium-Propane as drift chamber gas," Nucl. Instrum. Meth. A243 (1986) 86.

[31] BABAR Collaboration, I. Adam et al., "The DIRC particle identification system for the BABAR experiment," Nucl. Instrum. Meth. A538 (2005) 281.

[32] BABAR Collaboration, R. Barlow et al., "Results from the BABAR electromagnetic calorimeter beam test," Nucl. Instrum. Meth. A420 (1999) 162.

[33] BABAR Collaboration, Johannes M. Bauer, "Absolute energy calibration with the neutron activated liquid source system at BABAR's CsI(Tl) calorimeter," IEEE Trans. Nucl. Sci. 51 (2004) 1596.

[34] BABAR Collaboration, Johannes M. Bauer, "The BABAR electromagnetic calorimeter: status and performance improvements," IEEE2005-Puerto Rico, physics/0601138.

[35] BABAR Collaboration, P. Fabbricatore et al., "The superconducting magnet for the BABAR detector of the PEP-II B factory at SLAC," IEEE Trans. Magnetics 32 (1996) 2210. 
[36] BABAR Collaboration, F. Anulli et al., "The BABAR instrumented flux return performance: lessons learned," Nucl. Instrum. Meth. A494 (2002) 455.

[37] BABAR Collaboration, F. Anulli et al., "BABAR forward endcap upgrade," Nucl. Instrum. Meth. A539 (2005) 155.

[38] E. Barberio and Z. Was, "PHOTOS: A universal Monte Carlo for QED radiative corrections, Version 2.0," Comput. Phys. Common. 79 (1994) 291.

[39] BABAR Collaboration B. Aubert et al., "Measurement of the absolute branching fraction, $D^{0} \rightarrow K^{-} \pi^{+}, "$ arXiv:0704.2080(2007).

[40] BABAR Collaboration, R. Godang, hep-ex/0612002.

[41] E791 Collaboration, Stephen B. Bracker et al., "A simple multiprocessor management system for event parallel computing," BABAR-Note-128, IEEE Trans. Nucl. Sci. 43 (1996) 2457.

[42] E791 Collaboration, E. M. Aitala et al., "Correlations between $D$ and $\bar{D}$ mesons produced in 500-GeV/c $\pi^{-}$nucleon interactions," Eur. Phys. J. direct C1 (1999) 4. 
[43] E791 Collaboration, E. M. Aitala et al., "Measurement of the form-factor ratios for $D^{+} \rightarrow \bar{K}^{* 0} \ell \nu_{\ell}$, " Phys. Lett. B440 (1998) 435 .

[44] BABAR Collaboration, Neil Geddes., "The BABAR computing model," Comput. Phys. Commun. 110 (1998) 38.

[45] D. A. Sanders et al., "Multi-terabyte EIDE disk arrays running Linux RAID5," CHEP '04-Interlaken, physics/0411188.

[46] B. Aubert et al. BABAR Collaboration, Phys. Rev.D 67, 032002 (2003).

[47] R. Barate et al., Phys. Lett. B 565, 61 (2003).

[48] Peter W. Higgs, "Broken symmetries, massless particles and gauge fields," Phys. Lett. 12 (1964) 132.

[49] Peter W. Higgs, "Broken symmetries and the masses of gauge bosons," Phys. Rev. Lett. 13 (1964) 508.

[50] Peter W. Higgs, "Broken symmetries and the masses of gauge bosons," Phys. Rev. 145 (1966) 1156.

[51] Steven Weinberg, "A model of leptons," Phys. Rev. Lett. 19 (1967) 1264.

[52] Sheldon L. Glashow, "Partial-symmetries of weak interactions," Nucl. Phys. 22 (1961) 579. 
[53] Abdus Salam and J. C. Ward, "Electromagnetic and weak interactions," Phys. Lett. 13 (1964) 168.

[54] Gargamelle Collaboration, F. J. Hasert et al., Observation of neutrino like interactions without muon or electron in the Gargamelle neutrino experiment," Phys. Lett. B46 (1973) 138.

[55] CPEPweb, Chart of Fundamental Particles and Interactions, 2005.

[56] UA1 Collaboration, G. Arnison et al., "Experimental observation of isolated large transverse energy electrons with associated missing energy at $\sqrt{s}=540-\mathrm{GeV}, "$ Phys. Lett. B122 (1983) 103.

[57] UA1 Collaboration, G. Arnison et al., "Experimental observation of lepton pairs of invariant mass around $95-\mathrm{GeV} / \mathrm{c}^{2}$ at the CERN SPS collider," Phys. Lett. B126 (1983) 398.

[58] UA2 Collaboration, M. Banner et al., "Observation of single isolated electrons of high transverse momentum in events with missing transverse energy at the CERN $\bar{p} p$ collider," Phys. Lett. B122 (1983) 476.

[59] UA2 Collaboration, P. Bagnia et al., "Evidence for $Z^{0} \rightarrow e^{+} e^{-}$at the CERN $\bar{p} p$ collider," Phys. Lett. B129 (1983) 130.

[60] BABAR Collaboration, BAD Note 116, (2001). 
[61] BABAR Collaboration, BAD Note 34, (2000).

[62] BABAR Collaboration, BAD Note 53, (2000).

[63] BABAR Collaboration, BAD Note 94, (2005).

[64] BABAR Collaboration, BAD Note 619, (2003).

[65] BABAR Collaboration, PID AWG,

http://www.slac.stanford.edu/BFROOT/www/Physics/

Tools/Pid/PidKilling/PidKilling.html

[66] BABAR Collaboration, Neutrals AWG,

http://www.slac.stanford.edu/BFROOT/www/Physics/

Analysis/AWG/Neutrals/validation/results/results.html

[67] T. E. Browder and R. Faccini, "Establishment of CP violation in B decays," Ann. Rev. Nucl. Part. Sci. 53 (2003) 353.

[68] CLEO Collaboration, C. Bebek et al., "Evidence for new flavor production at the $\Upsilon(4 S)$," Phys. Rev. Lett. 46 (1981) 84. 
[69] BABAR Collaboration, Romulus Godang, First measurement of the branching fraction of $e^{+} e^{-} \rightarrow B^{0} \bar{B}^{0}, "$ Int. J. Mod. Phys. A20 (2005) 3605.

[70] BABAR Collaboration, B. Aubert et al., "Measurement of the Branching Fraction $\Upsilon(4 S) \rightarrow \bar{B}$, Phys. Rev. Lett. 95, (1995) 042001.

[71] Heavy Flavor Averaging Group (HFAG), E. Barberio et al., hep-ex 0603003 (2006).

[72] BABAR Collaboration, B. Aubert et al., "Measurement of CP violating asymmetries in $B^{0}$ decays to CP eigenstates," Phys. Rev. Lett. 86 (2001) 2515.

[73] E-691 Collaboration, J.C. Aujos et al., Phys.Rev. D48 (1993) 56.

[74] Belle Collaboration, A. Abashian et al., "Measurement of the CP violation parameter $\sin 2 \phi_{1}$ in $B_{d}^{0}$ meson decays," Phys. Rev. Lett. 86 (2001) 2509.

[75] CDF Collaboration, T. Affolder et al., A Measurement of $\sin 2 \beta$ from $B \rightarrow J / \psi K_{S}^{0}$ with the CDF detector," Phys. Rev. D61 (2000) 072005.

[76] Stephen Godfrey and Richard Kokoski, "The properties of $\mathrm{P}$ wave mesons with one heavy quark," Phys. Rev. D43 (1991) 1679. 
[77] M. Neubert, "Theoretical analysis of $\bar{B} \rightarrow D^{* *} \pi$ decays," Phys. Lett. B418 (1998) 173.

[78] Adam F. Falk and Michael Luke, "Strong Decays of Excited Heavy Mesons in Chiral Perturbation Theory," hep-ph/9206241, (1992).

[79] Geoffrey C. Fox and Stephen Wolfram, "Observables for the analysis of event shapes in $e^{+} e^{-}$annihilation and other processes," Phys. Rev. Lett. $41(1978) 1581$.

[80] W. M. Yao et al., "Review of particle physics," J. Phys. G33 (2006). 


\section{Vita}

Son of a Californian Coast Guard officer and a Southern Belle from Alabama, Vance Onno Eschenburg was born in Fairfield, California. He called many places his home during his early years: San Rafael, California; Virginia Beach, Virginia; Slidell, Louisiana; Mobile, Alabama; Nimitz Hill, Guam; and Panama City, Panama. He later graduated with a Bachelor of Arts degree in Physics from the University of California at Berkeley. During that time, he was an active member of the Alpha Chapter of the Alpha Kappa Lambda fraternity.

Vance returned to the South to study physics at the University of Mississippi. There, he became the student of Dr. Robert Kroeger and a member of the research group studying high-energy physics. He also actively taught labs and tutored students at many levels. He is currently spending his second tour of service at the Stanford Linear Accelerator Center in Menlo Park, California. 
Vances interests are many. During high school, we was co-captain of the Quiz Bowl team and studied classical piano. Currently, he tends to his bonsai trees and his familys web site and scuba dives with his brother whenever he is near the Gulf of Mexico.

Out of suffering have emerged the strongest souls;

the most massive characters are seared with scars.

Kahlil Gibran Lebanese born American philosophical

Essayist, Novelist and Poet. 1883-1931 